\title{
Bispyridinium Dienes, Histone Deacetylase Inhibitors With Selective Activities
}

Carlos Pérez-Balado, ${ }^{a}$ Angela Nebbioso, ${ }^{\mathrm{b}}$ Paula Rodríguez-Graña, ${ }^{a}$ Annunziata Minichiello, ${ }^{\mathrm{b}}$ Marco Miceli, ${ }^{\mathrm{b}}$ Lucia Altucci ${ }^{\mathrm{b}, \mathrm{c},}$ and Ángel R. de Lera ${ }^{a *}$

${ }^{a}$ Departamento de Química Orgánica, Universidade de Vigo, Lagoas-Marcosende, 36310 Vigo, Spain.

${ }^{\mathrm{b}}$ Dipartimento di Patologia Generale, Seconda Università degli Studi di Napoli, Vico L. de Crecchio 780138 Napoli; ${ }^{c}$ NOGEC, Naples Oncogenomic Center, CEINGE Biotecnologia Avanzata, Napoli, Italy. 
2-(11-Bromoundecyloxy)-tetrahydro-2H-pyran (4a). To a stirred solution of 11bromoundecanol $(10.0 \mathrm{~g}, 33.80 \mathrm{mmol})$ in dichloromethane $(100 \mathrm{~mL})$ was added pyridinum para-toluenesulfonate $(100 \mathrm{mg}, 0.39 \mathrm{mmol}, 1 \mathrm{~mol} \%)$ and dihydropyran $(5.45$ $\mathrm{mL}, 59.70 \mathrm{mmol}, 1.5$ equiv). The resulting colourless solution was stirred at $25^{\circ} \mathrm{C}$ for $14 \mathrm{~h}$. Then it was washed with a $2 \mathrm{M}$ aqueous $\mathrm{Na}_{2} \mathrm{CO}_{3}$ solution $(2 \times 50 \mathrm{~mL})$. The combined organic layers were dried over $\mathrm{Na}_{2} \mathrm{SO}_{4}$ and the solvents removed under reduced pressure. The residue was purified by flash chromatography (96:4, hexaneEtOAc) to afford $12.85 \mathrm{~g}$ (96\% yield) of the title compound as a colourless oil. ${ }^{1} \mathrm{H}$ NMR $\left(400 \mathrm{MHz}, \mathrm{CDCl}_{3}\right) \delta 4.58$ (broad s, $\left.1 \mathrm{H}\right), 3.9-3.8(\mathrm{~m}, 1 \mathrm{H}), 3.8-3.7(\mathrm{~m}, 1 \mathrm{H}), 3.5-3.4(\mathrm{~m}$, $1 \mathrm{H}), 3.41(\mathrm{t}, J=6.9 \mathrm{~Hz}, 2 \mathrm{H}), 3.4-3.3(\mathrm{~m}, 1 \mathrm{H}), 1.9-1.8(\mathrm{~m}, 3 \mathrm{H}), 1.71(\mathrm{t}, J=10.7 \mathrm{~Hz}$, 1H), 1.6-1.5 (m, 5H), 1.5-1.2 (m, 15H) ppm. $\left.{ }^{13} \mathrm{C} \mathrm{NMR} \mathrm{(100} \mathrm{MHz,} \mathrm{CDCl}_{3}\right) \delta$ 98.8, 67.6, $62.3,34.0,32.8,30.7,29.7,29.5,29.4,29.3,28.7,28.1,26.2,25.5,19.7$ ppm. IR $(\mathrm{NaCl})$ $v 2924(\mathrm{~s}, \mathrm{C}-\mathrm{H}), 2852$ (s, C-H), 1635 (w), 1459 (m), 1350 (m), 1258 (w), 1120 (s), 1075 (s), 1028 (s). MS (EI $\left.{ }^{+}\right) m / z(\%) 335$ ([M-1 $\left.]^{+}\left[{ }^{81} \mathrm{Br}\right], 2\right), 333$ ([M-1] $\left.{ }^{+}\left[{ }^{79} \mathrm{Br}\right], 2\right), 164$ (33), 162 (38), 150 (57), 147 (69), 101 (80), 97 (88), 85 (100). HRMS (EI ${ }^{+}$) calcd for $\mathrm{C}_{16} \mathrm{H}_{30}{ }^{81} \mathrm{BrO}_{2}, 335.1409$ and $\mathrm{C}_{16} \mathrm{H}_{30}{ }^{79} \mathrm{BrO}_{2}, 333.1429$; found 335.1410 and 333.1443 .

2-(12-Bromododecyloxy)-tetrahydro-2H-pyran (4b). Following the same procedure described for $\mathbf{4 a}$, protected alcohol $\mathbf{4 b}$ was obtained in $93 \%$ yield. ${ }^{1} \mathrm{H}$ NMR $\left(400 \mathrm{MHz}, \mathrm{CDCl}_{3}\right) \delta 4.57$ (broad s, $\left.1 \mathrm{H}\right), 3.9-3.8(\mathrm{~m}, 1 \mathrm{H}), 3.8-3.7(\mathrm{~m}, 1 \mathrm{H}), 3.5-3.4(\mathrm{~m}$, 1H), $3.40(\mathrm{t}, J=6.9 \mathrm{~Hz}, 2 \mathrm{H}), 3.4-3.3(\mathrm{~m}, 1 \mathrm{H}), 1.9-1.8(\mathrm{~m}, 3 \mathrm{H}), 1.7-1.6(\mathrm{~m}, 1 \mathrm{H}), 1.6-1.4$ (m, 6H), 1.4-1.2 (m, 16H) ppm. ${ }^{13} \mathrm{C}$ NMR $\left(100 \mathrm{MHz}, \mathrm{CDCl}_{3}\right) \delta$ 98.8, 67.6, 62.3, 34.0, $32.8,30.7,29.7,29.6,29.5,29.4,29.3,28.7,28.1,26.2,25.5,19.7 \mathrm{ppm} . \mathrm{IR}(\mathrm{NaCl}) v$ 2913 (s, C-H), 2849 (s, C-H), 1458 (m), 1349 (m), 1257 (w), 1200 (w), 1120 (s), 1075 (s), 1027 (s). MS (EI $) m / z(\%) 349$ ([M-1] $\left.{ }^{+}\left[{ }^{81} \mathrm{Br}\right], 1\right), 347\left([\mathrm{M}-1]^{+}\left[{ }^{79} \mathrm{Br}\right], 1\right), 150$ (21), 
148 (22), 111 (13), 101 (24), 97 (74), 85 (100). HRMS $\left(\mathrm{EI}^{+}\right)$calcd for $\mathrm{C}_{17} \mathrm{H}_{32}{ }^{81} \mathrm{BrO}_{2}$, 349.1565 and $\mathrm{C}_{17} \mathrm{H}_{32}{ }^{79} \mathrm{BrO}_{2}, 347.1586$; found 349.1573 and 347.1601 .

3-[12-(Tetrahydro-2H-pyran-2-yloxy)dodecyl]pyridine (5a). $n$-BuLi (16.8 mL, $26.8 \mathrm{mmol}, 1.6 \mathrm{M}$ in hexanes) was added dropwise to a stirred solution of diisopropylamine $(3.8 \mathrm{~mL}, 26.80 \mathrm{mmol})$ in $\mathrm{THF}(17 \mathrm{~mL})$ at $0{ }^{\circ} \mathrm{C}$. The slightly yellow solution was stirred at $0{ }^{\circ} \mathrm{C}$ for $30 \mathrm{~min}$. DMPU (3.2 mL, $\left.26.8 \mathrm{mmol}\right)$ was added and stirring at $0{ }^{\circ} \mathrm{C}$ was continued for another $20 \mathrm{~min}$. A solution of 3-methylpyridine (2.6 $\mathrm{mL}, 26.8 \mathrm{mmol})$ in THF $(8 \mathrm{~mL})$ was added dropwise to the reaction mixture. The resulting deep red solution was stirred at $0{ }^{\circ} \mathrm{C}$ for further $30 \mathrm{~min}$. Then it was cooled down to $-78{ }^{\circ} \mathrm{C}$ and a solution of $\mathbf{4 a}(3.0 \mathrm{~g}, 8.95 \mathrm{mmol})$ in THF $(8 \mathrm{~mL})$ was added dropwise. The reaction mixture was stirred at $-78^{\circ} \mathrm{C}$ for $1 \mathrm{~h}$ and then it was allowed to reach slowly room temperature. The excess organolithium was quenched by the addition of a saturated $\mathrm{NH}_{4} \mathrm{Cl}$ solution $(15 \mathrm{~mL})$ and $\mathrm{H}_{2} \mathrm{O}(10 \mathrm{~mL})$. The mixture was extracted with EtOAc $(3 \times 70 \mathrm{~mL})$, the combined organic layers were dried over $\mathrm{Na}_{2} \mathrm{SO}_{4}$ and the solvents removed under reduced pressure. The residue was purified by flash chromatography (60:40, hexane-EtOAc) to afford $2.53 \mathrm{~g}$ (76\% yield) of the title compound as a colourless oil. ${ }^{1} \mathrm{H}$ NMR $\left(400 \mathrm{MHz}, \mathrm{CDCl}_{3}\right) \delta 8.42-8.41(\mathrm{~m}, 2 \mathrm{H}), 7.48(\mathrm{~d}$, $J=7.7 \mathrm{~Hz}, 1 \mathrm{H}), 7.19(\mathrm{dd}, J=7.7,4.8 \mathrm{~Hz}, 1 \mathrm{H}), 4.57(\operatorname{broad} \mathrm{s}, 1 \mathrm{H}), 3.9-3.8(\mathrm{~m}, 1 \mathrm{H}), 3.7-$ $3.6(\mathrm{~m}, 1 \mathrm{H}), 3.5-3.4(\mathrm{~m}, 1 \mathrm{H}), 3.4-3.3(\mathrm{~m}, 1 \mathrm{H}), 2.7-2.6(\mathrm{~m}, 1 \mathrm{H}), 1.9-1.8(\mathrm{~m}, 1 \mathrm{H}), 1.7-1.6$ (m, 1H), 1.6-1.4 (m, 8H), 1.4-1.12 (m, 16H) ppm. ${ }^{13} \mathrm{C}$ NMR (100 MHz, $\left.\mathrm{CDCl}_{3}\right) \delta$ 149.9, $147.1,137.9,135.6,123.1,98.8,67.6,62.3,32.9,31.1,30.7,29.7,29.5,29.4(2 x), 29.3$, 29.1, 26.2, 25.4, 19.6 ppm. IR (NaCl) v 2924 (s, C-H), 2851 (s, C-H), 1582 (m), 1461 (m), 1371 (m), 1119 (s), 1075 (s), 1026 (s), 982 (m), 712 (m). MS (EI $)$ m/z (\%) 346 $\left([\mathrm{M}-1]^{+}, 2\right), 318$ (7), 262 (97), 246 (100), 232 (38), 218 (32), 204 (51), 190 (38), 176 
(25), 148 (30), 106 (96), 93 (95). HRMS (EI ${ }^{+}$calcd for $\mathrm{C}_{22} \mathrm{H}_{36} \mathrm{NO}_{2}, 346.2746$; found 346.2739 .

3-[13-(Tetrahydro-2H-pyran-2-yloxy)tridecyl]pyridine (5b). Following the same procedure described for $\mathbf{5 a}$, pyridine $\mathbf{5 b}$ was obtained in $70 \%$ yield. ${ }^{1} \mathrm{H}$ NMR (400 $\left.\mathrm{MHz}, \mathrm{CDCl}_{3}\right) \delta 8.45-8.41(\mathrm{~m}, 2 \mathrm{H}), 7.48(\mathrm{~d}, J=7.7 \mathrm{~Hz}, 1 \mathrm{H}), 7.19(\mathrm{dd}, J=7.7,4.8 \mathrm{~Hz}$, 1H), 4.57 (broad s, 1H), 3.9-3.8 (m, 1H), 3.8-3.7 (m, 1H), 3.5-3.4 (m, 1H), 3.4-3.3 (1H), 2.7-2.6 (m, 1H), 1.9-1.8 (m, 1H), 1.7-1.6 (m, 1H), 1.6-1.5 (m, 8H), 1.42-1.2 (m, 18H) ppm ${ }^{13} \mathrm{C}$ NMR $\left(100 \mathrm{MHz}, \mathrm{CDCl}_{3}\right) \delta 149.8,147.0,137.7,135.5,123.0,98.6,67.5,62.1$, $32.8,30.9,30.6,29.6,29.4,29.3(2 \mathrm{x}), 29.2,29.0,26.1,25.3,19.5 \mathrm{ppm}$. IR $(\mathrm{NaCl}) v$ 2912 (s, C-H), 2849 (s, C-H), 1574 (w), 1459 (m), 1422 (m), 1349 (m), 1120 (s), 1074 (s), 1026 (s), 987 (m), 713 (m). MS (FAB $\left.{ }^{+}\right) \mathrm{m} / z(\%) 362\left([\mathrm{M}+1]^{+}, 45\right), 278$ (100), 260 (27). HRMS (EI $)$ calcd for $\mathrm{C}_{23} \mathrm{H}_{38} \mathrm{NO}_{2}, 360.2903$; found 360.2911 .

\section{1-(Oct-7-enyl)-3-[12-(tetrahydro-2H-pyran-2-yloxy)dodecyl]pyridinium Bromide}

(6a). 8-Bromo-1-octene (2 mL, $11.93 \mathrm{mmol}, 1$ equiv) was added to a solution of $\mathbf{5 a}$ $(4.15 \mathrm{~g}, 11.93 \mathrm{mmol})$ in acetonitrile $(20 \mathrm{~mL})$. The resulting solution was heated at reflux and the disappearance of the starting material was monitored by TLC. After completion of the reaction (usually $24 \mathrm{~h}$ ), the solvent was removed under reduced pressure. The residue was purified by flash chromatography $\left(90: 10, \mathrm{CH}_{2} \mathrm{Cl}_{2}-\mathrm{MeOH}\right)$ to afford $6.16 \mathrm{~g}$ (96\% yield) of the title compound as a viscous oil. ${ }^{1} \mathrm{H}$ NMR $\left(400 \mathrm{MHz}, \mathrm{CDCl}_{3}\right) \delta 9.41$ $(\mathrm{d}, J=5.9 \mathrm{~Hz}, 1 \mathrm{H}), 9.29(\mathrm{~s}, 1 \mathrm{H}), 8.17(\mathrm{~d}, J=8.0 \mathrm{~Hz}, 1 \mathrm{H}), 8.03(\mathrm{dd}, J=8.0,5.9 \mathrm{~Hz}$, 1H), 5.7-5.6 (m, 1H), $4.94(\mathrm{t}, J=7.4 \mathrm{~Hz}, 2 \mathrm{H}), 4.9-4.8(\mathrm{~m}, 2 \mathrm{H}), 4.5-4.4(\mathrm{~m}, 1 \mathrm{H}), 3.80-$ 3.7- $(\mathrm{m}, 1 \mathrm{H}), 3.7-3.6(\mathrm{~m}, 1 \mathrm{H}), 3.5-3.4(\mathrm{~m}, 1 \mathrm{H}), 3.3-3.2(\mathrm{~m}, 1 \mathrm{H}), 2.83(\mathrm{t}, J=6.9 \mathrm{~Hz}$, 2H), 2.0-1.9 (m, 4H), 1.8-1.7 (m, 1H), 1.7-1.6 (m, 3H), 1.5-1.4 (m, 6H), 1.4-1.1 (m, 22H) ppm. ${ }^{13} \mathrm{C} \mathrm{NMR}\left(100 \mathrm{MHz}, \mathrm{CDCl}_{3}\right) \delta 144.3,143.8,143.6,142.6,138.3,127.9$, 114.2, 98.6, 67.4, 62.1, 61.4, 33.3, 32.2, 31.6, 30.5, 30.1, 29.4, 29.2, 29.1, 28.9, 28.7, 
28.2, 28.1, 25.9, 25.5, 25.1, 19.4 ppm. IR (NaCl) v 2924 (s, C-H), 2852 (s, C-H), 1635

(m), 1503 (m), 1461 (m), 1351 (w), 1120 (s), 1075 (s), 1026 (s), 906 (m), 690 (m). MS

$\left(\mathrm{EI}^{+}\right) m / z(\%) 458\left(\left[\mathrm{M}-\mathrm{Br}^{-}\right]^{+}, 4\right), 374$ (1), 262 (56), 246 (95), $106(100)$.

1-(Oct-7-enyl)-3-[13-(tetrahydro-2H-pyran-2-yloxy)tridecyl]pyridinium Bromide

(6b). Following the same procedure described for $\mathbf{6 a}$, pyridinium salt $\mathbf{6 b}$ was obtained in $98 \%$ yield. ${ }^{1} \mathrm{H}$ NMR $\left(400 \mathrm{MHz}, \mathrm{CDCl}_{3}\right) \delta 9.37(\mathrm{~d}, J=5.2 \mathrm{~Hz}, 1 \mathrm{H}), 9.14(\mathrm{~s}, 1 \mathrm{H}), 8.21$ $(\mathrm{d}, J=7.7 \mathrm{~Hz}, 1 \mathrm{H}) 8.01(\mathrm{dd}, J=7.7,6.3 \mathrm{~Hz}, 1 \mathrm{H}), 5.7-5.6(\mathrm{~m}, 1 \mathrm{H}), 5.02(\mathrm{t}, J=7.4 \mathrm{~Hz}$, 2H), 5.0-4.9 (m, 2H), 3.9-3.8 (m, 1H), 3.7-3.6 (m, 1H), 3.5-3.4 (m, 1H), 3.4-3.3 (m, 1H), $2.89(\mathrm{~d}, J=7.8 \mathrm{~Hz}, 2 \mathrm{H}), 2.1-1.9(\mathrm{~m}, 4 \mathrm{H}), 1.9-1.8(\mathrm{~m}, 1 \mathrm{H}), 1.7-1.6(\mathrm{~m}, 3 \mathrm{H}), 1.6-1.5$ $(\mathrm{m}, 6 \mathrm{H}), 1.4-1.2(\mathrm{~m}, 24 \mathrm{H}) \mathrm{ppm} .{ }^{13} \mathrm{C} \mathrm{NMR}\left(100 \mathrm{MHz}, \mathrm{CDCl}_{3}\right) \delta 144.5,144.1,143.8$, $142.7,138.5,127.8,114.5,98.8,67.6,62.3,61.8,33.4,32.7,31.9,30.7,30.4,29.7$, 29.5, 29.4, 29.2, 29.0, $28.4(2 \mathrm{x}), 26.1,25.8,25.4,19.7$ ppm. IR (NaCl) v2925 (s, C-H), 2853 (s, C-H), 1637 (m), 1505 (m), 1465 (m), 1352 (w), 1136 (m), 1120 (s), 1032 (s), $906(\mathrm{~m}), 691(\mathrm{~m}) . \mathrm{MS}\left(\mathrm{FAB}^{+}\right) \mathrm{m} / z(\%) 472\left(\left[\mathrm{M}-\mathrm{Br}^{-}\right]^{+}, 100\right)$. HRMS $\left(\mathrm{FAB}^{+}\right)$calcd for $\mathrm{C}_{31} \mathrm{H}_{54} \mathrm{NO}_{2}{ }^{+}, 472.4155$; found 472.4144 .

3-(12-Hydroxydodecyl)-1-(oct-7-enyl)pyridinium Bromide (7a). A 3M aqueous solution of $\mathrm{HCl}(4.4 \mathrm{~mL}, 1.1$ equiv) was added to a solution of $\mathbf{6 a}(6.0 \mathrm{~g}, 11.14 \mathrm{mmol})$ in $\mathrm{MeOH}(44 \mathrm{~mL})$. The reaction mixture was stirred at $25{ }^{\circ} \mathrm{C}$ for $16 \mathrm{~h}$. Most of the solvents were removed under reduced pressure to give a viscous oil. The residue was disolved in dichloromethane $(80 \mathrm{~mL})$ and washed with a saturated $\mathrm{NaHCO}_{3}$ aqueous solution $(80 \mathrm{~mL})$. The organic layer was dried with $\mathrm{Na}_{2} \mathrm{SO}_{4}$ and the solvents were removed under reduced pressure. The residue was purified by flash chromatography (90:10, $\left.\mathrm{CH}_{2} \mathrm{Cl}_{2}-\mathrm{MeOH}\right)$ to afford $4.45 \mathrm{~g}$ (88\% yield) of the title compound as a white solid. ${ }^{1} \mathrm{H}$ NMR $\left(400 \mathrm{MHz}, \mathrm{CDCl}_{3}\right) \delta 9.33(\mathrm{~s}, 1 \mathrm{H}), 9.28(\mathrm{~d}, J=5.8 \mathrm{~Hz}, 1 \mathrm{H}), 8.20(\mathrm{~d}, J=$ $8.0 \mathrm{~Hz}, 1 \mathrm{H}), 8.04(\mathrm{dd}, J=8.0,5.8 \mathrm{~Hz}, 1 \mathrm{H}), 5.67(\mathrm{tdd}, J=13.2,10.0,6.7 \mathrm{~Hz}, 1 \mathrm{H}), 4.9-$ 
$4.8(\mathrm{~m}, 4 \mathrm{H}), 3.52(\mathrm{t}, J=6.7 \mathrm{~Hz}, 2 \mathrm{H}), 2.83(\mathrm{t}, J=7.8 \mathrm{~Hz}, 2 \mathrm{H}), 2.4-2.3(\mathrm{~m}, 1 \mathrm{H}), 2.0-1.9$ $(\mathrm{m}, 4 \mathrm{H}), 1.7-1.56(\mathrm{~m}, 2 \mathrm{H}), 1.5-1.4(\mathrm{~m}, 2 \mathrm{H}), 1.4-1.1(\mathrm{~m}, 23 \mathrm{H}) \mathrm{ppm} .{ }^{13} \mathrm{C}$ NMR $(100$ $\left.\mathrm{MHz}, \mathrm{CDCl}_{3}\right) \delta 144.5,144.0,143.9,142.4,138.4,127.9,114.3,62.4,61.4,33.3,32.5$, $32.4,31.7,30.2,29.2(2 x), 28.9,28.7,28.3,28.2,25.6,25.5$ ppm. IR $(\mathrm{NaCl}) \vee 3500-$ 3100 (br, O-H), 2918 (s, C-H), 2847 (s, C-H), 1634 (m), 1513 (m), 1465 (m), 1058 (s), 922 (m). MS (EI+) m/z (\%) 374 ([M-Br- $\left.]^{+}, 12\right), 262$ (10), 246 (15), 232 (11), 204 (12), 190 (12), 120 (11), 106 (100). HRMS (EI ${ }^{+}$) calcd for $\mathrm{C}_{25} \mathrm{H}_{44} \mathrm{NO}_{2}{ }^{+}, 374.3423$; found 374.3430.

3-(13-Hydroxytridecyl)-1-(oct-7-enyl)pyridinium Bromide (7b). Following the same procedure described for $\mathbf{7 a}$, alcohol $\mathbf{7 b}$ was obtained in $82 \%$ yield. ${ }^{1} \mathrm{H}$ NMR (400 $\left.\mathrm{MHz}, \mathrm{CDCl}_{3}\right) \delta 9.35(\mathrm{~d}, J=6.0 \mathrm{~Hz}, 1 \mathrm{H}), 9.29(\mathrm{~s}, 1 \mathrm{H}), 8.21(\mathrm{~d}, J=8.0 \mathrm{~Hz}, 1 \mathrm{H}), 8.05$ $(\mathrm{dd}, J=8.0,6.0 \mathrm{~Hz}, 1 \mathrm{H}), 5.72(\mathrm{tdd}, J=16.9,10.2,6.7 \mathrm{~Hz}, 1 \mathrm{H}), 4.95(\mathrm{t}, J=7.4, \mathrm{~Hz}$, 2H), 4.93-4.86 (m, 2H), 3.59 (t, $J=6.6 \mathrm{~Hz}, 1 \mathrm{H}), 2.9-2.8(\mathrm{~m}, 2 \mathrm{H}), 2.1-1.9(\mathrm{~m}, 6 \mathrm{H}), 1.7-$ $1.6(\mathrm{~m}, 2 \mathrm{H}), 1.6-1.5(\mathrm{~m}, 2 \mathrm{H}), 1.4-1.1(\mathrm{~m}, 25 \mathrm{H}) \mathrm{ppm} .{ }^{13} \mathrm{C} \mathrm{NMR}\left(100 \mathrm{MHz}, \mathrm{CDCl}_{3}\right) \delta$ $144.5,144.0,143.9,142.4,138.4,127.9,114.3,62.4,61.4,33.3,32.5,32.4,31.7,30.2$, $29.2(2 \mathrm{x}), 28.9,28.7,28.3,28.2,25.6,25.5$ ppm. IR (NaCl) $v$ 3500-3100 (br, O-H), 2922 (s, C-H), 2848 (s, C-H), 1636 (m), 1513 (m), 1466 (m), 1306 (w), 1172 (w), 1056

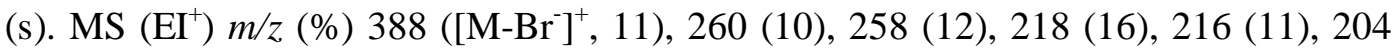
(15), 150 (40), 148 (41), 106 (100). HRMS (EI ${ }^{+}$) calcd for $\mathrm{C}_{26} \mathrm{H}_{46} \mathrm{NO}_{2}{ }^{+}, 388.3579$; found 374.3585 .

3-(12-Chlorododecyl)-1-(oct-7-enyl)pyridinium Chloride (8a). Thionyl chloride (0.95 mL, $13.02 \mathrm{mmol})$ was added to a solution of $7 \mathbf{a}(4.1 \mathrm{~g}, 10.02 \mathrm{mmol})$ in dichloromethane $(37 \mathrm{~mL})$ at $0{ }^{\circ} \mathrm{C}$. The mixture was maintained at $0{ }^{\circ} \mathrm{C}$ for $30 \mathrm{~min}$ and then it was stirred at $25^{\circ} \mathrm{C}$ for $6 \mathrm{~h}$. Dichloromethane $(60 \mathrm{~mL})$ was added and the resulting solution was washed with a $0.2 \mathrm{~N} \mathrm{NaOH}$ solution $(2 \times 50 \mathrm{~mL})$ and brine $(2 \mathrm{x}$ 
$50 \mathrm{~mL}$ ). The organic layer was dried over $\mathrm{Na}_{2} \mathrm{SO}_{4}$ and the solvents were removed under reduced pressure. The crude residue was purified by flash chromatography (90:10, $\left.\mathrm{CH}_{2} \mathrm{Cl}_{2}-\mathrm{MeOH}\right)$ to afford $3.9 \mathrm{~g}(91 \%)$ yield of the title compound as a viscous oil. ${ }^{1} \mathrm{H}$ NMR (400 MHz, $\left.\mathrm{CDCl}_{3}\right) \delta 9.44(\mathrm{~d}, J=5.9 \mathrm{~Hz}, 1 \mathrm{H}), 9.17(\mathrm{~s}, 1 \mathrm{H}), 8.19(\mathrm{~d}, J=7.9 \mathrm{~Hz}$, 1H), $8.01(\mathrm{dd}, J=7.9,5.9 \mathrm{~Hz}, 1 \mathrm{H}), 5.75(\mathrm{tdd}, J=16.9,10.2,6.7 \mathrm{~Hz}, 1 \mathrm{H}), 5.01(\mathrm{t}, J=$ $7.4 \mathrm{~Hz}, 2 \mathrm{H}), 5.0-4.9(\mathrm{~m}, 2 \mathrm{H}), 3.52(\mathrm{t}, J=6.8, \mathrm{~Hz}, 2 \mathrm{H}), 2.87(\mathrm{t}, J=6.9 \mathrm{~Hz}, 2 \mathrm{H}), 2.1-1.9$ $(\mathrm{m}, 4 \mathrm{H}), 1.9-1.7(\mathrm{~m}, 4 \mathrm{H}), 1.5-1.1(\mathrm{~m}, 22 \mathrm{H}) \mathrm{ppm} .{ }^{13} \mathrm{C} \mathrm{NMR}\left(100 \mathrm{MHz}, \mathrm{CDCl}_{3}\right) \delta$ 144.4, $144.0,143.9,142.7,138.4,127.9,114.4,61.6,45.1,33.3,32.6,32.4,31.9,30.3,29.3$ (2x), 29.2, 29.1, 28.9, 28.7, $28.3(2 \mathrm{x}), 26.7,25.7$ ppm. IR (NaCl) v 2924 (s, C-H), 2853 (s, C-H), 1637 (m), 1504 (m), 1462 (m), 1154 (m), 925 (m), 726 (s). MS (EI ${ }^{+}$m/z (\%) $394\left(\left[\mathrm{M}-\mathrm{Cl}^{-}\right]^{+}\left[{ }^{37} \mathrm{Cl}\right], 8\right) 392\left(\left[\mathrm{M}-\mathrm{Cl}^{-}\right]^{+}\left[{ }^{35} \mathrm{Cl}\right], 48\right), 356$ (7), 246 (94), 218 (15), 204 (18), 106 (100). HRMS (EI ${ }^{+}$) calcd for $\mathrm{C}_{25} \mathrm{H}_{43}{ }^{35} \mathrm{ClN}^{+}, 392.3084$ and $\mathrm{C}_{25} \mathrm{H}_{43}{ }^{37} \mathrm{ClN}^{+}, 394.3055$; found 392.3082 and 394.3073 .

3-(13-Chlorotridecyl)-1-(oct-7-enyl)pyridinium Chloride (8b). Following the same procedure, pyridinium chloride $\mathbf{8 b}$ was obtained in $95 \%$ yield. ${ }^{1} \mathrm{H}$ NMR $(400 \mathrm{MHz}$, $\left.\mathrm{CDCl}_{3}\right) \delta 9.37(\mathrm{~d}, J=5.9 \mathrm{~Hz}, 1 \mathrm{H}), 9.27(\mathrm{~s}, 1 \mathrm{H}), 8.21(\mathrm{~d}, J=7.8 \mathrm{~Hz}, 1 \mathrm{H}), 8.1-8.0(\mathrm{~m}$, 1H), 5.8-5.7 (m, 1H), 5.0-4.9 (m, 2H), 4.9-4.8 (m, 2H), 3.5-3.4 (m, 2H), 2.9-2.8 (m, 2H), 2.1-1.9 (m, 4H), 1.8-1.6 (m, 4H), 1.5-1.1 (m, 24H) ppm. ${ }^{13} \mathrm{C}$ NMR (100 MHz, $\left.\mathrm{CDCl}_{3}\right) \delta 144.2,143.8,143.7,142.2,138.1,127.6,114.0,61.1,44.8,33.0,32.1,31.5$ 30.0, $29.0(2 \mathrm{x}), 28.9,28.8,28.6,28.3,28.0(2 \mathrm{x}), 26.3,25.4 \mathrm{ppm} . \mathrm{IR}(\mathrm{NaCl}) \vee 2925(\mathrm{~s}$, C-H), 2853 (s, C-H), 1633 (m), 1504 (m), 1463 (m), 1154 (m), 726 (s). MS (EI ${ }^{+}$m/z

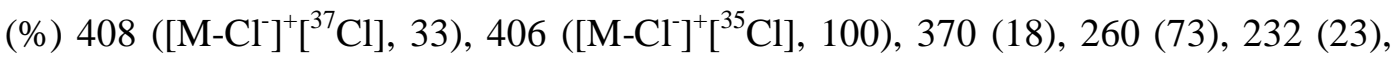
174 (21), 150 (52), 148 (50). HRMS (EI $)$ calcd for $\mathrm{C}_{26} \mathrm{H}_{45}{ }^{35} \mathrm{ClN}^{+}, 406.3241$ and $\mathrm{C}_{26} \mathrm{H}_{45}{ }^{37} \mathrm{ClN}^{+}$, 408.3211; found 406.3235 and 408.3209.

Preparation of pyridines 9a-c. See procedure for pyridines 5a,b 
3-(Pent-4-enyl)pyridine (9a). ${ }^{1} \mathrm{H}$ NMR (400 MHz, $\left.\mathrm{CDCl}_{3}\right) \delta 8.5-8.40$ (m, 2H), 7.48 $(\mathrm{d}, J=7.8 \mathrm{~Hz}, 1 \mathrm{H}), 7.19(\mathrm{dd}, J=7.8,4.9 \mathrm{~Hz}, 1 \mathrm{H}), 5.81(\mathrm{tdd}, J=16.9,10.2,6.7 \mathrm{~Hz}$, 1H), 5.0-4.9 (m, 2H), 2.7-2.6 (m, 2H), 2.1-2.0 (m, 2H), 1.8-1.7 (m, 2H) ppm. ${ }^{13} \mathrm{C}$ NMR $\left(100 \mathrm{MHz}, \mathrm{CDCl}_{3}\right) \delta 149.9,147.2,138.0,137.4,135.7,123.2,115.0,33.0,32.2,30.1$ ppm. IR (NaCl) v 2932 (s, C-H), 2858 (w, C-H), 1640 (m), 1575 (m), 1478 (m), 1422 (s), 912 (s). MS (EI $\left.I^{+}\right) m / z(\%) 147\left([\mathrm{M}]^{+}, 9\right), 146\left([\mathrm{M}-1]^{+}, 4\right), 118(18), 105$ (100), 93, (20), 92 (57), 78 (14). HRMS (EI ${ }^{+}$) calcd for $\mathrm{C}_{10} \mathrm{H}_{13} \mathrm{~N}, 147.1048$; found 147.1050.

3-(Hex-5-enyl)pyridine (9b). ${ }^{1} \mathrm{H}$ NMR $\left(400 \mathrm{MHz}, \mathrm{CDCl}_{3}\right) \delta 8.5-8.4(\mathrm{~m}, 2 \mathrm{H}), 7.46(\mathrm{~d}$, $J=7.8 \mathrm{~Hz}, 1 \mathrm{H}), 7.17(\mathrm{dd}, J=7.8,4.9 \mathrm{~Hz}, 1 \mathrm{H}), 5.77(\mathrm{tdd}, J=16.9,10.1,6.7 \mathrm{~Hz}, 1 \mathrm{H})$, $4.98(\mathrm{dd}, J=17.1,1.4 \mathrm{~Hz}, 1 \mathrm{H}), 4.93(\mathrm{dd}, J=10.2,1.2 \mathrm{~Hz}, 1 \mathrm{H}), 2.59(\mathrm{t}, J=7.5 \mathrm{~Hz}$, 2H), 2.1-2.0 (m, 2H), 1.7-1.6 (m, 2H), 1.5-1.4 (m, 2H) ppm. ${ }^{13} \mathrm{C}$ NMR (100 MHz, $\left.\mathrm{CDCl}_{3}\right) \delta 149.8,147.1,138.4,137.6,135.6,123.1,114.5,33.4,32.7,30.4,28.2$ ppm. IR (NaCl) $v 2907(\mathrm{w}, \mathrm{C}-\mathrm{H}), 2858(\mathrm{w}, \mathrm{C}-\mathrm{H}), 1639(\mathrm{~m}), 1575(\mathrm{~m}), 1477$ (m), $1421(\mathrm{~s}), 1189$ (w), 1025 (m), 992 (m), 909 (s), 795 (m), 712 (s). MS (EI+) m/z (\%) $161\left([\mathrm{M}]^{++}, 3\right), 160$ (8), 137 (11), 133 (65), 118 (19), 105 (29), 92 (47), 81 (11), 69 (100). HRMS (EI $\left.{ }^{+}\right)$ calcd for $\mathrm{C}_{11} \mathrm{H}_{15} \mathrm{~N}$ 161.1204; found 161.1210.

3-(Oct-7-enyl)pyridine (9c). ${ }^{1} \mathrm{H}$ NMR $\left(400 \mathrm{MHz}, \mathrm{CDCl}_{3}\right) \delta$ 8.5-8.4 (m, 2H), 7.47 (d, $J=7.6 \mathrm{~Hz}, 1 \mathrm{H}), 7.19(\mathrm{dd}, J=7.6,4.9 \mathrm{~Hz}, 1 \mathrm{H}), 5.79(\mathrm{tdd}, J=17.0,10.2,6.7 \mathrm{~Hz}, 1 \mathrm{H})$, $4.98(\mathrm{dd}, J=17.1,1.3 \mathrm{~Hz}, 1 \mathrm{H}), 4.92(\mathrm{ddd}, J=10.2,2.0,1.0 \mathrm{~Hz}, 1 \mathrm{H}), 2.59(\mathrm{t}, J=7.1$ $\mathrm{Hz}, 2 \mathrm{H}), 2.1-2.0(\mathrm{~m}, 2 \mathrm{H}), 1.7-1.6(\mathrm{~m}, 1 \mathrm{H}), 1.4-1.3(\mathrm{~m}, 6 \mathrm{H}) \mathrm{ppm} .{ }^{13} \mathrm{C} \mathrm{NMR}(100 \mathrm{MHz}$, $\left.\mathrm{CDCl}_{3}\right) \delta 149.9,147.1,139.0,137.9,135.7,123.2,114.2,33.7,32.9,31.0,28.9,28.8$, 28.7 ppm. IR (NaCl) v 2925 (s, C-H), 2854 (w, C-H), 1639 (m), 1574 (m), 1477 (m), 1421 (s), 1188 (w), 1026 (m), 994 (m), 907 (s), 793 (m), 712 (s). MS (EI $\left.{ }^{+}\right)$m/z (\%) 189 $\left([\mathrm{M}]^{++}, 21\right), 188$ (25), 146 (100), 133 (27), 106 (27), 105 (19), 93 (29), 92 (63). 
1-(Oct-7-enyl)-3-\{12-[3-(oct-7-enyl)pyridinium-1-yl]dodecyl\}pyridinium Chloride Iodide (10c). ${ }^{1} \mathrm{H}$ NMR (400 MHz, $\left.\mathrm{CD}_{3} \mathrm{OD}\right) \delta 9.13$ (br s, 2H), 9.0-8.9 (m, 2H), 8.53 (d, $J=8.0 \mathrm{~Hz}, 2 \mathrm{H}), 8.07(\mathrm{dd}, J=8.0,6.3 \mathrm{~Hz}, 2 \mathrm{H}), 5.8-5.7(\mathrm{~m}, 2 \mathrm{H}), 4.98(\mathrm{~d}, J=17.1 \mathrm{~Hz}$, 2H), $4.91(\mathrm{~d}, J=10.1 \mathrm{~Hz}, 2 \mathrm{H}), 4.73(\mathrm{t}, J=7.6 \mathrm{~Hz}, 4 \mathrm{H}), 2.93(\mathrm{t}, J=7.7 \mathrm{~Hz}, 4 \mathrm{H}), 2.1-$ $2.0(\mathrm{~m}, 8 \mathrm{H}), 1.8-1.7(\mathrm{~m}, 4 \mathrm{H}), 1.5-1.2(\mathrm{~m}, 28 \mathrm{H}) \mathrm{ppm} .{ }^{13} \mathrm{C} \mathrm{NMR}\left(100 \mathrm{MHz}, \mathrm{CD}_{3} \mathrm{OD}\right) \delta$ $146.7,145.6,145.5,145.3,143.2,139.9,139.8,129.0,115.0,114.9,62.7,34.7,34.6$, $33.4(2 x), 32.5,31.5,31.4,30.7,30.6(2 x), 30.5,30.1,29.9,29.8(2 x), 29.7,29.5,26.9$ ppm. IR (NaCl) v 3015 (s), 2924 (s, C-H), 2853 (s, C-H), 1633 (m), 1504 (s), 1457 (s), 1153 (m), 910 s), 732 (s), 688 (s). HRMS (ESI ${ }^{+}$) m/z calcd for $\mathrm{C}_{38} \mathrm{H}_{62} \mathrm{IN}_{2}{ }^{+} 673.3951$ (found 673.3945). $\mathrm{C}_{38} \mathrm{H}_{62} \mathrm{~N}_{2}{ }^{2+} 273.2451$ (found 273.2447).

\section{1-(Oct-7-enyl)-3-\{13-[3-(pent-4-enyl)pyridinium-1-yl]tridecyl $\}$ pyridinium}

Chloride Iodide (10d). ${ }^{1} \mathrm{H}$ NMR (400 MHz, $\left.\mathrm{CD}_{2} \mathrm{Cl}_{2}\right) \delta 9.36$ (br s, $\left.1 \mathrm{H}\right), 9.34$ (br s, $\left.1 \mathrm{H}\right)$, $9.19(\mathrm{~d}, J=6.0 \mathrm{~Hz}, 1 \mathrm{H}), 9.16(\mathrm{~d}, J=6.0 \mathrm{~Hz}, 1 \mathrm{H}), 8.32(\mathrm{~d}, J=8.0 \mathrm{~Hz}, 2 \mathrm{H}), 8.05(\mathrm{dd}, J$ $=8.0,6.0 \mathrm{~Hz}, 2 \mathrm{H}), 5.9-5.7(\mathrm{~m}, 2 \mathrm{H}), 5.1-4.9(\mathrm{~m}, 4 \mathrm{H}), 4.85(\mathrm{t}, J=7.4 \mathrm{~Hz}, 4 \mathrm{H}), 2.9-2.8$ $(\mathrm{m}, 4 \mathrm{H}), 2.1-2.0(\mathrm{~m}, 2 \mathrm{H}), 2.06-1.9(\mathrm{~m}, 6 \mathrm{H}), 1.8-1.7(\mathrm{~m}, 4 \mathrm{H}), 1.5-1.1(\mathrm{~m}, 24 \mathrm{H}) \mathrm{ppm} .{ }^{13} \mathrm{C}$ NMR (100 MHz, $\left.\mathrm{CD}_{2} \mathrm{Cl}_{2}\right) \delta 145.2,144.2,144.0,143.9,143.8,142.2,142.1,138.8$, $137.5,128.0,115.4,114.2,61.5,61.4,33.5,32.8,32.4,31.8,31.7,30.3,29.4,29.3$, 29.2, 29.1, 29.0, $28.8(2 \mathrm{x}), 28.4,28.3,25.8,25.7 \mathrm{ppm} . \mathrm{IR}(\mathrm{NaCl}) \vee 3016(\mathrm{~m}), 2925(\mathrm{~s}$, C-H), 2853 (w, C-H), 1631 (m), 1503 (s), 1460 (m), 1152 (m), 911 (m), 688 (s). MS $\left(\mathrm{ESI}^{+}\right)(\%) 645\left(\left[\mathrm{M}-\mathrm{Cl}^{-}\right]^{+}\right.$, 5). HRMS $\left(\mathrm{ESI}^{+}\right) \mathrm{m} / z$ calcd for $\mathrm{C}_{36} \mathrm{H}_{58} \mathrm{IN}_{2}{ }^{+} 645.3639$ (found 645.3626), $\mathrm{C}_{36} \mathrm{H}_{58} \mathrm{~N}_{2}{ }^{2+} 259.2294$ (found 259.2290).

\section{3-(Hex-5-enyl)-1-\{13-[1-(oct-7-enyl)pyridinium-3-yl]tridecyl\}pyridinium}

Chloride Iodide (10e). ${ }^{1} \mathrm{H}$ NMR (400 MHz, $\left.\mathrm{CD}_{3} \mathrm{OD}\right) \delta 9.05$ (br s, 2H), 8.90 (t, $J=5.3$ $\mathrm{Hz}, 2 \mathrm{H}), 8.49(\mathrm{~d}, J=8.0 \mathrm{~Hz}, 2 \mathrm{H}), 8.04(\mathrm{dd}, J=7.8,6.2 \mathrm{~Hz}, 2 \mathrm{H}), 5.8-5-7(\mathrm{~m}, 2 \mathrm{H}), 5.0-$ $4.9(\mathrm{~m}, 4 \mathrm{H}), 4.66(\mathrm{t}, J=7.6 \mathrm{~Hz}, 4 \mathrm{H}), 2.9-2.8(\mathrm{~m}, 4 \mathrm{H}), 2.2-2.1(\mathrm{~m}, 2 \mathrm{H}), 2.0-1.9(\mathrm{~m}, 6 \mathrm{H})$, 
1.8-1.7 (m, 4H), 1.5-1.4 (m, 2H), 1.4-1.2 (m, 24H) ppm. ${ }^{13} \mathrm{C}$ NMR (100 MHz, $\left.\mathrm{CD}_{3} \mathrm{OD}\right)$ $\delta 146.7,145.7,145.6,145.3,143.4,143.3,139.9,139.6,129.0,115.4,115.0,62.8(2 x)$, $34.7,34.5,33.5,33.3,32.6,32.5,31.6,31.0,30.7,30.6(2 x), 30.5,30.4,30.1(2 x), 29.8$, 29.6, 29.4, 27.2, 27.0 ppm. IR (NaCl) v 3016 (m), 2925 (s, C-H), 2853 (s, C-H), 1631 (m), 1505 (s), 1460 (m), 1152 (m), 911 (m), 688 (s). HRMS (ESI $\left.{ }^{+}\right) \mathrm{m} / \mathrm{z}$ calcd for $\mathrm{C}_{37} \mathrm{H}_{60} \mathrm{IN}_{2}{ }^{+} 659.3795$ (found 659.3787), $\mathrm{C}_{37} \mathrm{H}_{60} \mathrm{~N}_{2}{ }^{2+} 266.2372$ (found 266.2369).

\section{1-(Oct-7-enyl)-3-\{13-[3-(oct-7-enyl)pyridinium-1-yl]tridecyl\}pyridinium Chloride}

Iodide (10f). ${ }^{1} \mathrm{H}$ NMR (400 MHz, $\left.\mathrm{CD}_{3} \mathrm{OD}\right) \delta 9.02$ (br s, 2H), 8.9-8.8 (m, 2H), 8.48 (d, $J$ $=7.7 \mathrm{~Hz}, 2 \mathrm{H}), 8.03(\mathrm{dd}, J=7.7,6.4 \mathrm{~Hz}, 2 \mathrm{H}), 5.8-5.7(\mathrm{~m}, 2 \mathrm{H}), 4.98(\mathrm{~d}, J=17.1 \mathrm{~Hz}$, 2H), $4.91(\mathrm{~d}, J=10.2 \mathrm{~Hz}, 2 \mathrm{H}), 4.65(\mathrm{t}, J=7.5 \mathrm{~Hz}, 4 \mathrm{H}), 2.9-2.8(\mathrm{~m}, 4 \mathrm{H}), 2.1-2.0(\mathrm{~m}$, $8 \mathrm{H}), 1.8-1.7(\mathrm{~m}, 4 \mathrm{H}), 1.5-1.2(\mathrm{~m}, 30 \mathrm{H}) \mathrm{ppm} .{ }^{13} \mathrm{C} \mathrm{NMR}\left(100 \mathrm{MHz}, \mathrm{CD}_{3} \mathrm{OD}\right) \delta$ 146.7, $145.8,145.7,145.3,143.3,140.0,139.9,129.0,115.0,114.9,62.9,34.8,34.7,33.5$, $32.5,32.4,31.5(2 x), 30.6(2 x), 30.4(2 x), 30.1,30.0,29.9(2 x) 29.8,29.5,27.1,26.9$ ppm. IR (NaCl) v 3016 (m), 2925 (s, C-H), 2853 (s, C-H), 1631 (m), 1503 (s), 1460 (m), 1152 (m), 911 (m), 688 (s). HRMS $\left(\mathrm{ESI}^{+}\right) \mathrm{m} / \mathrm{z}$ calcd for $\mathrm{C}_{39} \mathrm{H}_{64} \mathrm{IN}_{2}{ }^{+} 687.4109$ (found 687.4097), $\mathrm{C}_{39} \mathrm{H}_{64} \mathrm{~N}_{2}{ }^{2+} 280.2529$ (found 280.2524).

Cyclostellettamine A (1a). ${ }^{1} \mathrm{H}$ NMR (400 MHz, $\left.\mathrm{CD}_{3} \mathrm{OD}\right) \delta 8.95$ (br s, $2 \mathrm{H}, \mathrm{H} 2 / 2$ '), $8.85\left(\mathrm{~d}, J=5.5 \mathrm{~Hz}, 2 \mathrm{H}, \mathrm{H} 6 / 6\right.$ '), $8.46\left(\mathrm{~d}, J=7.9 \mathrm{~Hz}, 2 \mathrm{H}, \mathrm{H} 4 / 4^{\prime}\right), 8.02(\mathrm{dd}, J=7.9,5.5$ Hz, 2H, H5/5'), 4.61 (t, J = 7.5 Hz, 4H, H7/7'), 2.9-2.8 (m, 4H, H18/18'), 2.1-2.0 (m, 4H, H8/8'), 1.7-1.6 (m, 4H, H17/17'), 1.5-1.3 (m, 32H, H9-16/9'-16') ppm. ${ }^{13} \mathrm{C}$ NMR (100 MHz, $\left.\mathrm{CD}_{3} \mathrm{OD}\right) \delta 146.6\left(\mathrm{C} 4 / 4^{\prime}\right), 145.7$ (C3/3'), $145.3\left(\mathrm{C} 2 / 2^{\prime}\right), 143.4\left(\mathrm{C} 6 / 6^{\prime}\right), 129.0$ (C5/5'), 62.9 (C7/7'), 33.6 (C18/18'), 32.6 (C8/8'), 31.6 (C17/17'), 30.7, 30.6, 30.5 (C1015/10'-15'), 30.1 (C16/16'), 27.1 (C9/9') ppm. IR (NaCl) v 3020 (m), 2924 (s, C-H), 2853 (s, C-H), 1632 (m), 1505 (s), 1463 (m), 1155 (m), 732 (m), 692 (s). HRMS (ESI $\left.{ }^{+}\right)$ 
$m / z$ calcd for $\mathrm{C}_{34} \mathrm{H}_{56} \mathrm{IN}_{2}{ }^{+}, 619.3483$ (found 619.3475 ), $\mathrm{C}_{34} \mathrm{H}_{56} \mathrm{ClN}_{2}{ }^{+} 527.4126$ (found 527.4120), $\mathrm{C}_{34} \mathrm{H}_{56} \mathrm{~N}_{2}{ }^{2+} 246.2216$ (found 246.2221).

Cyclostellettamine B (1b). ${ }^{1} \mathrm{H}$ NMR (400 MHz, CD $\left.{ }_{3} \mathrm{OD}\right) \delta 8.99$ (br s, 2H, H2/2'), $8.88\left(\mathrm{~d}, J=5.5 \mathrm{~Hz}, 2 \mathrm{H}, \mathrm{H} 6 / 6^{\prime}\right), 8.48\left(\mathrm{~d}, J=7.8 \mathrm{~Hz}, 2 \mathrm{H}, \mathrm{H} 4 / 4^{\prime}\right), 8.04(\mathrm{dd}, J=7.8,5.5$ Hz 2H, H5/5'), 4.65 (t, $J=7.4$ Hz, 4H, H7/7'), 2.90 (t, $J=7.7$ Hz, 4H, H18/19'), 2.1-1.9 (m, 4H, H8/8'), 1.8-1.6 (m, 4H, H17/18'), 1.5-1.2 (m, 34H, H9-16/9'-17') ppm. ${ }^{13} \mathrm{C}$ NMR (100 MHz, CD 3 OD) $\delta 146.6$ (C4/4'), 145.7 (C3/3'), 145.3 (C2/2'), 143.4(C6/6'), $129.0\left(\mathrm{C} 5 / 5^{\prime}\right), 62.9\left(\mathrm{C} 7 / 7^{\prime}\right), 33.5\left(\mathrm{C} 18 / 19^{\prime}\right), 32.6\left(\mathrm{C} 8 / 8^{\prime}\right), 31.6\left(\mathrm{C} 17 / 18^{\prime}\right), 30.7,30.6,30.5$ (C10-15/10'-16'), 30.2 (C16/17'), 27.2 (C9/9') ppm. IR (NaCl) v3020 (m), 2924 (s, CH), 2853 (s, C-H), 1632 (m), 1505 (s), 1464 (m), 1155 (m), 691 (s). HRMS (ESI ${ }^{+}$) m/z calcd for $\mathrm{C}_{35} \mathrm{H}_{58} \mathrm{IN}_{2}{ }^{+} 633.3639$ (found 633.3632), $\mathrm{C}_{35} \mathrm{H}_{58} \mathrm{ClN}_{2}{ }^{+} 541.4283$ (found 541.4275), $\mathrm{C}_{35} \mathrm{H}_{58} \mathrm{~N}_{2}{ }^{2+} 253.2294$ (found 253.2295).

Cyclostellettamine E (1e). ${ }^{1} \mathrm{H}$ NMR (400 MHz, $\left.\mathrm{CD}_{3} \mathrm{OD}\right) \delta 8.96$ (br s, $2 \mathrm{H}, \mathrm{H} 2 / 2$ '), $8.86\left(\mathrm{~d}, J=5.5 \mathrm{~Hz}, 2 \mathrm{H}, \mathrm{H} 6 / 6^{\prime}\right), 8.46$ (d, $\left.J=7.8 \mathrm{~Hz}, 2 \mathrm{H}, \mathrm{H} 4 / 4^{\prime}\right), 8.0-7.9$ (m, 2H, H5/5'), $4.62\left(\mathrm{t}, J=7.5 \mathrm{~Hz}, 4 \mathrm{H}, \mathrm{H} 7 / 7^{\prime}\right), 2.89$ (t, $\left.J=7.8 \mathrm{~Hz}, 4 \mathrm{H}, \mathrm{H} 19 / 20^{\prime}\right), 2.0-1.9$ (m, 4H, H8/8'), 1.74 (t, $J=7.2 \mathrm{~Hz}, 4 \mathrm{H}, \mathrm{H} 18 / 19 '), 1.5-1.2$ (m, 36H, H9-17/9'-18') ppm. ${ }^{13} \mathrm{C}$ NMR (100 MHz, CD $\left.{ }_{3} \mathrm{OD}\right) \delta 146.7$ (C4/4'), 145.8 (C3/3'), 145.3 (C2/2'), 143.4 (C6/6'), 129.0 (C5/5'), 62.9 (C7/7'), $33.6\left(\mathrm{C} 19 / 20^{\prime}\right), 32.6\left(\mathrm{C} 8 / 8^{\prime}\right), 31.7$ (C18/19'), 30.7, 30.6, 30.5, 30.3 (C10-16/10'-17'), 30.2 (C17/18'), 27.2 (C9/9') ppm. IR (NaCl) v 3021 (m), 2925 (s, CH), 2853 (s, C-H), 1632 (m), 1505 (s), 1456 (s), 1155 (m), 1035 (m), 692 (s). HRMS $\left(\mathrm{ESI}^{+}\right.$) $\mathrm{m} / z$ calcd for $\mathrm{C}_{37} \mathrm{H}_{62} \mathrm{IN}_{2}{ }^{+} 661.3952$ (found 661.3949), $\mathrm{C}_{37} \mathrm{H}_{62} \mathrm{ClN}_{2}{ }^{+} 569.4596$ (found 569.4590), $\mathrm{C}_{37} \mathrm{H}_{62} \mathrm{~N}_{2}{ }^{2+} 267.2451$ (found 267.2452).

Cyclostellettamine G (1g). ${ }^{1} \mathrm{H}$ NMR (400 MHz, CD $\left.\mathrm{CD}_{3} \mathrm{OD}\right) \delta 8.96$ (br s, 2H, H2/2'), $8.85\left(\mathrm{~d}, J=6.2 \mathrm{~Hz}, 2 \mathrm{H}, \mathrm{H} 6 / 6^{\prime}\right), 8.47\left(\mathrm{~d}, J=8.1 \mathrm{~Hz}, 2 \mathrm{H}, \mathrm{H} 4 / 4^{\prime}\right), 8.02$ (dd, $J=8.1,6.2$ Hz, 2H, H5/5'), 4.62 (t, $\left.J=7.3 \mathrm{~Hz}, 4 \mathrm{H}, \mathrm{H} 7 / 7^{\prime}\right), 2.9-2.8$ (m, 4H, H17/18'), 2.1-1.9 (m, 
4H, H8/8'), 1.73 (t, J= 7.2 Hz, 4H, H16/17'), 1.3-1.2 (m, 30H, H9-14/9'-15') ppm. ${ }^{13} \mathrm{C}$ NMR (100 MHz, CD 3 OD) $\delta 146.7$ (C4/4'), 145.8 (C3/3'), 145.3 (C2/2'), 143.4 (C6/6'), 129.0 (C5/5'), 62.9 (C7/7'), 33.6 (C17/18'), 32.6 (C8/8'), $31.6(\mathrm{C} 16 / 17 '), 30.7,3.06$, 30.5, 30.4 (C10-14/C10'-15'), 30.2 (C15/16'), 27.2 (C9/9') ppm. IR (NaCl) v 3022 (m), 2922 (s, C-H), 2852 (s, C-H), 1632 (m), 1505 (s), 1464 (m), 1156 (m), 691 (s). HRMS $\left(\mathrm{ESI}^{+}\right) \mathrm{m} / \mathrm{z}$ calcd for $\mathrm{C}_{33} \mathrm{H}_{54} \mathrm{IN}_{2}{ }^{+}, 605.3326$ (found 605.3317), $\mathrm{C}_{33} \mathrm{H}_{54} \mathrm{ClN}_{2}{ }^{+} 513.3970$ (found 513.3964), $\mathrm{C}_{33} \mathrm{H}_{54} \mathrm{~N}_{2}{ }^{2+} 239.2138$ ( found 239.2140).

Cyclostellettamine analogue (12). ${ }^{1} \mathrm{H}$ NMR (400 MHz, $\left.\mathrm{CD}_{3} \mathrm{OD}\right) \delta 8.97$ (br s, 2H, H2/2'), $8.85\left(\mathrm{~d}, J=5.8 \mathrm{~Hz}, 2 \mathrm{H}, \mathrm{H} 6 / 6^{\prime}\right), 8.46(\mathrm{~d}, J=8.0 \mathrm{~Hz}, 2 \mathrm{H}, \mathrm{H} 4 / 4), 8.02(\mathrm{dd}, J=$ 8.0, 5.8 Hz, 2H, H5/5'), 4.62 (t, $\left.J=7.5 \mathrm{~Hz}, 4 \mathrm{H}, \mathrm{H} 7 / 7^{\prime}\right), 2.9-2.8$ (m, 4H, H19/17'), 2.01.9 (m, 4H, H8/8'), 1.74 (t, $J=7.0 \mathrm{~Hz}, 4 \mathrm{H}, \mathrm{H} 18 / 16$ '), 1.4-1.2 (m, 36H, H9-17/9'-15') ppm. ${ }^{13} \mathrm{C}$ NMR (100 MHz, CD $\left.{ }_{3} \mathrm{OD}\right) \delta 146.7$ (C4/4'), 145.8 (C3/3'), $145.3\left(\mathrm{C} 2 / 2^{\prime}\right), 143.4$ (C6/6'), 129.0 (C5/5'), 62.9 (C7/7'), 33.6 (C19/17'), 32.6 (C8/8'), 31.7 (C18/16'), 30.8, 30.7, 30. 6, 30.5, 30.4, 30.3, 30.2 (C10-16/10'-14'), 30.1 (C17/15'), 27.2 (C9/9') ppm. IR $(\mathrm{NaCl})$ v $3021(\mathrm{~m}), 2923$ (s, C-H), 2852 (s, C-H), 1632 (m), 1505 (s), 1464 (m), 1155 (m), 692 (s). HRMS $\left(\mathrm{ESI}^{+}\right) \mathrm{m} / z$ calcd for $\mathrm{C}_{34} \mathrm{H}_{56} \mathrm{IN}_{2}{ }^{+}, 619.3483$ (found 619.3474), $\mathrm{C}_{34} \mathrm{H}_{56} \mathrm{ClN}_{2}{ }^{+} 527.4126$ (found 527.4121), $\mathrm{C}_{34} \mathrm{H}_{56} \mathrm{~N}_{2}{ }^{2+}$ 246. 2216 (found 246.2219). 


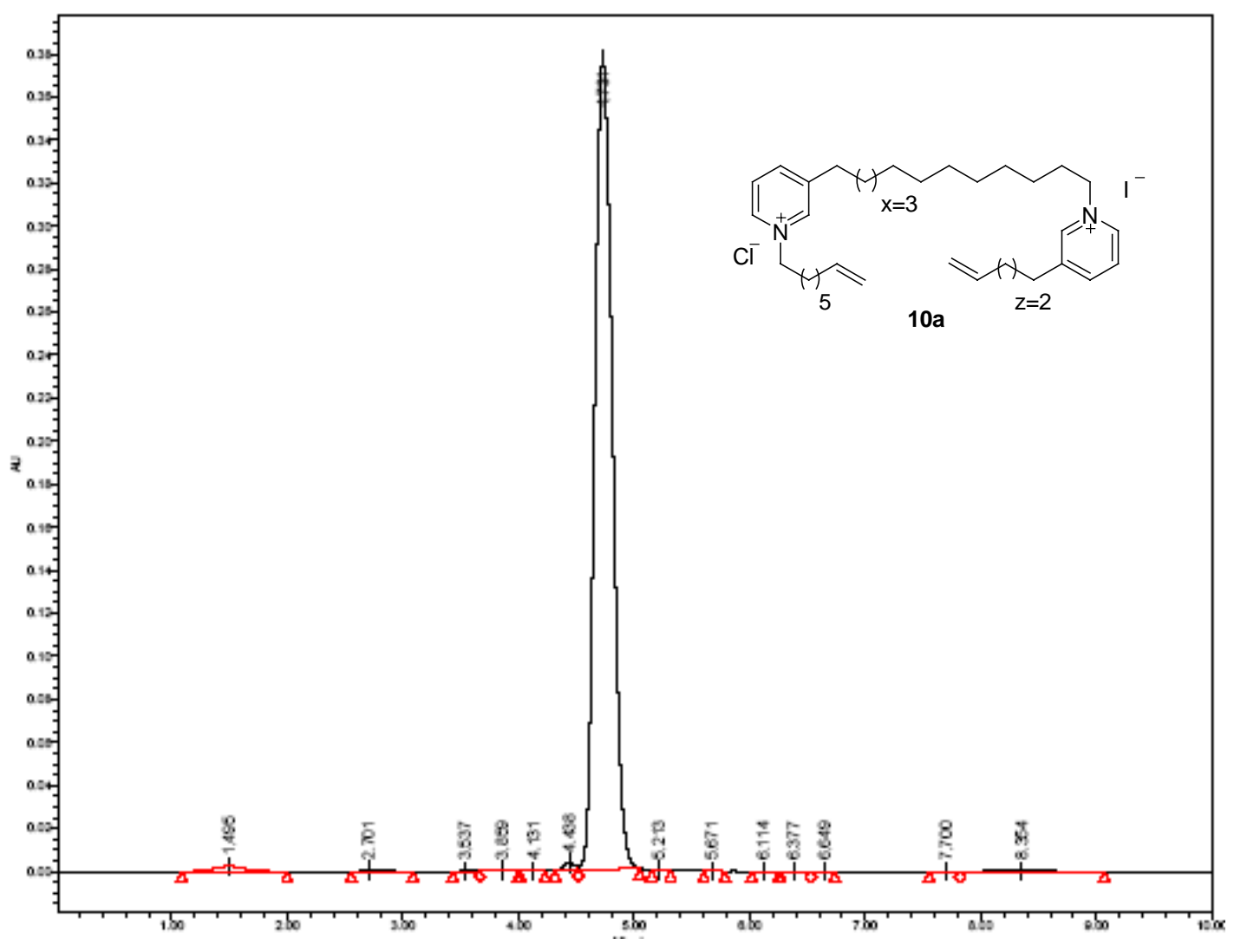

\begin{tabular}{|l|l|r|r|r|r|l|l|l|l|l|}
\hline & Name & $\begin{array}{c}\text { Retention Time } \\
(\mathrm{min})\end{array}$ & $\begin{array}{c}\text { Area } \\
\left(\mu \mathrm{V}^{*} \mathrm{sec}\right)\end{array}$ & \% Area & $\begin{array}{c}\text { Height } \\
(\mu \mathrm{V})\end{array}$ & Int Type & Amount & Units & Peak Type & Peak Codes \\
\hline 1 & & 1.495 & 43999 & 1.08 & 2352 & $\mathrm{BB}$ & & & Unknown & \\
\hline 2 & & 2.701 & 4523 & 0.11 & 527 & $\mathrm{BB}$ & & & Unknown & \\
\hline 3 & & 3.537 & 659 & 0.02 & 93 & $\mathrm{BV}$ & & & Unknown & \\
\hline 4 & & 3.859 & 5585 & 0.14 & 528 & VB & & & Unknown & \\
\hline 5 & & 4.131 & 868 & 0.02 & 157 & $\mathrm{BB}$ & & & Unknown & \\
\hline 6 & & 4.438 & 21834 & 0.54 & 3427 & $\mathrm{BV}$ & & & Unknown & \\
\hline 7 & & 4.731 & 3974704 & 97.78 & 377434 & VB & & & Unknown & \\
\hline 8 & & 5.213 & 624 & 0.02 & 130 & BB & & & Unknown & \\
\hline 8 & & 5.671 & 143 & 0.00 & 26 & BB & & & Unknown & \\
\hline 10 & & 6.114 & 210 & 0.01 & 28 & BB & & & Unknown & \\
\hline 11 & & 6.377 & 183 & 0.00 & 21 & BV & & & Unknown & \\
\hline 12 & & 6.649 & 247 & 0.01 & 39 & VB & & & Unknown & \\
\hline 13 & & 7.700 & 678 & 0.02 & 66 & BV & & & Unknown & \\
\hline 14 & & 8.354 & 10499 & 0.26 & 337 & VB & & & Unknown & \\
\hline
\end{tabular}

\section{Develosil C-30}

$\mathrm{H}_{2} \mathrm{O} / \mathrm{CH}_{3} \mathrm{CN}: 17 / 83$ (0.1\% TFA)

Flow: $3.2 \mathrm{~mL} / \mathrm{min}$

$\mathrm{v}=267 \mathrm{~nm}$ 

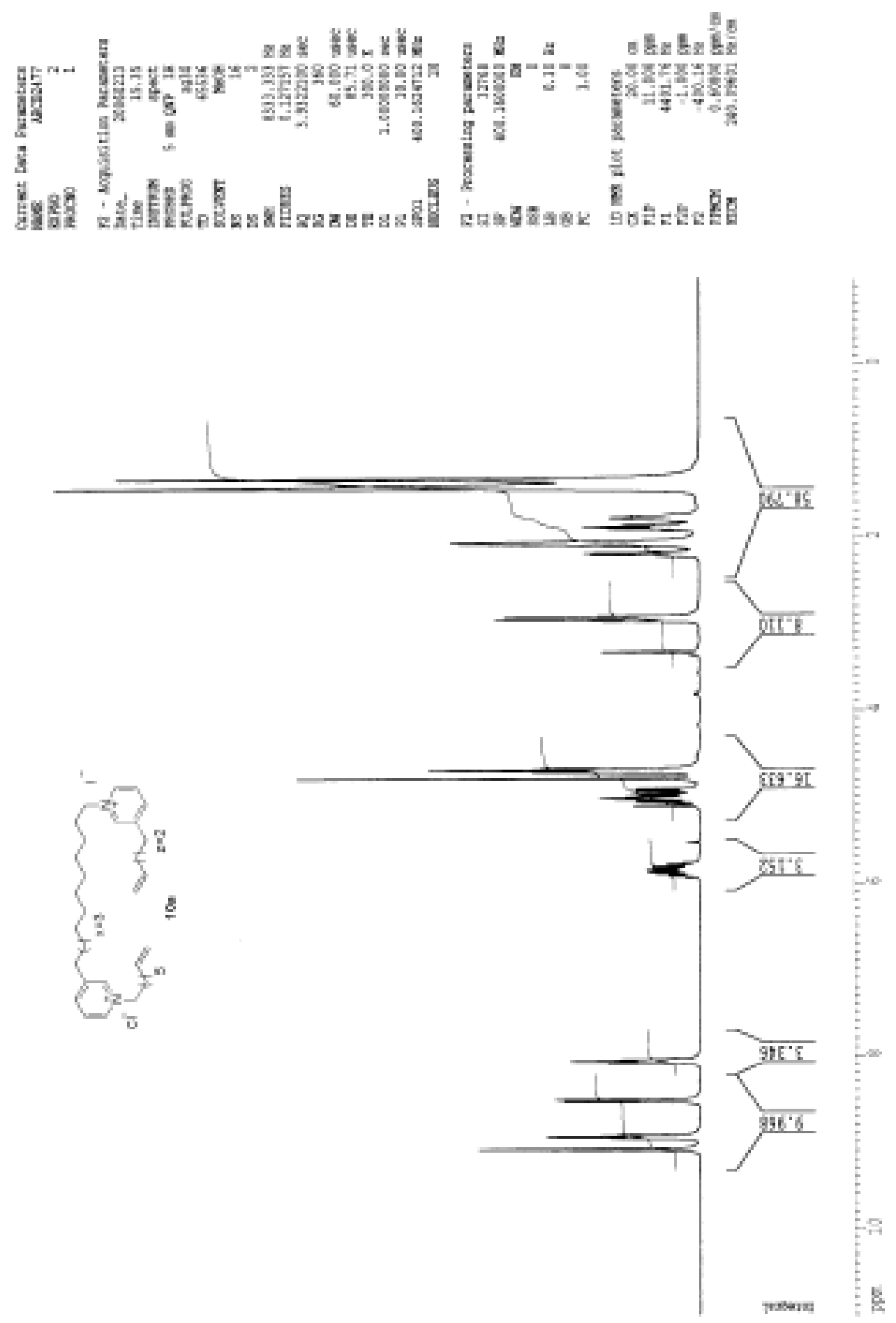

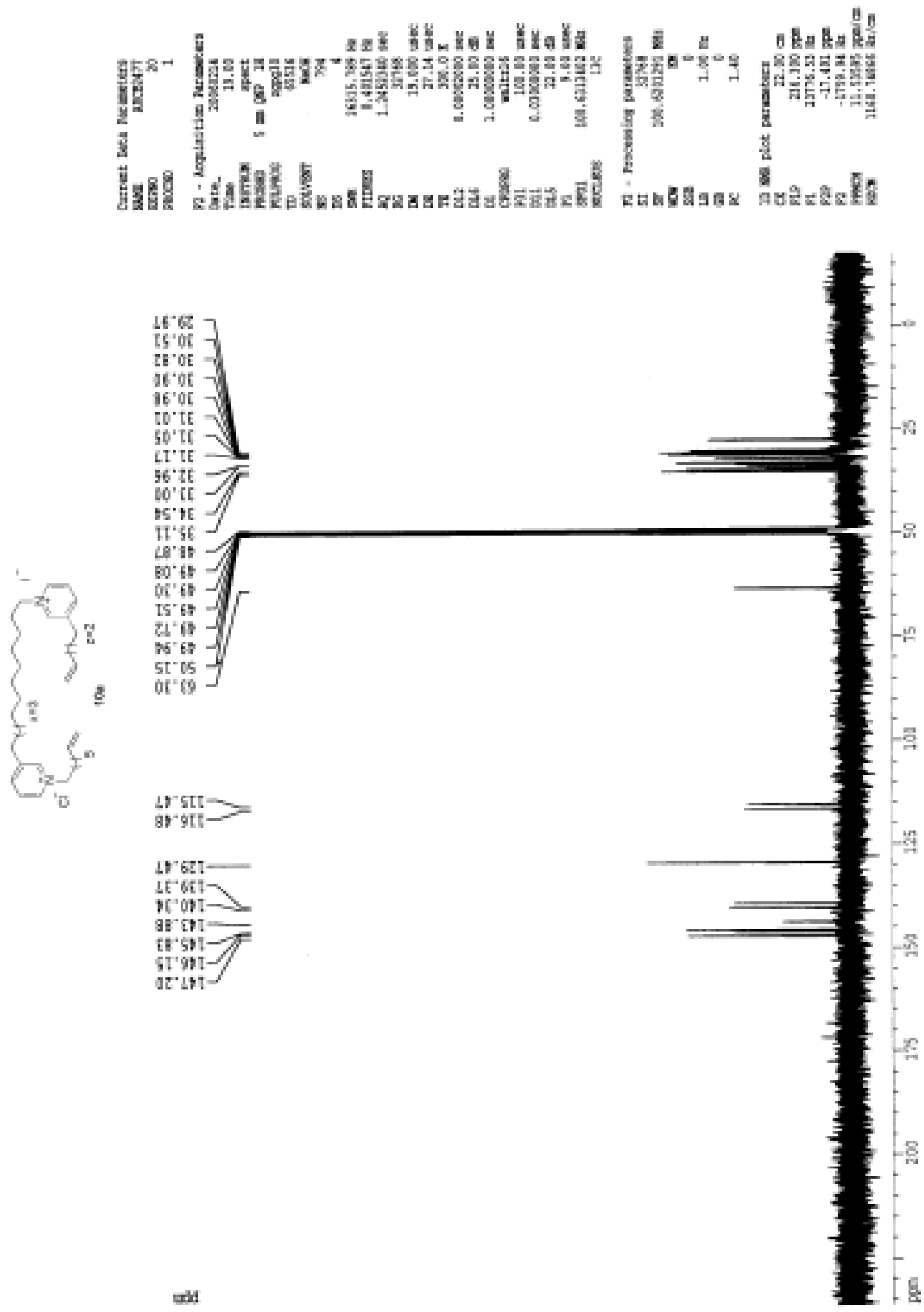


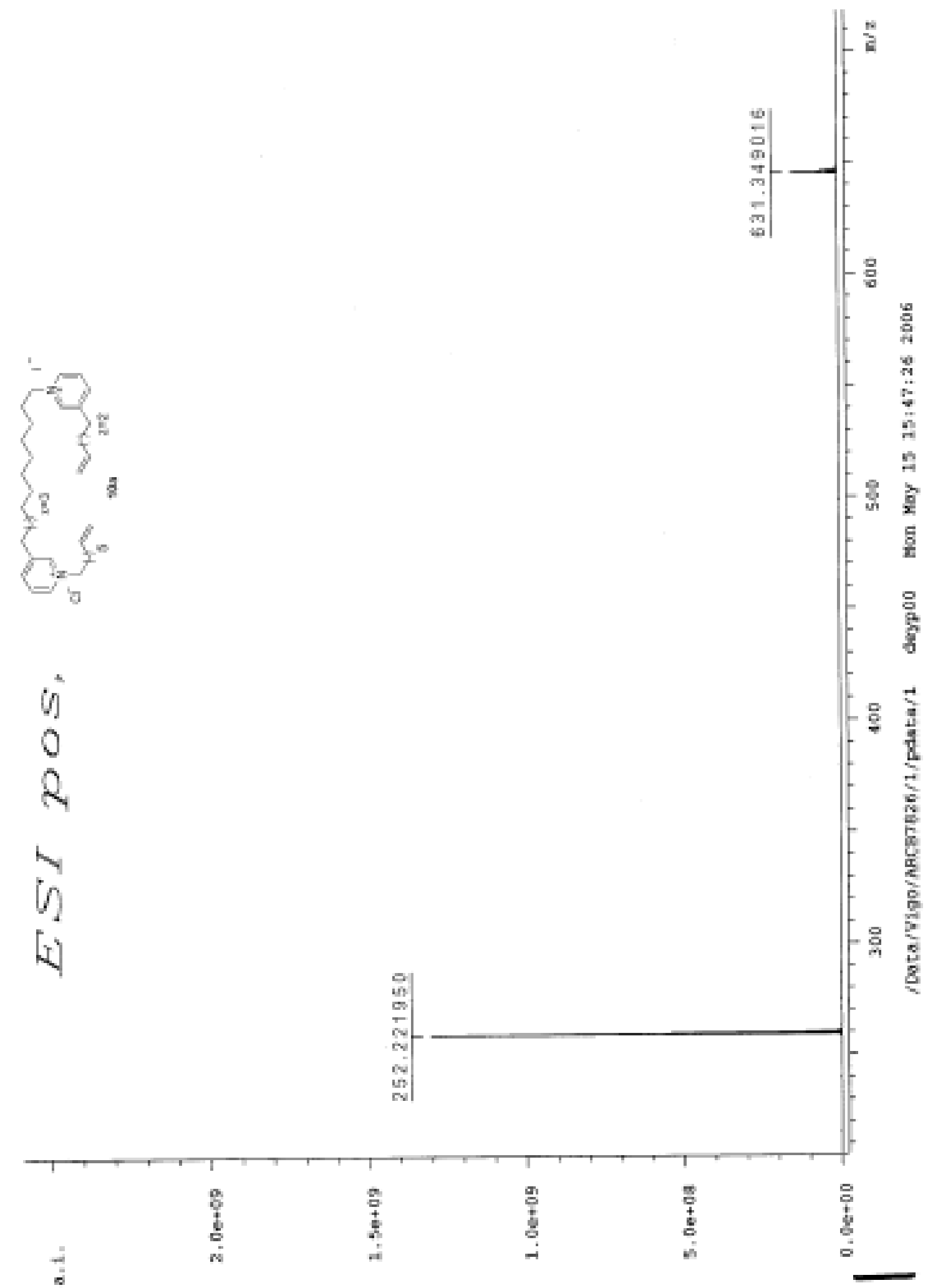




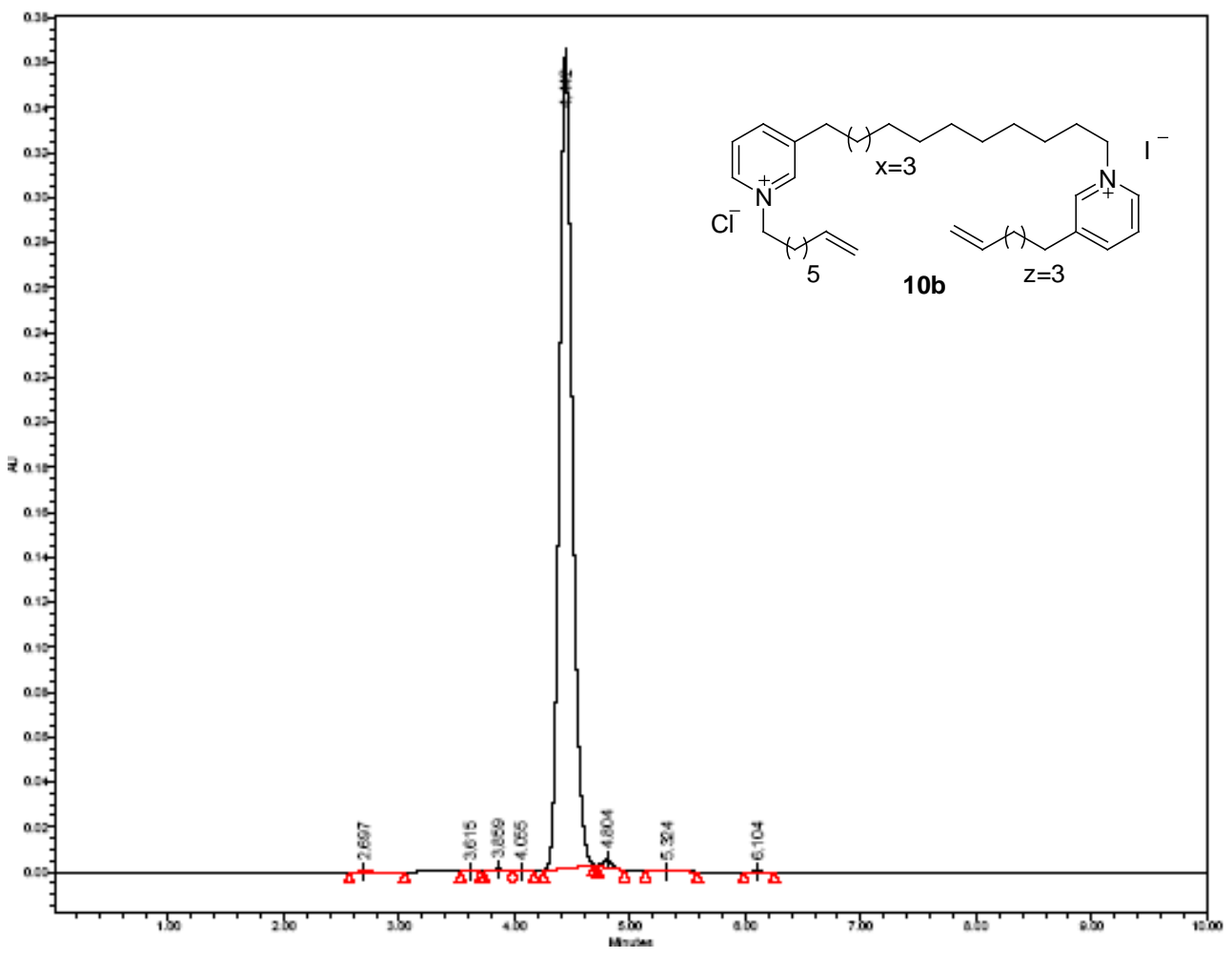

\begin{tabular}{|l|l|r|r|r|r|l|l|l|l|l|}
\hline & Name & $\begin{array}{c}\text { Retention Time } \\
(\mathrm{min})\end{array}$ & $\begin{array}{c}\text { Area } \\
\left(\mu \mathrm{V}^{\mathrm{x}} \mathrm{sec}\right)\end{array}$ & \% Area & $\begin{array}{c}\text { Height } \\
(\mu \mathrm{V})\end{array}$ & Int Type & Amount & Units & Peak Type & Peak Codes \\
\hline 1 & & 2.697 & 1123 & 0.04 & 108 & $\mathrm{BB}$ & & & Unknown & \\
\hline 2 & & 3.615 & 449 & 0.02 & 83 & $\mathrm{BB}$ & & & Unknown & \\
\hline 3 & & 3.859 & 7526 & 0.26 & 1067 & $\mathrm{BV}$ & & & Unknown & \\
\hline 4 & & 4.055 & 1347 & 0.05 & 190 & VB & & & Unknown & \\
\hline 5 & & 4.442 & 2863353 & 98.81 & 362979 & B B & & & Unknown & \\
\hline 8 & & 4.804 & 19824 & 0.68 & 3412 & BB & & & Unknown & \\
\hline 7 & & 5.324 & 3498 & 0.12 & 325 & BB & & & Unknown & \\
\hline 8 & & 6.104 & 752 & 0.03 & 97 & BB & & & Unknown & \\
\hline
\end{tabular}

Develosil C-30

$\mathrm{H}_{2} 0 / \mathrm{CH}_{3} \mathrm{CN}: 17 / 83$ (0.1\% TFA)

Flow: $3.2 \mathrm{~mL} / \mathrm{min}$

$\mathrm{v}=267 \mathrm{~nm}$ 

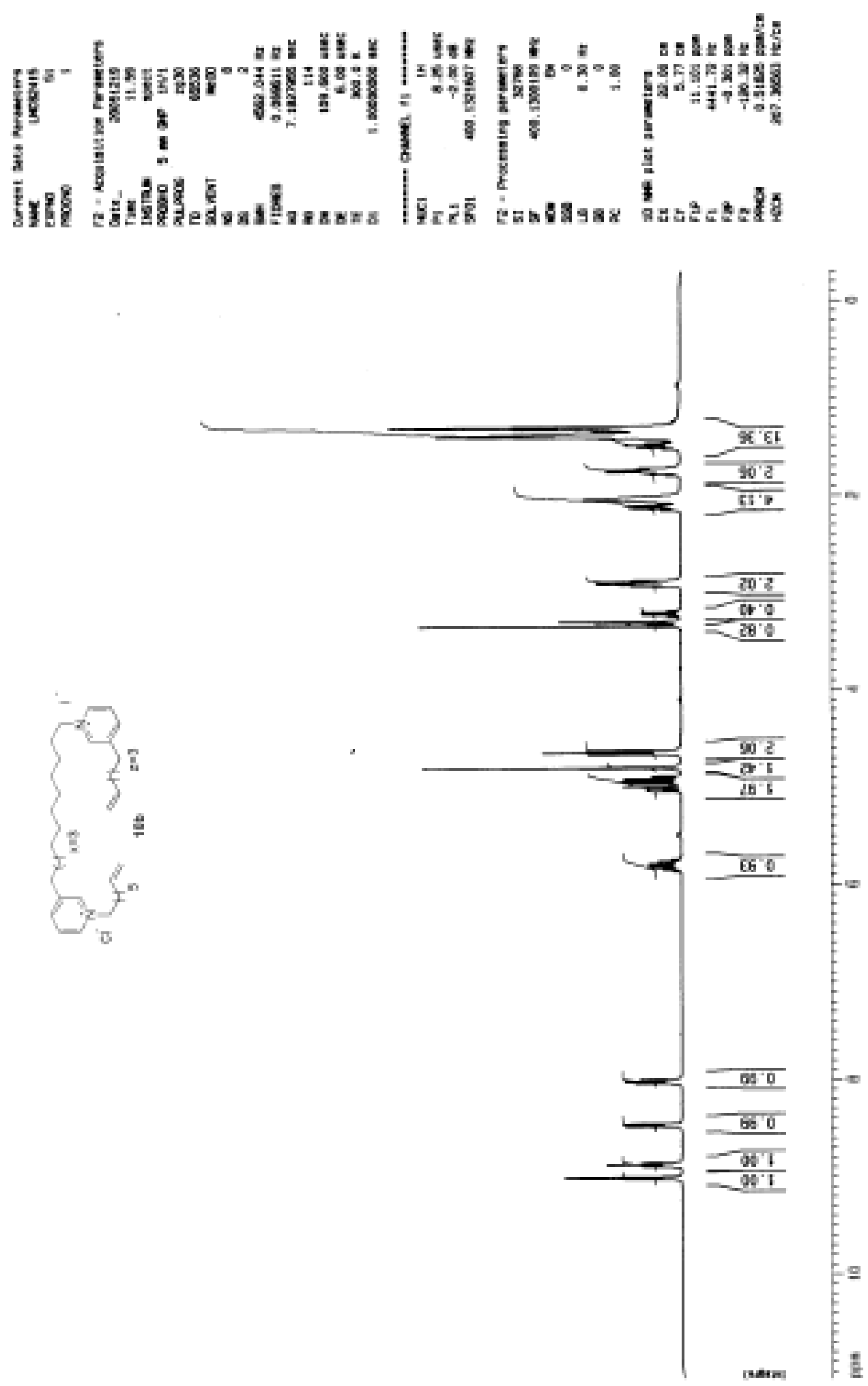

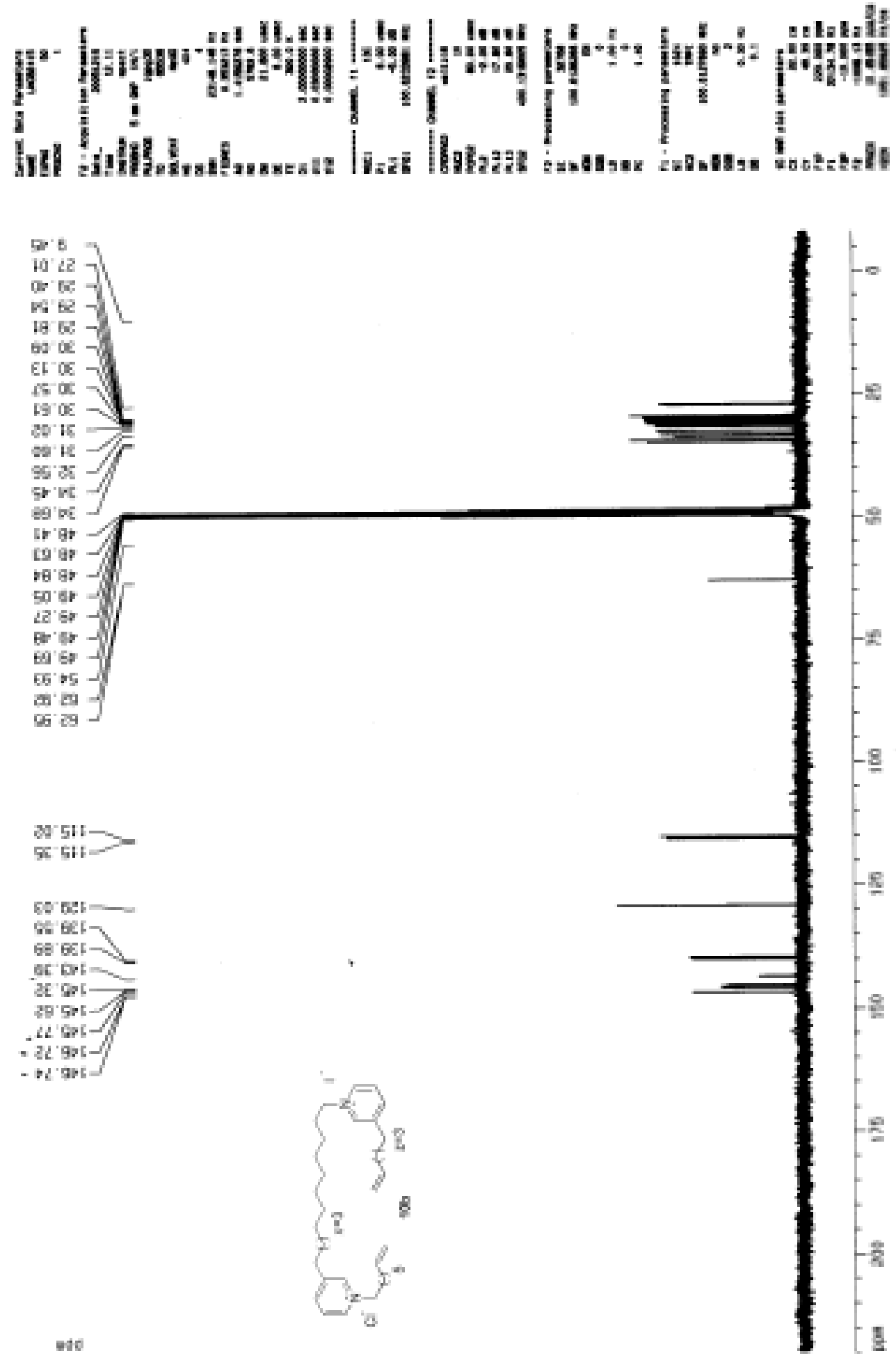

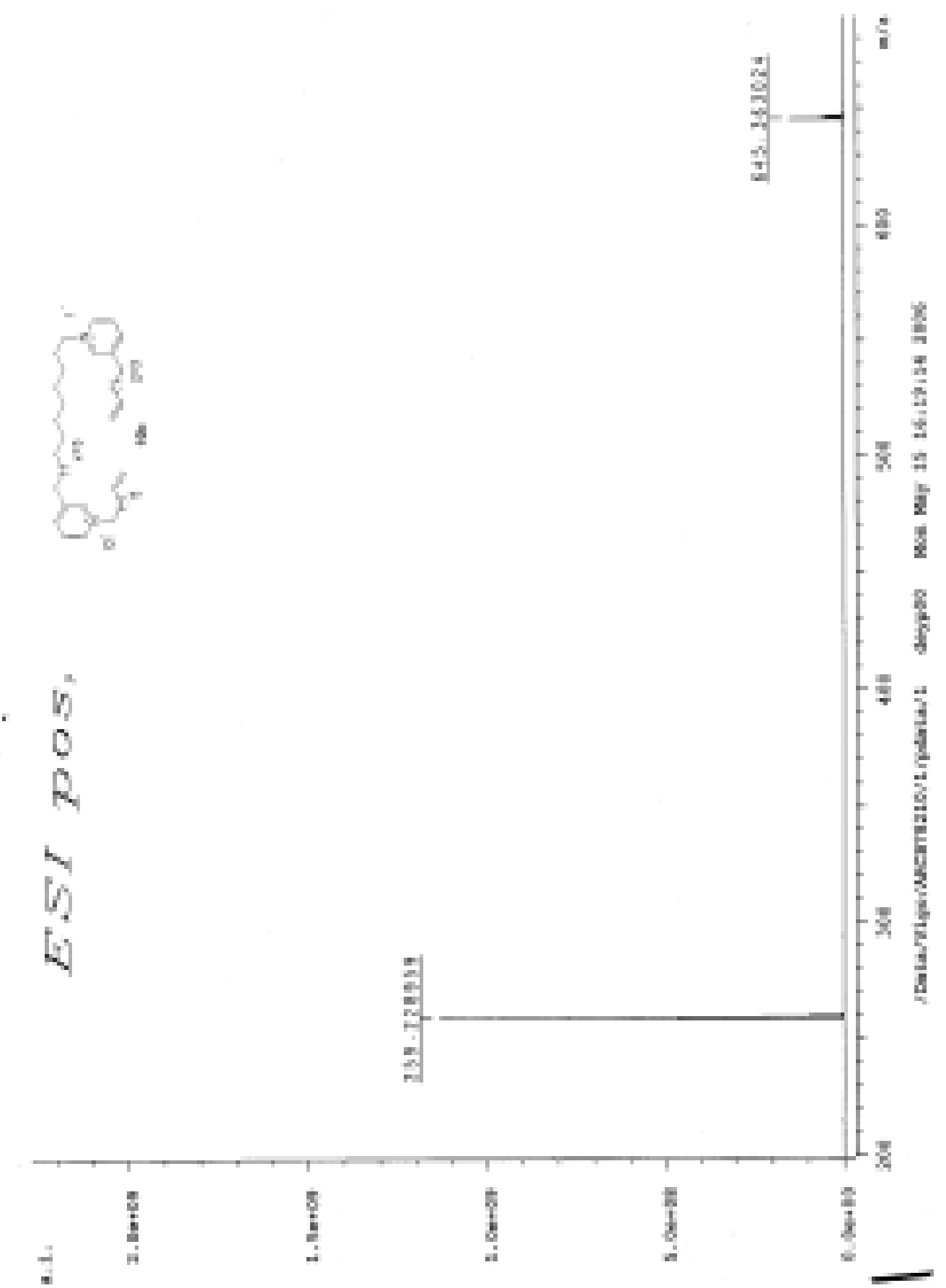


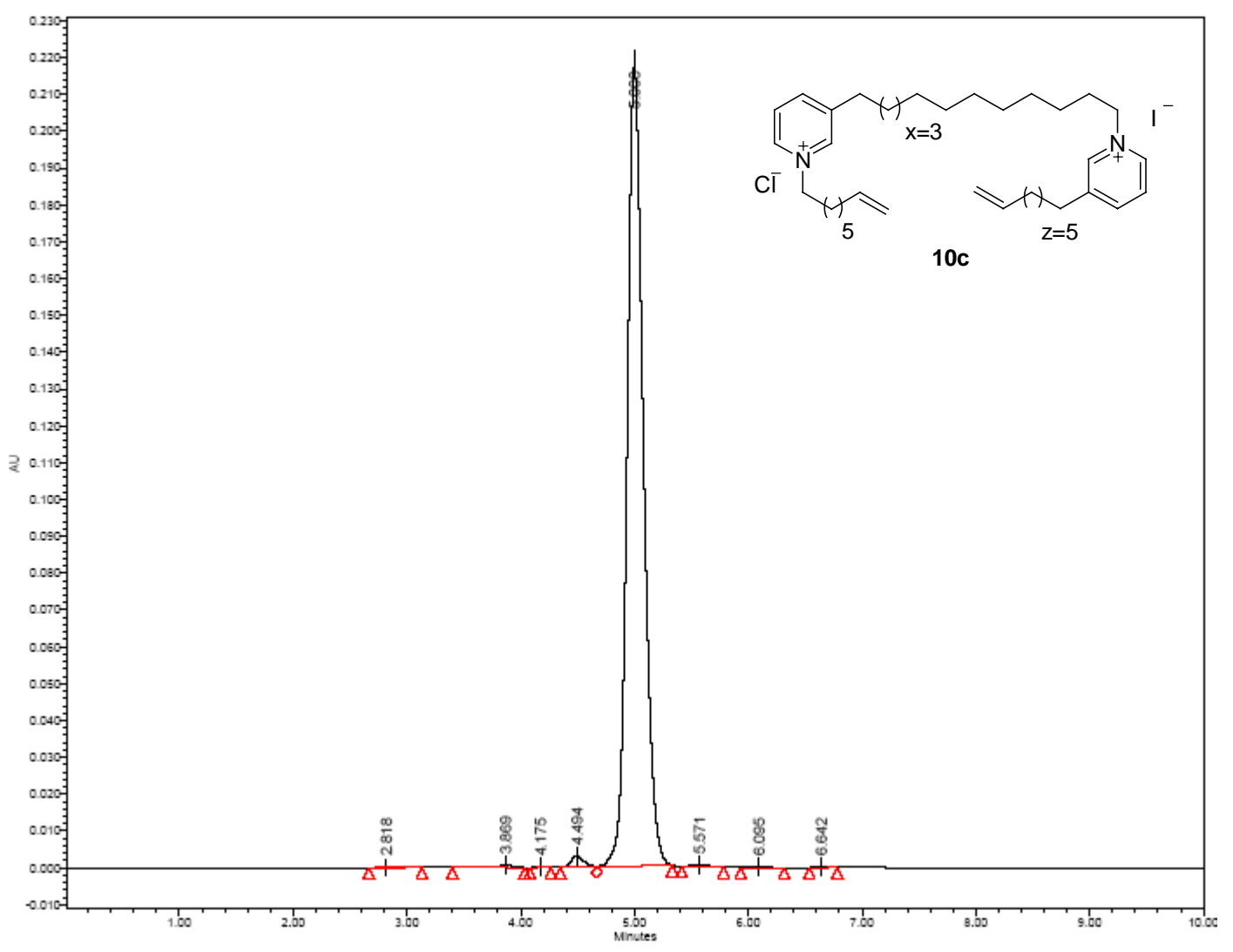

\begin{tabular}{|l|l|r|r|r|r|l|l|l|l|l|}
\hline & Name & $\begin{array}{c}\text { Retention Time } \\
(\mathrm{min})\end{array}$ & $\begin{array}{c}\text { Area } \\
\left(\mu \mathrm{V}^{2} \mathrm{sec}\right)\end{array}$ & \% Area & $\begin{array}{c}\text { Height } \\
(\mu \mathrm{V})\end{array}$ & Int Type & Amount & Units & Peak Type & Peak Codes \\
\hline 1 & & 2.818 & 959 & 0.04 & 62 & $\mathrm{BB}$ & & & Unknown & \\
\hline 2 & & 3.869 & 6715 & 0.31 & 695 & $\mathrm{BB}$ & & & Unknown & \\
\hline 3 & & 4.175 & 355 & 0.02 & 65 & $\mathrm{BB}$ & & & Unknown & \\
\hline 4 & & 4.494 & 23084 & 1.05 & 3021 & BV & & & Unknown & \\
\hline 5 & & 5.000 & 2158065 & 98.27 & 218843 & VB & & & Unknown & \\
\hline 6 & & 5.571 & 4686 & 0.21 & 405 & B B & & & Unknown & \\
\hline 7 & & 6.095 & 1321 & 0.06 & 159 & B B & & & Unknown & \\
\hline 8 & & 6.642 & 928 & 0.04 & 131 & B B & & & Unknown & \\
\hline
\end{tabular}

Develosil C-30

$\mathrm{H}_{2} 0 / \mathrm{CH}_{3} \mathrm{CN}: 17 / 83$ (0.1\% TFA)

Flow: $3.2 \mathrm{~mL} / \mathrm{min}$

$\mathrm{v}=267 \mathrm{~nm}$ 

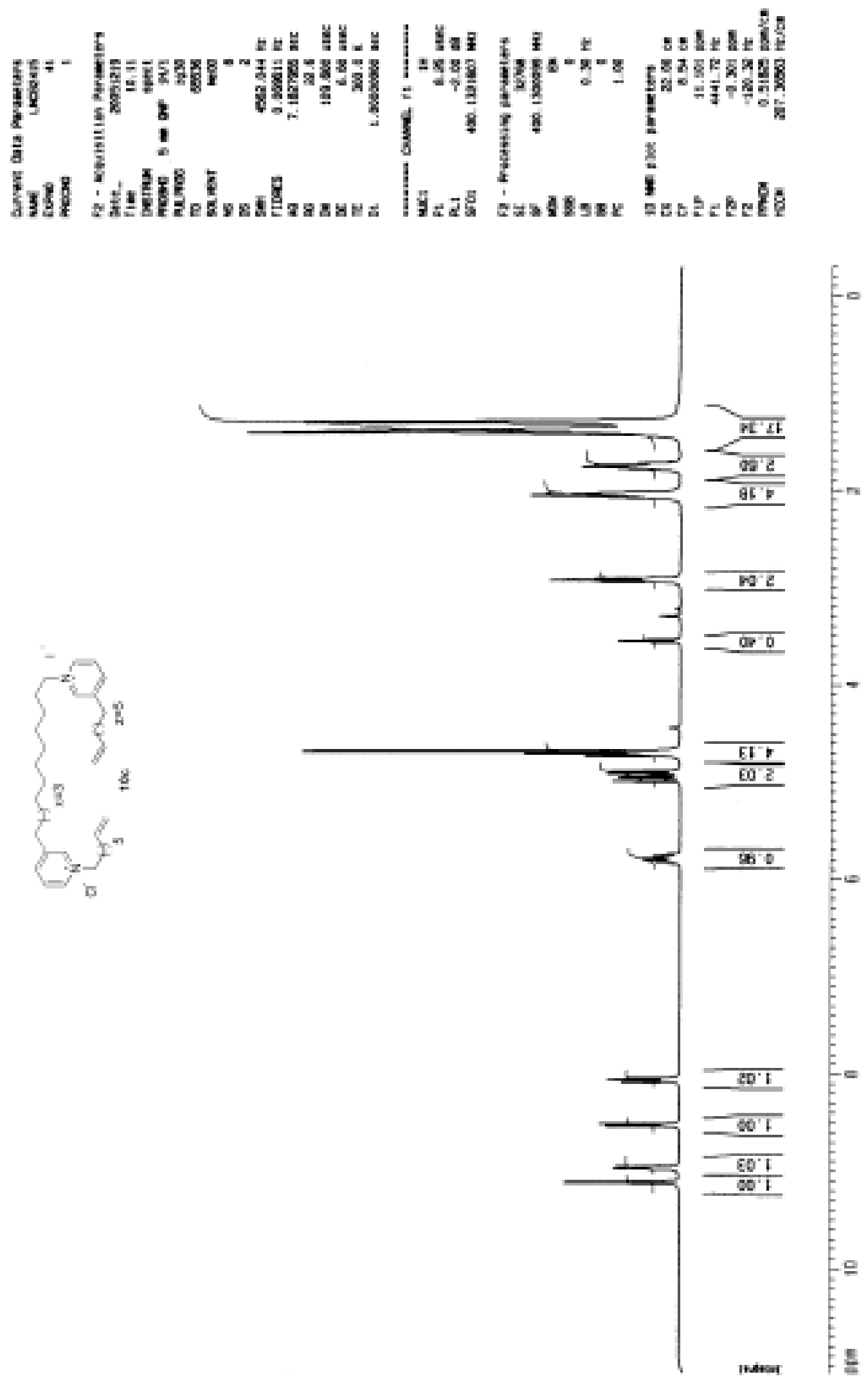

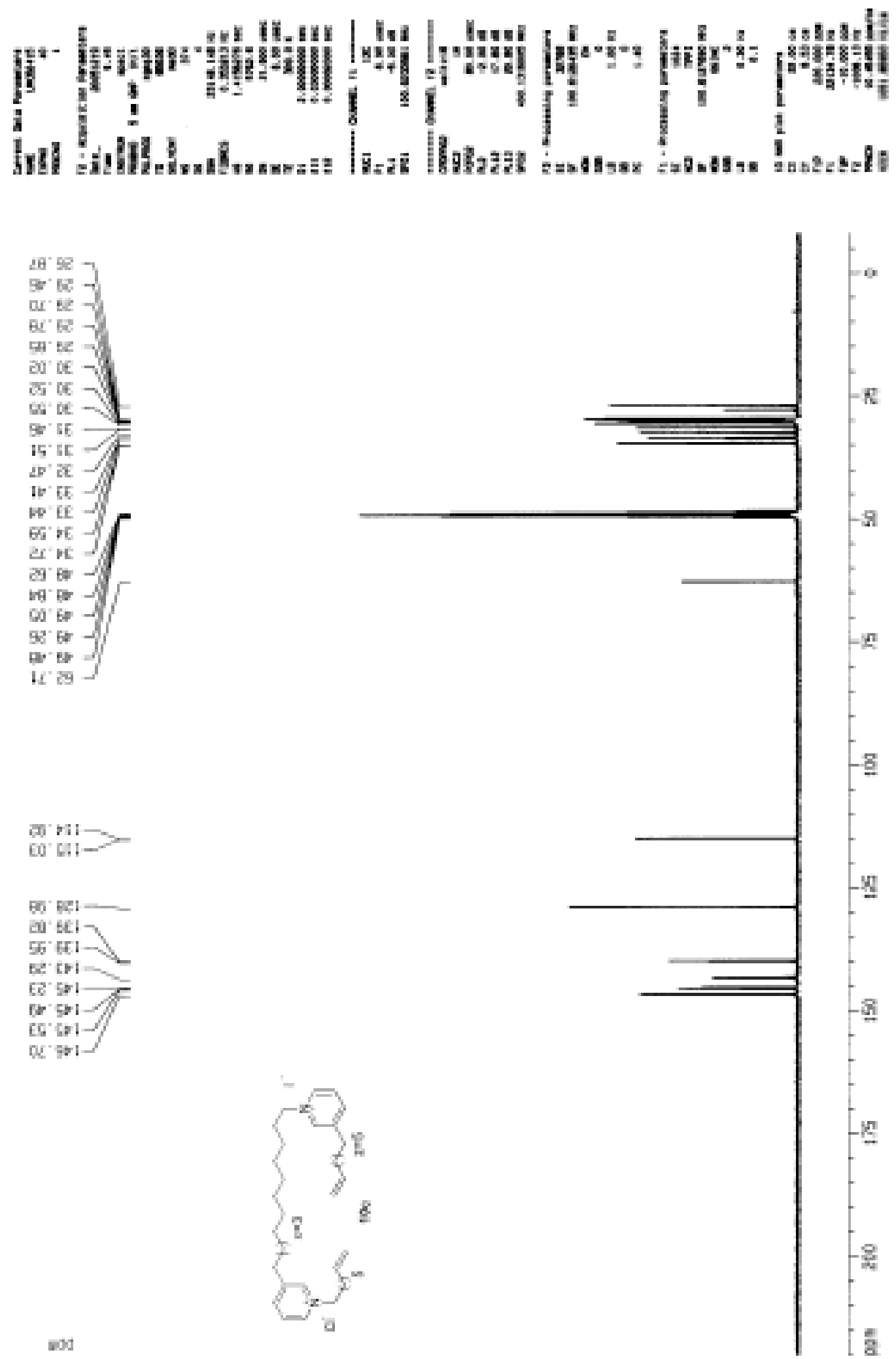

it1

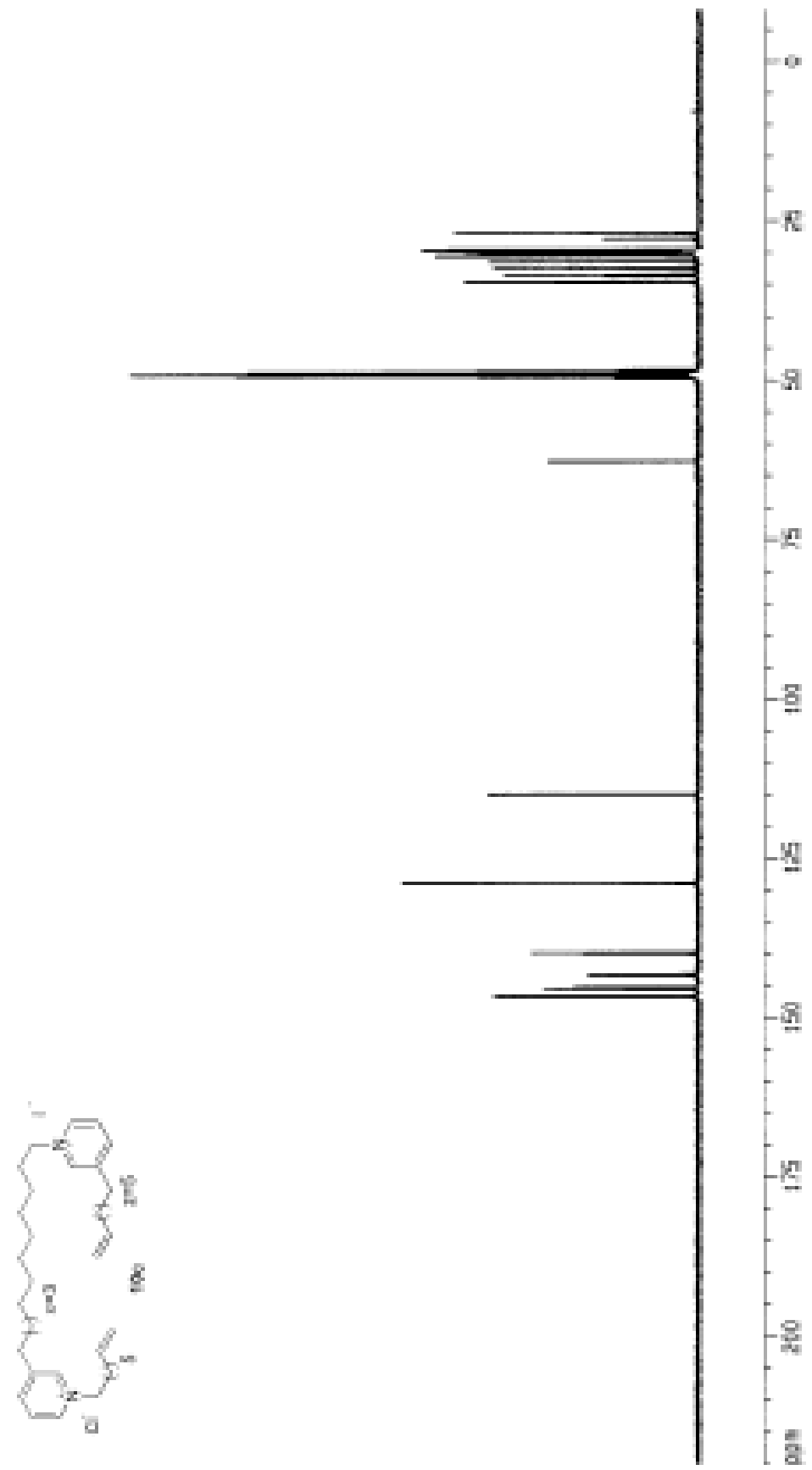

1911- $11=$

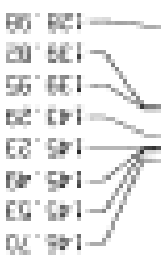



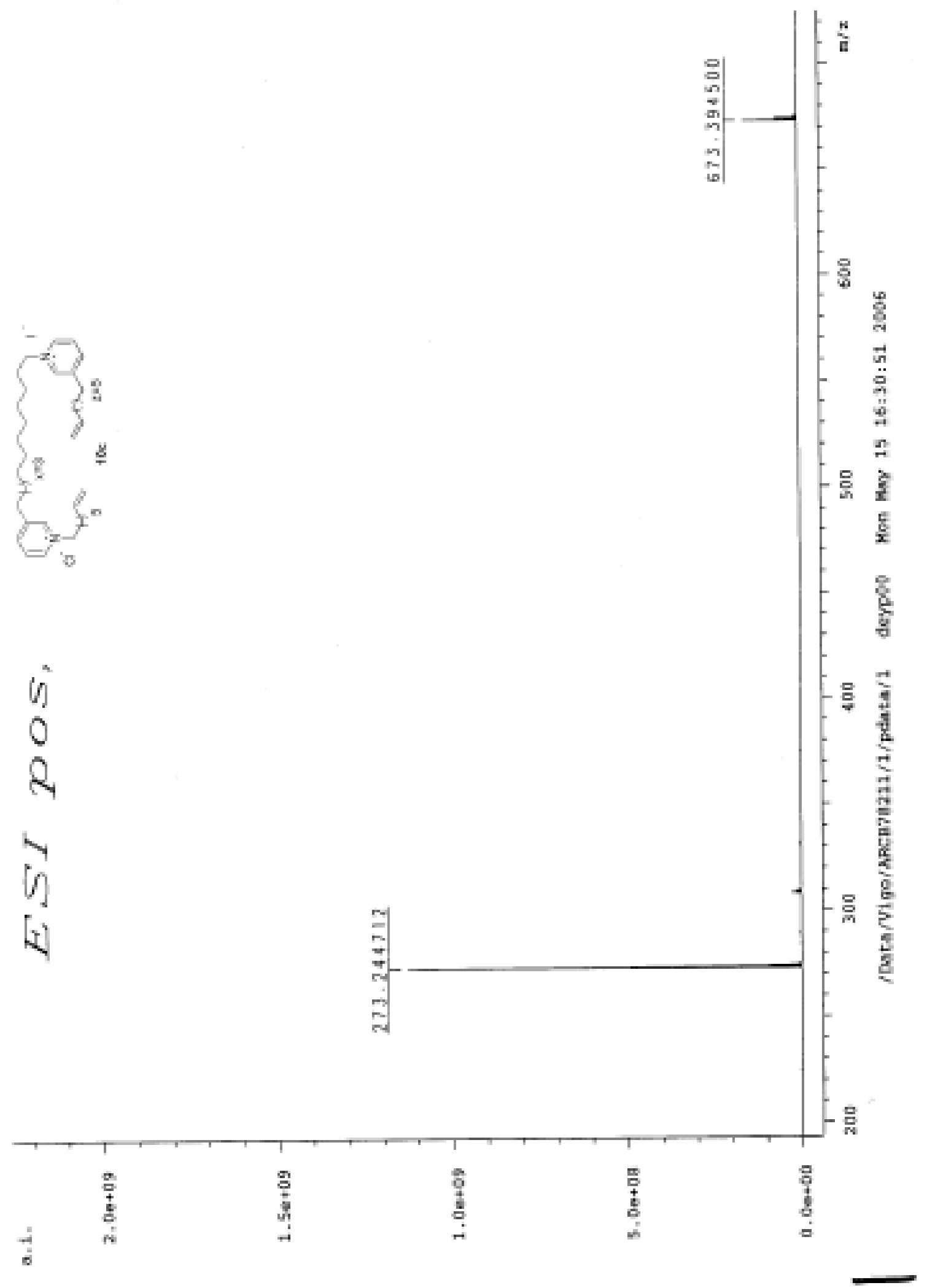


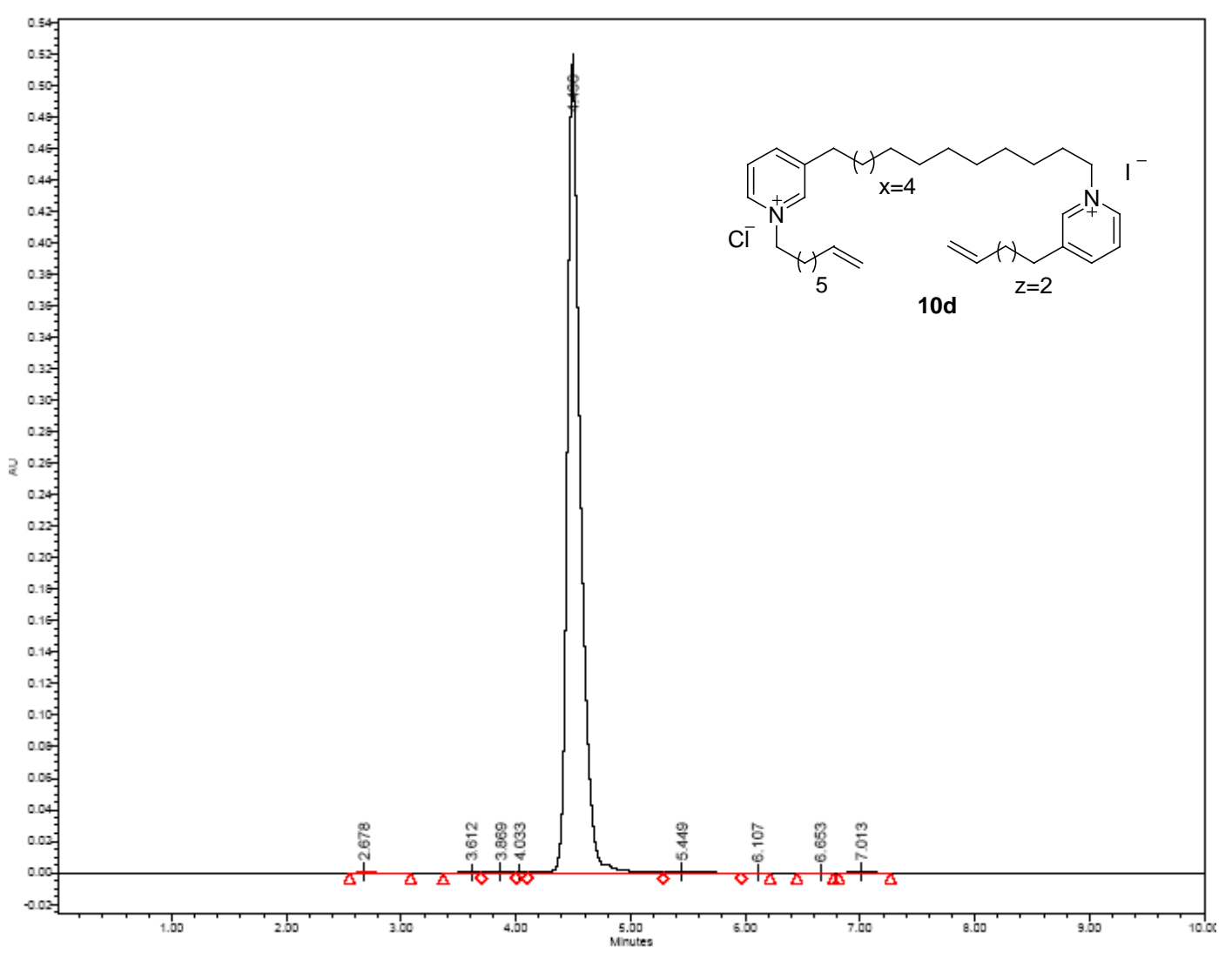

\begin{tabular}{|l|l|r|r|r|r|l|l|l|l|l|}
\hline & Name & $\begin{array}{c}\text { Retention Time } \\
(\mathrm{min})\end{array}$ & $\begin{array}{c}\text { Area } \\
\left(\mu \mathrm{V}^{*} \mathrm{sec}\right)\end{array}$ & \% Area & $\begin{array}{c}\text { Height } \\
(\mu \mathrm{V})\end{array}$ & Int Type & Amount & Units & Peak Type & Peak Codes \\
\hline 1 & & 2.678 & 3164 & 0.08 & 358 & BB & & & Unknown & \\
\hline 2 & & 3.612 & 1424 & 0.03 & 136 & BV & & & Unknown & \\
\hline 3 & & 3.869 & 6528 & 0.16 & 810 & $\mathrm{~W}$ & & & Unknown & \\
\hline 4 & & 4.033 & 685 & 0.02 & 129 & $\mathrm{~W}$ & & & Unknown & I19 \\
\hline 5 & & 4.496 & 4138283 & 99.34 & 515653 & $\mathrm{~W}$ & & & Unknown & \\
\hline 6 & & 5.449 & 7733 & 0.19 & 441 & $\mathrm{~W}$ & & & Unknown & \\
\hline 7 & & 6.107 & 425 & 0.01 & 52 & VB & & & Unknown & \\
\hline 8 & & 6.653 & 563 & 0.01 & 80 & B B & & & Unknown & \\
\hline 9 & & 7.013 & 6867 & 0.16 & 726 & BB & & & Unknown & \\
\hline
\end{tabular}

Develosil C-30

$\mathrm{H}_{2} 0 / \mathrm{CH}_{3} \mathrm{CN}: 17 / 83$ (0.1\% TFA)

Flow: $3.2 \mathrm{~mL} / \mathrm{min}$

$v=267 \mathrm{~nm}$ 

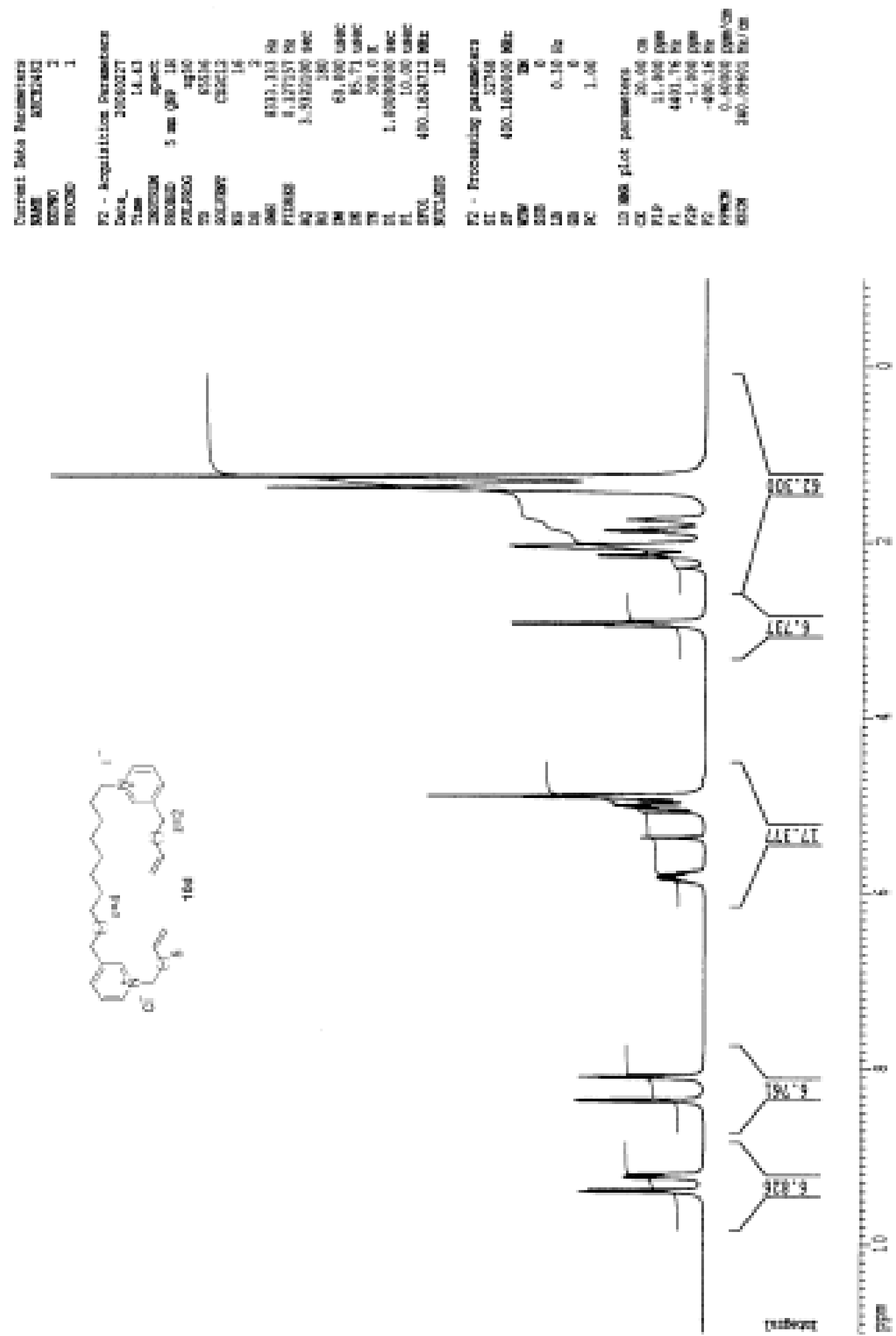


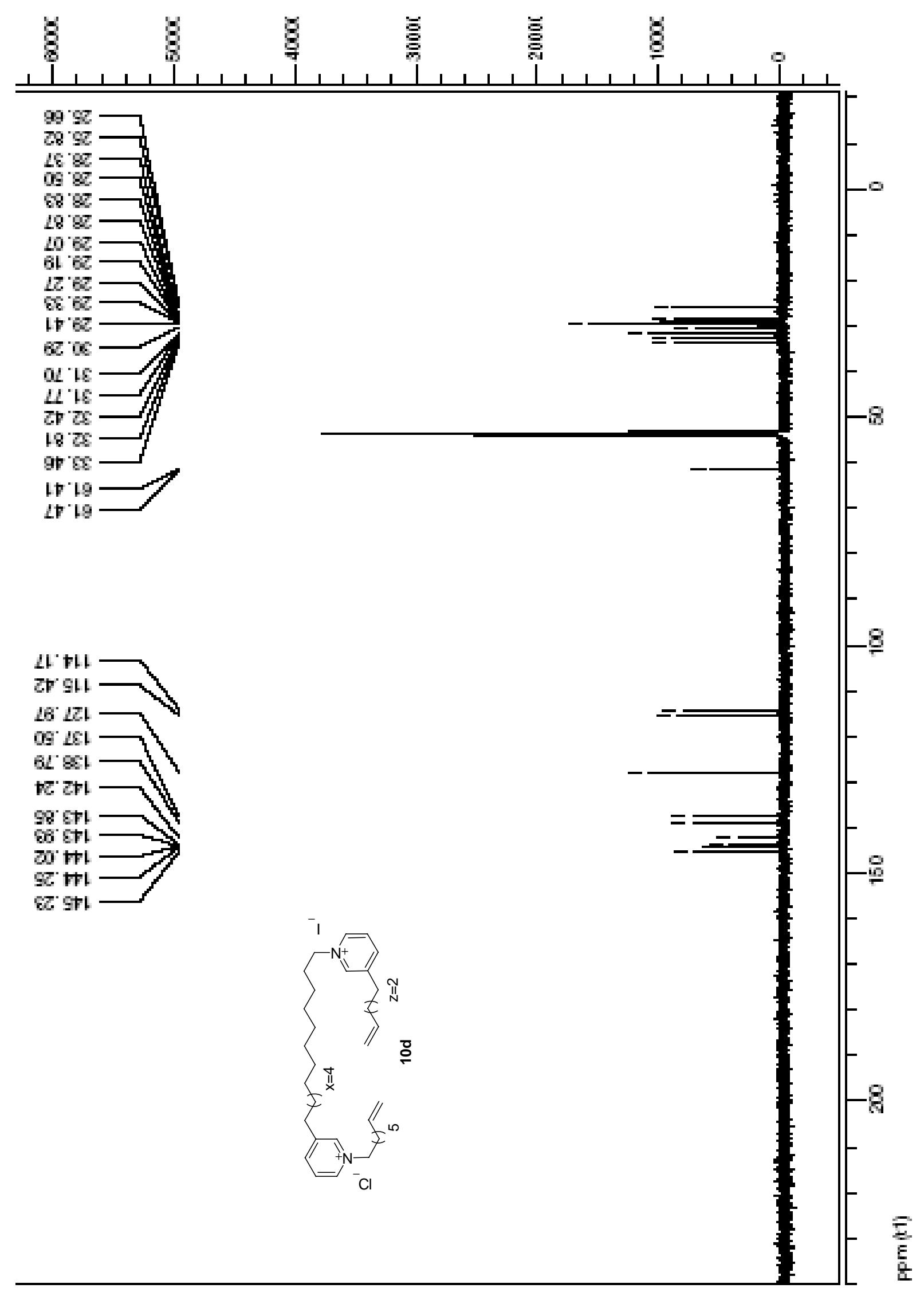




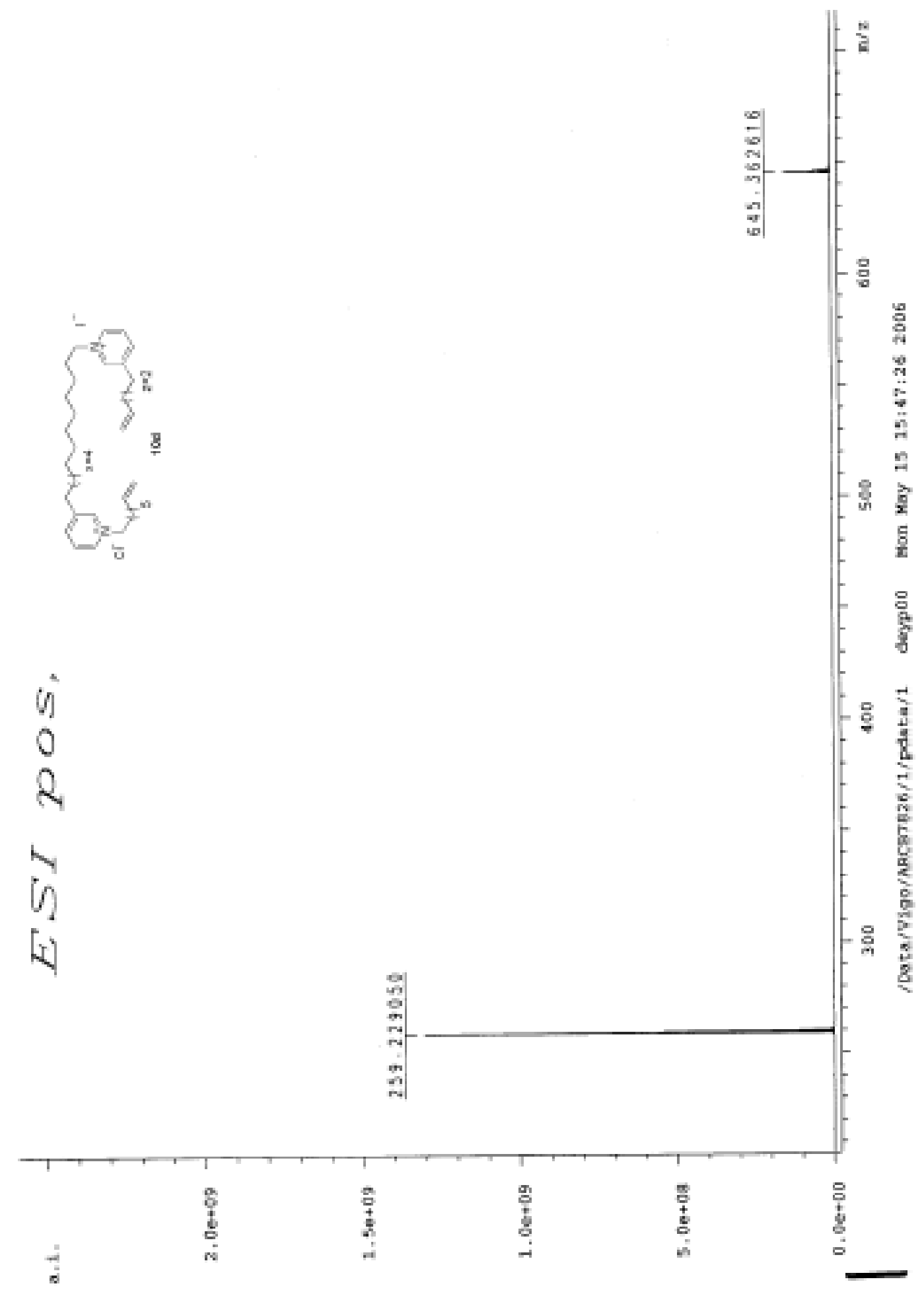




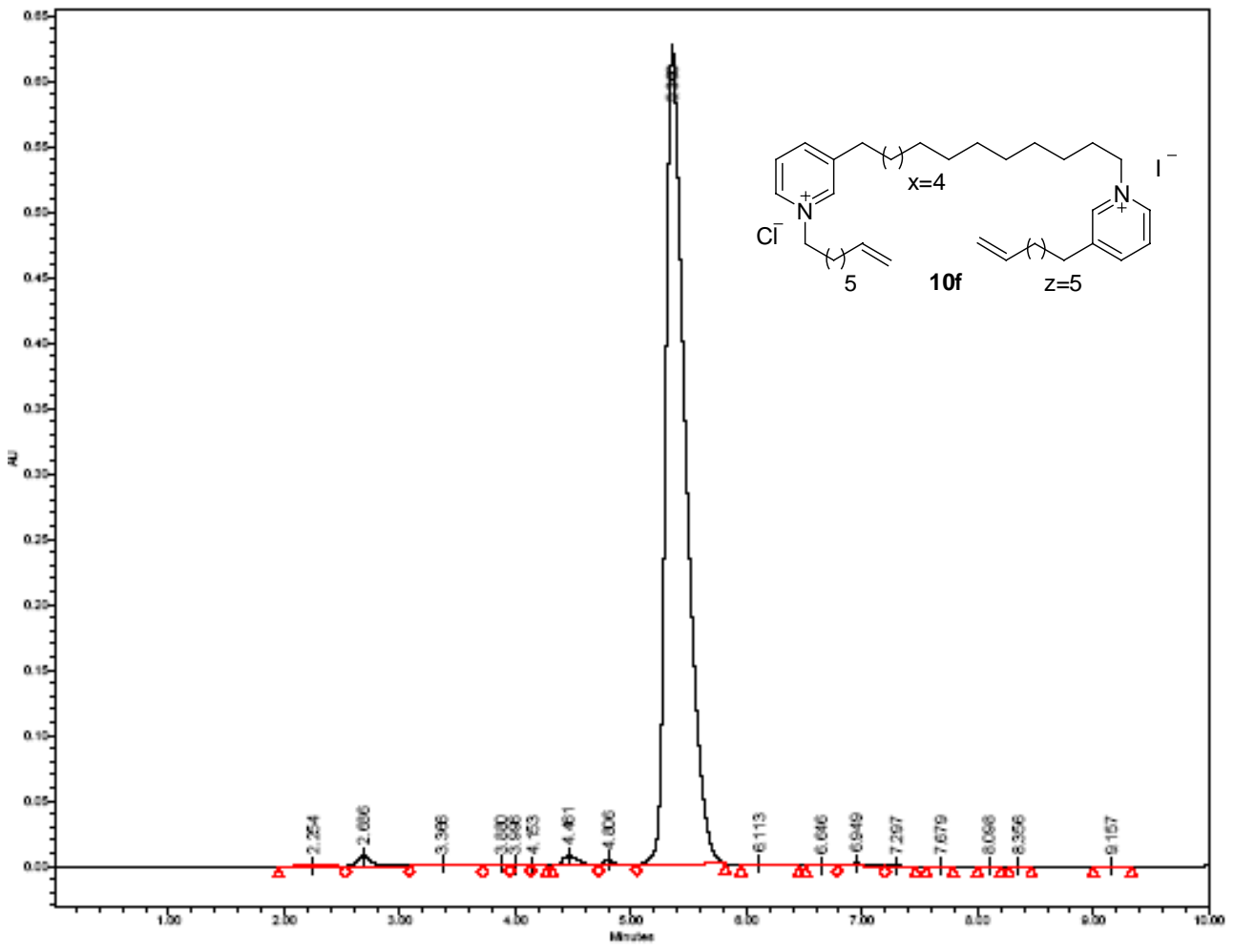

\begin{tabular}{|l|l|r|r|r|r|l|l|l|l|l|}
\hline & Name & $\begin{array}{r}\text { Retention Time } \\
(\mathrm{min})\end{array}$ & $\begin{array}{c}\text { Area } \\
\left(\mu \mathrm{V}^{\prime} \mathrm{sec}\right)\end{array}$ & $\%$ Area & $\begin{array}{c}\text { Height } \\
(\mu \mathrm{V})\end{array}$ & Int Type & Amount & Units & Peak Type & Peak Codes \\
\hline 1 & & 2.254 & 10347 & 0.13 & 711 & BV & & & Unknown & \\
\hline 2 & & 2.686 & 65421 & 0.85 & 7619 & VV & & & Unknown & \\
\hline 3 & & 3.386 & 28442 & 0.34 & 1069 & VV & & & Unknown & \\
\hline 4 & & 3.880 & 9274 & 0.12 & 977 & VV & & & Unknown & \\
\hline 5 & & 3.996 & 5414 & 0.07 & 873 & VV & & & Unknown & \\
\hline 6 & & 4.153 & 495 & 0.01 & 91 & VB & & & Unknown & \\
\hline 7 & & 4.461 & 68571 & 0.90 & 7078 & BV & & & Unknown & \\
\hline 8 & & 4.806 & 26058 & 0.34 & 3625 & VV & & & Unknown & \\
\hline 9 & & 5.360 & 7488265 & 96.78 & 628273 & VB & & & Unknown & \\
\hline 10 & & 6.113 & 12209 & 0.16 & 880 & BB & & & Unknown & \\
\hline 11 & & 6.646 & 4336 & 0.06 & 586 & BV & & & Unknown & \\
\hline 12 & & 6.949 & 17785 & 0.23 & 1901 & VV & & & Unknown & \\
\hline 13 & & 7.297 & 417 & 0.01 & 54 & VB & & & Unknown & \\
\hline 14 & & 7.679 & 288 & 0.00 & 41 & BB & & & Unknown & \\
\hline 15 & & 8.098 & 196 & 0.00 & 27 & BB & & & Unknown & \\
\hline 16 & & 8.356 & 181 & 0.00 & 25 & BB & & & Unknown & \\
\hline 17 & & 9.157 & 369 & 0.00 & 37 & BB & & & Unknown & \\
\hline
\end{tabular}

Develosil C-30

$\mathrm{H}_{2} \mathrm{O} / \mathrm{CH}_{3} \mathrm{CN}: 17 / 83$ (0.1\% TFA)

Flow: $3.2 \mathrm{~mL} / \mathrm{min}$

$\mathrm{v}=267 \mathrm{~nm}$ 

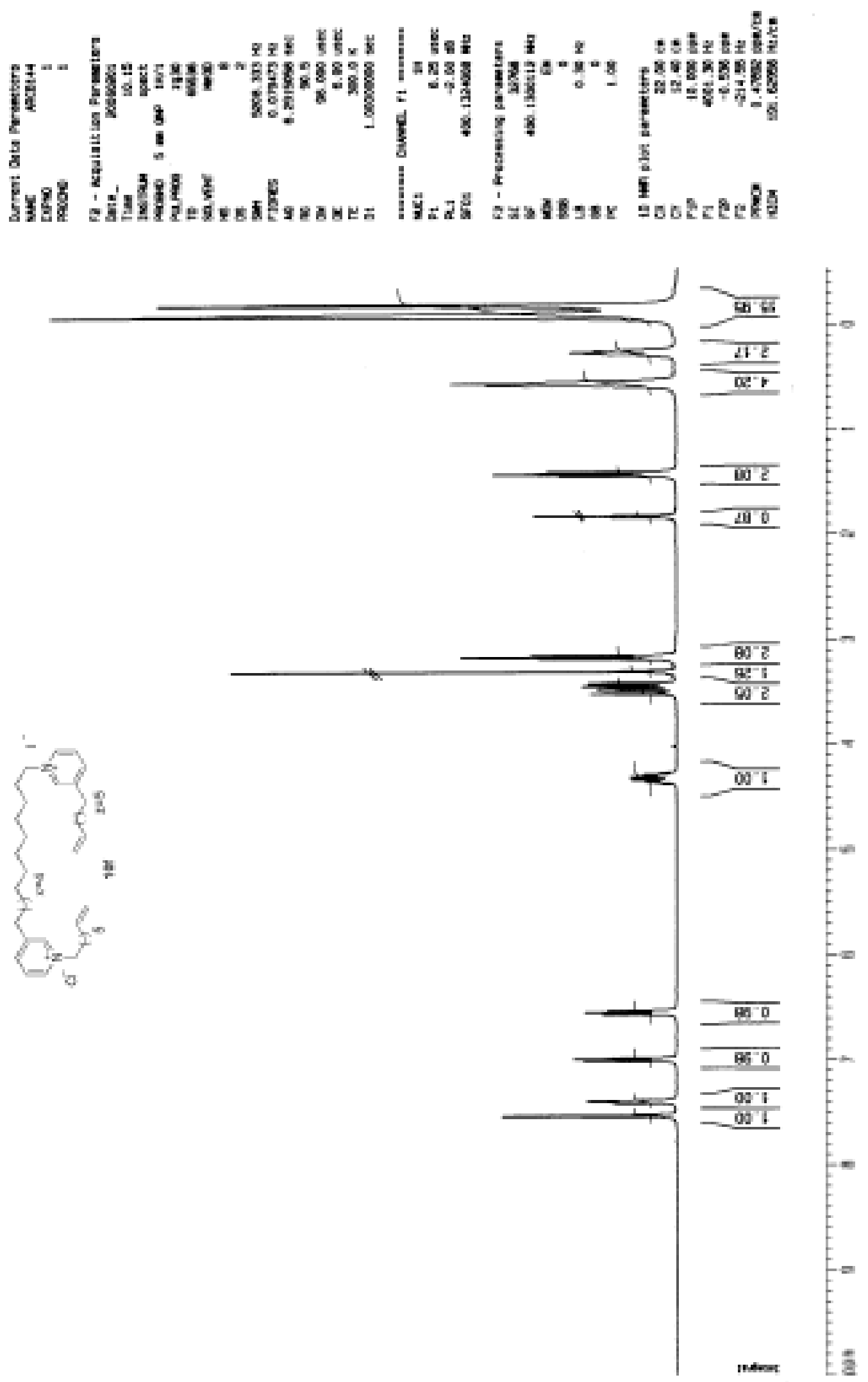

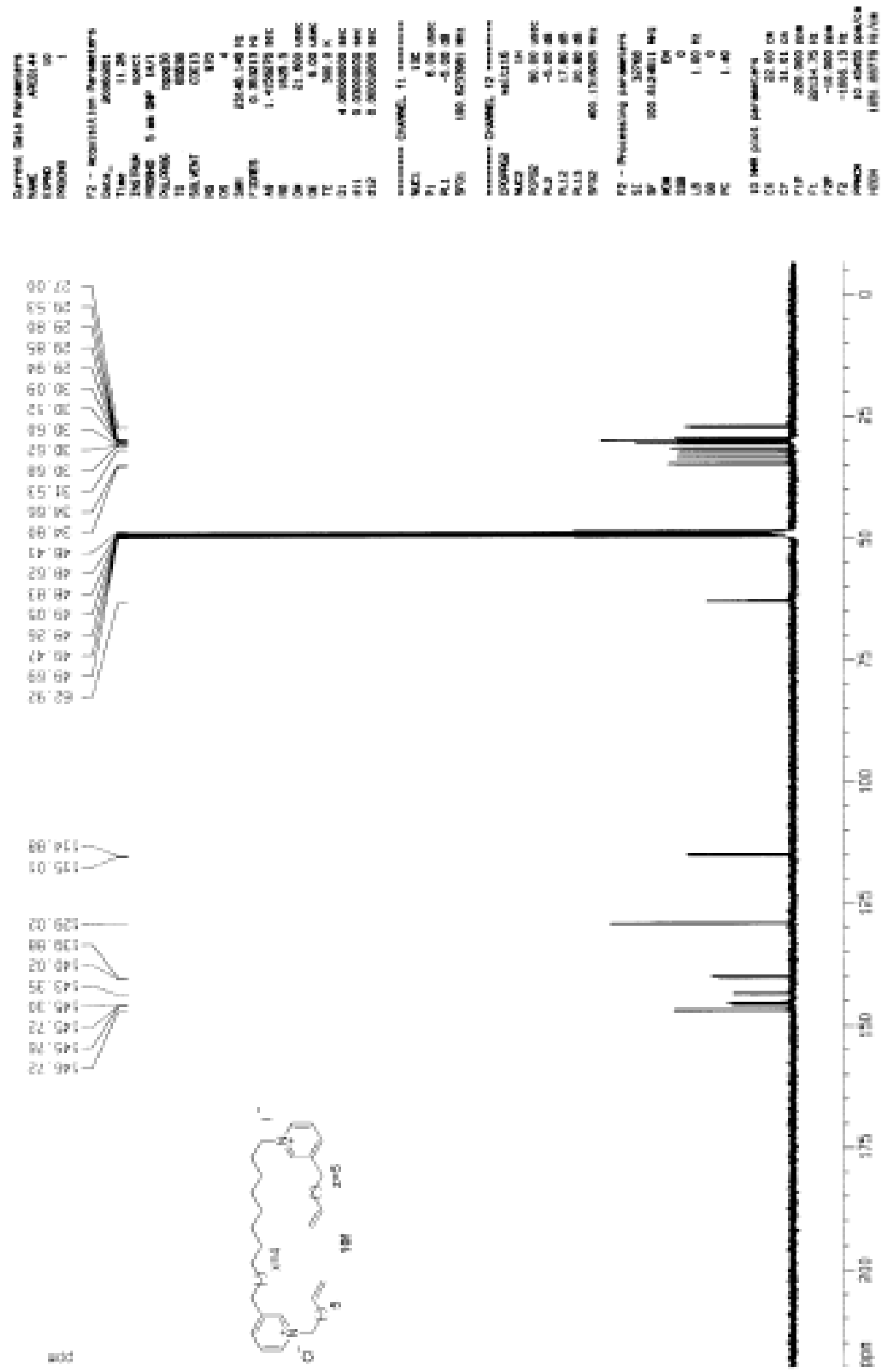


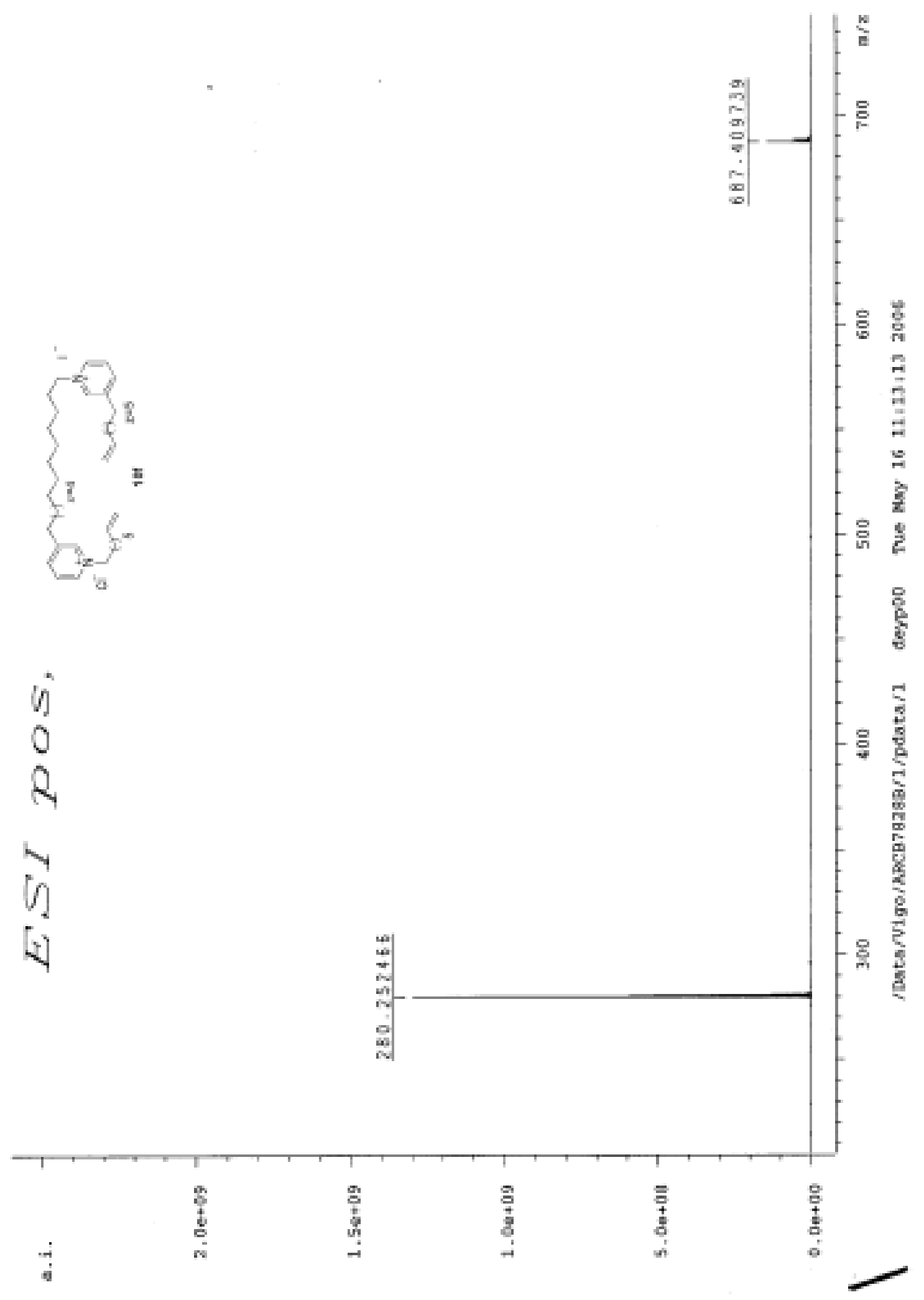




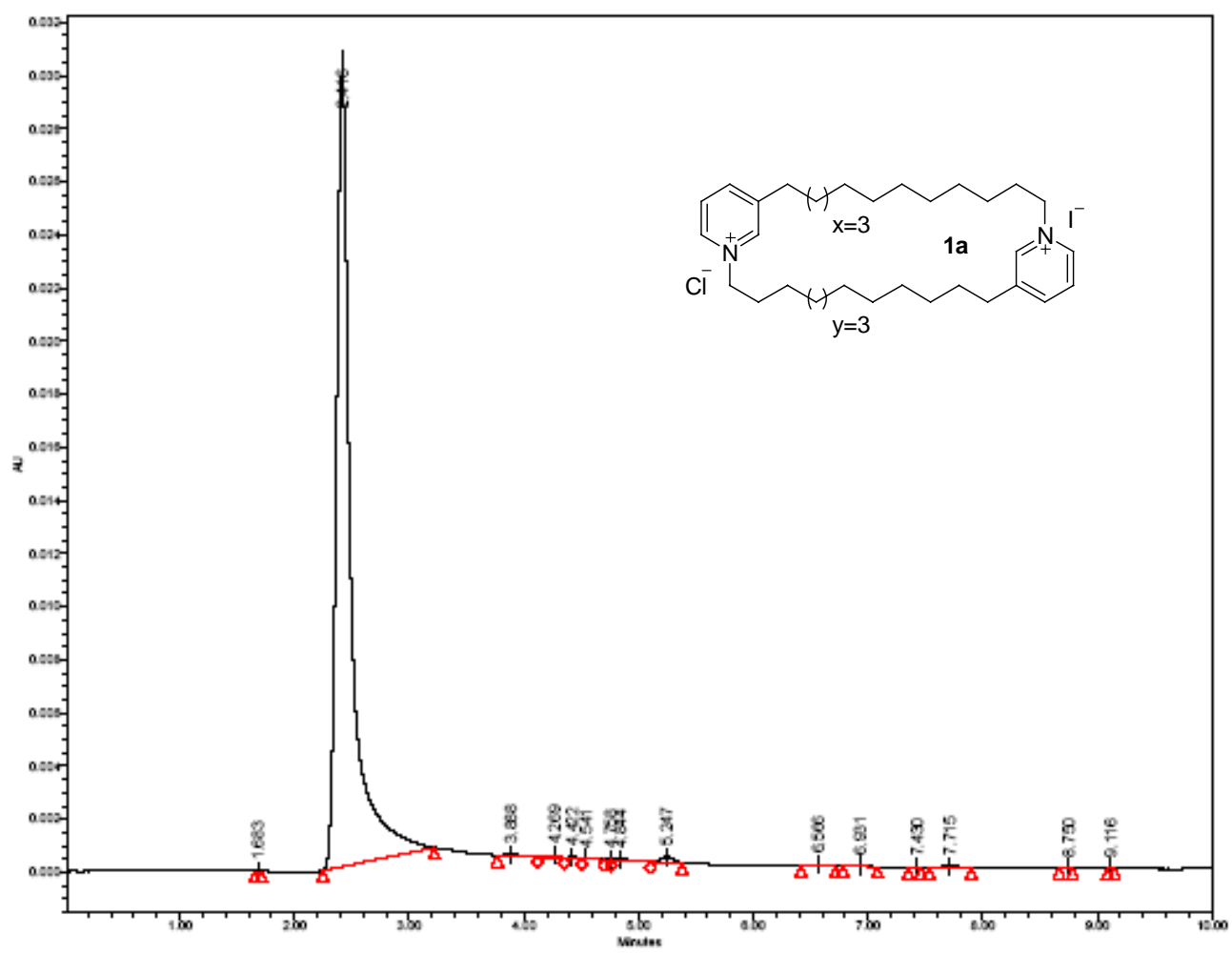

\begin{tabular}{|c|c|c|c|c|c|c|c|c|c|c|}
\hline & Name & $\begin{array}{c}\text { Retention Time } \\
\text { (min) }\end{array}$ & $\begin{array}{c}\text { Area } \\
\left(\mu V^{x} \text { sec }\right)\end{array}$ & $\%$ Area & $\begin{array}{c}\text { Height } \\
(\mu \mathrm{V})\end{array}$ & Int Type & Amount & Units & Peak Type & Peak Codes \\
\hline 1 & & 1.683 & 17 & 0.01 & 8 & BB & & & Unknown & 119 \\
\hline 2 & & 2.416 & 270483 & 97.89 & 30294 & BB & & & Unknown & \\
\hline 3 & & 3.888 & 670 & 0.24 & 70 & BV & & & Unknown & \\
\hline 4 & & 4.269 & 702 & 0.25 & 96 & W & & & Unknown & \\
\hline 5 & & 4.422 & 382 & 0.14 & 68 & w & & & Unknown & \\
\hline 6 & & 4.541 & 277 & 0.10 & 28 & $\mathrm{w}$ & & & Unknown & I19 \\
\hline 7 & & 4.758 & 113 & 0.04 & 38 & w & & & Unknown & I19 I38 \\
\hline 8 & & 4.844 & 690 & 0.25 & 54 & w & & & Unknown & \\
\hline 8 & & 5.247 & 1611 & 0.58 & 223 & VB & & & Unknown & \\
\hline 10 & & 6.568 & 233 & 0.08 & 28 & BB & & & Unknown & \\
\hline 11 & & 6.931 & 296 & 0.11 & 29 & BB & & & Unknown & \\
\hline 12 & & 7.430 & 30 & 0.01 & 9 & BB & & & Unknown & I19 \\
\hline 13 & & 7.715 & 759 & 0.27 & 74 & BB & & & Unknown & \\
\hline 14 & & 8.750 & 21 & 0.01 & 8 & BB & & & Unknown & 119 \\
\hline 15 & & 9.116 & 15 & 0.01 & 8 & BB & & & Unknown & 119 \\
\hline
\end{tabular}

\section{Develosil C-30}

$\mathrm{H}_{2} \mathrm{O} / \mathrm{MeOH}: 7 / 93$ (0.1\% TFA)

Flow: $3.2 \mathrm{~mL} / \mathrm{min}$

$\mathrm{v}=267 \mathrm{~nm}$ 

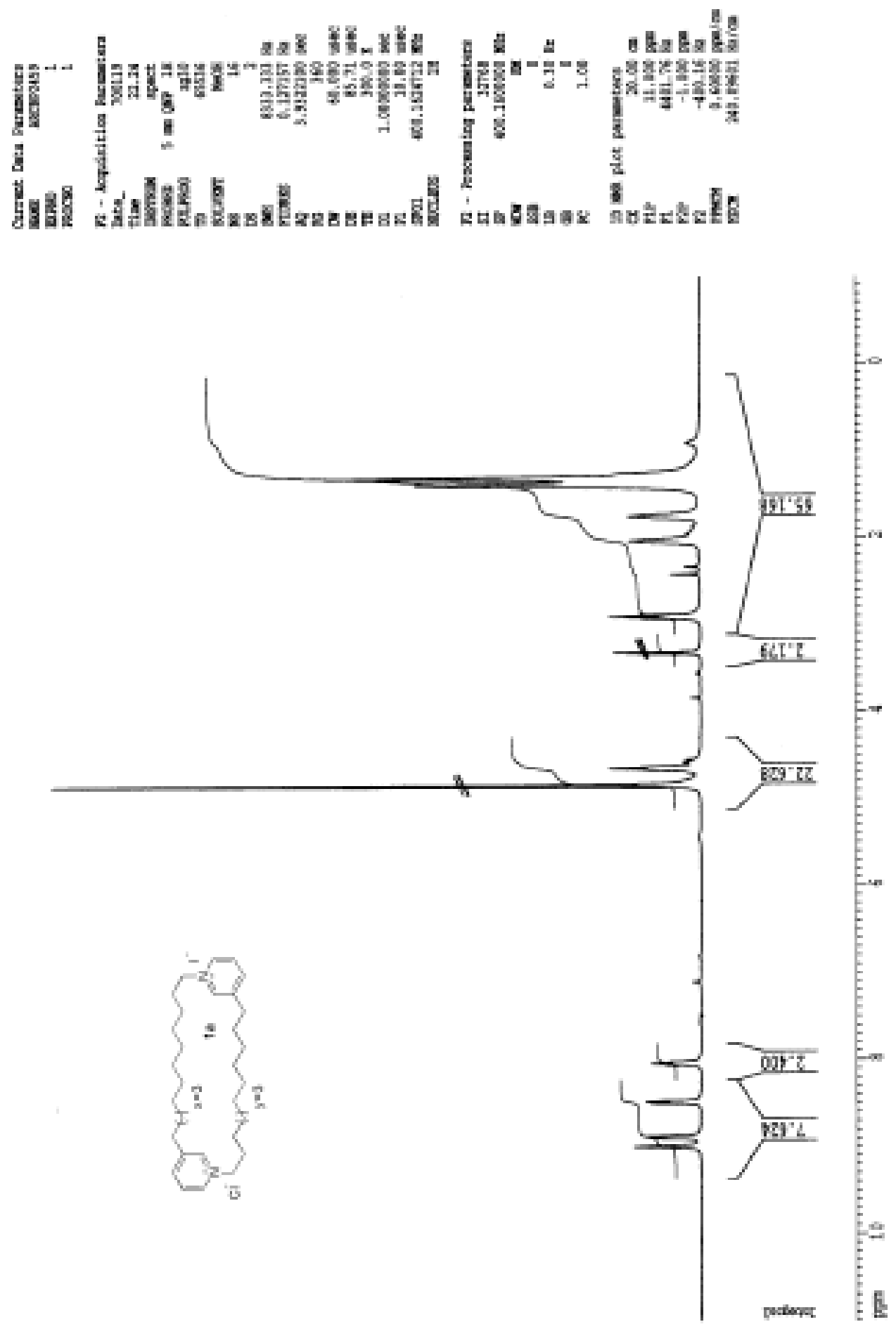

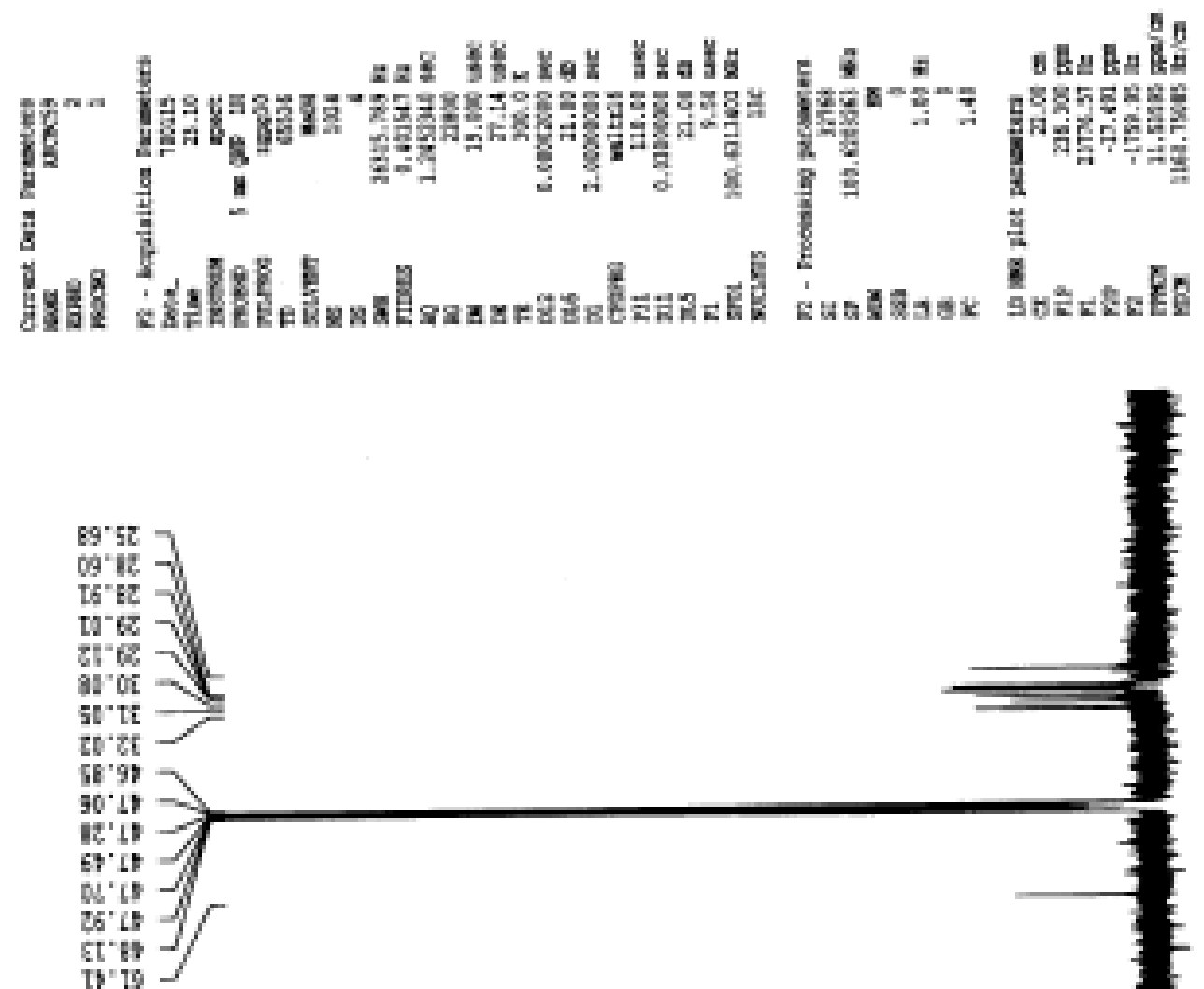

LFLL-
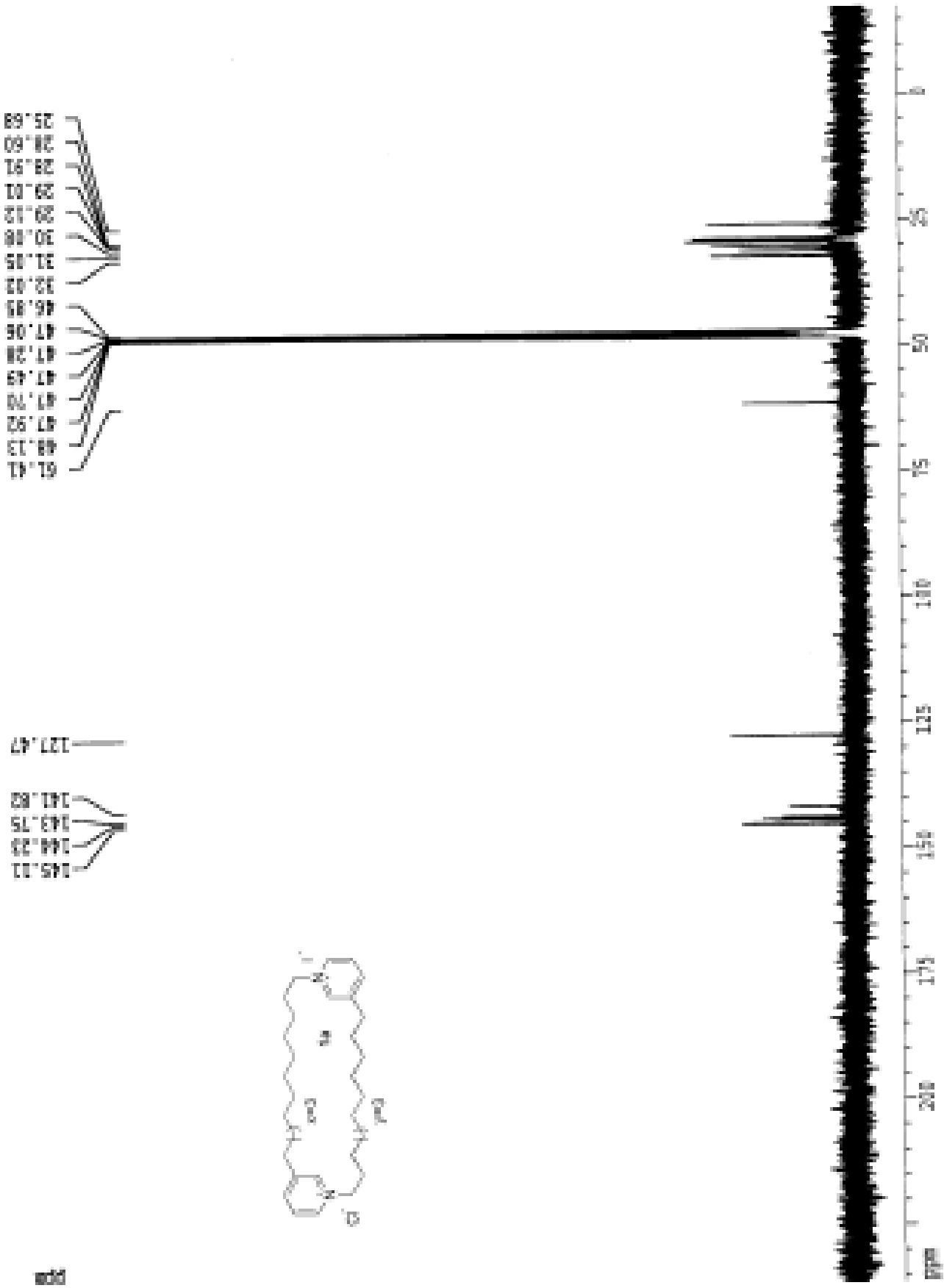

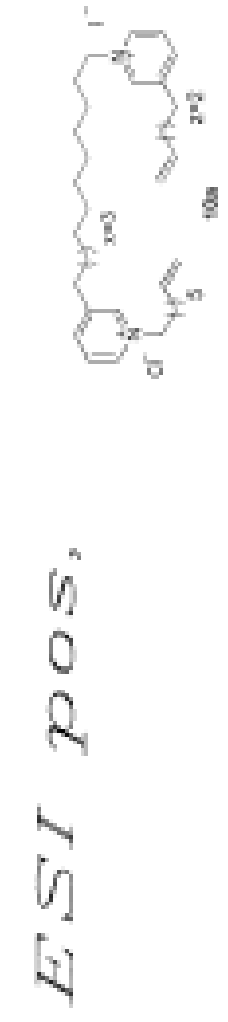

in

2

is

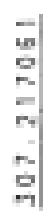

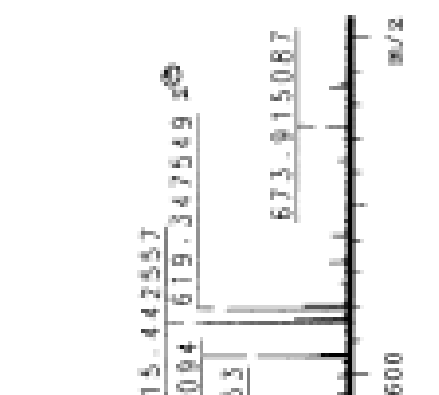

.

당

함

$\approx$
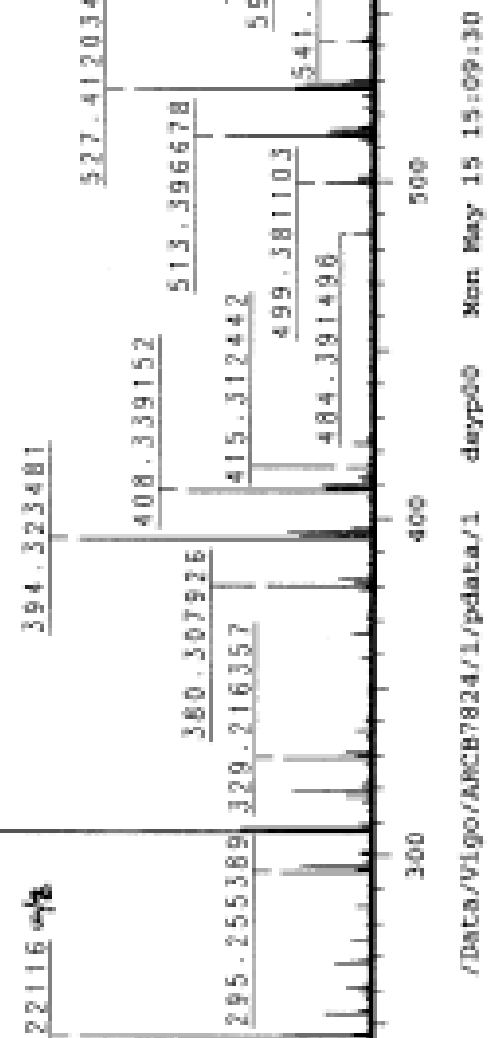

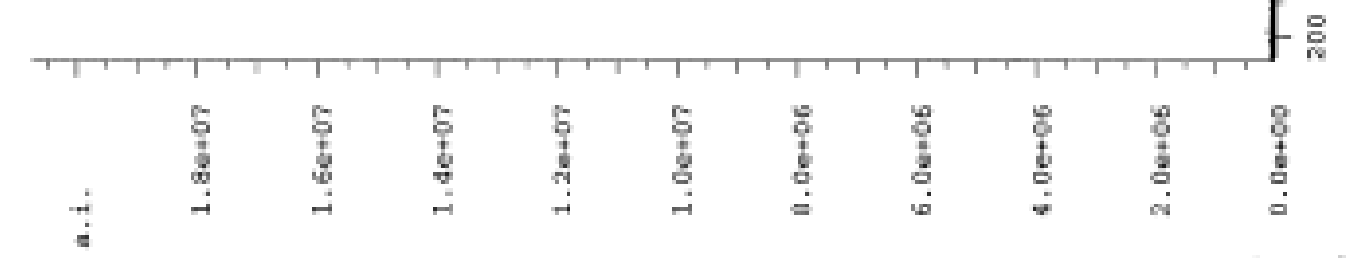




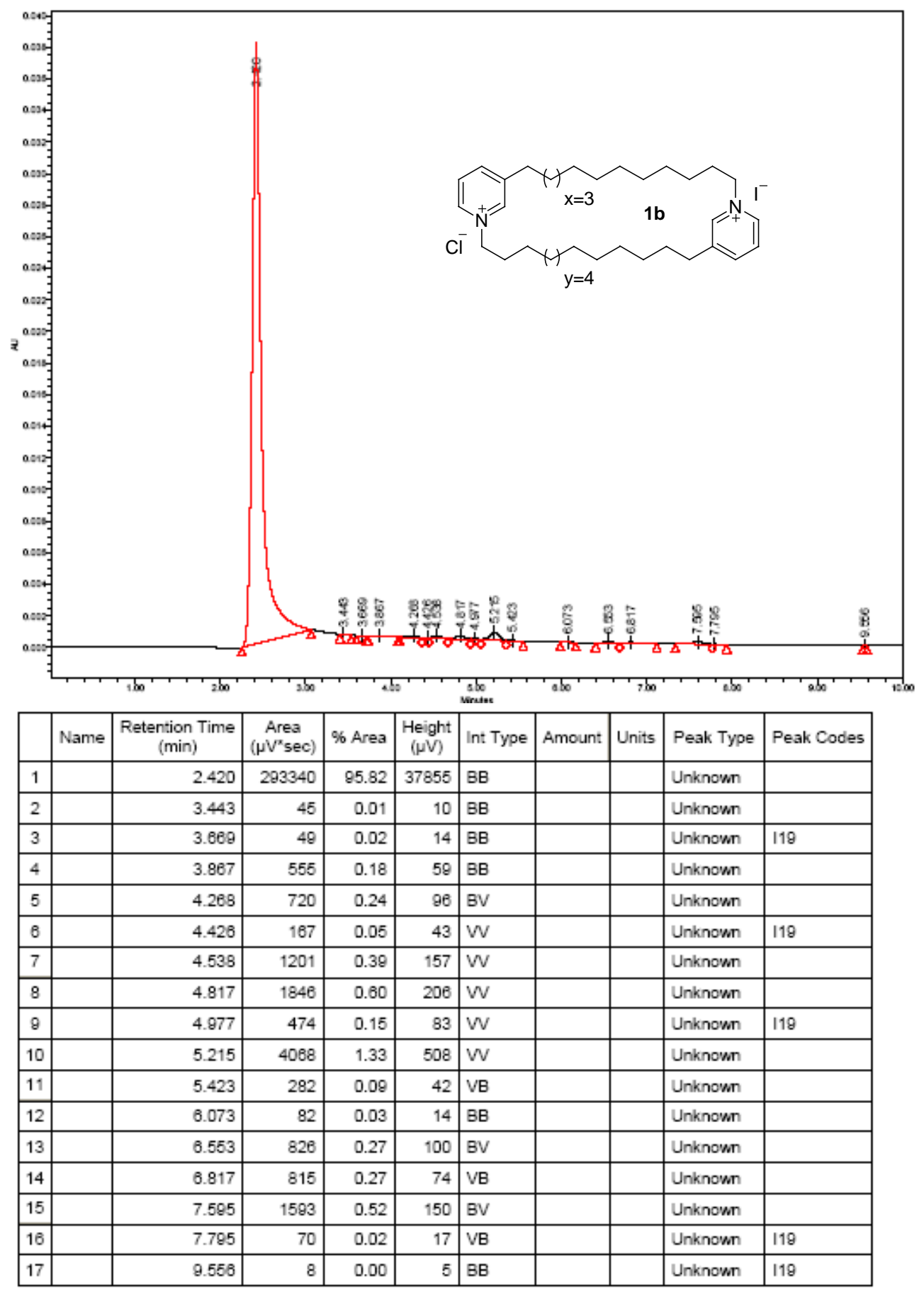

Develosil C-30

$\mathrm{H}_{2} 0 / \mathrm{MeOH}: 7 / 93$ (0.1\% TFA)

Flow: $3.2 \mathrm{~mL} / \mathrm{min}$

$\mathrm{v}=267 \mathrm{~nm}$ 

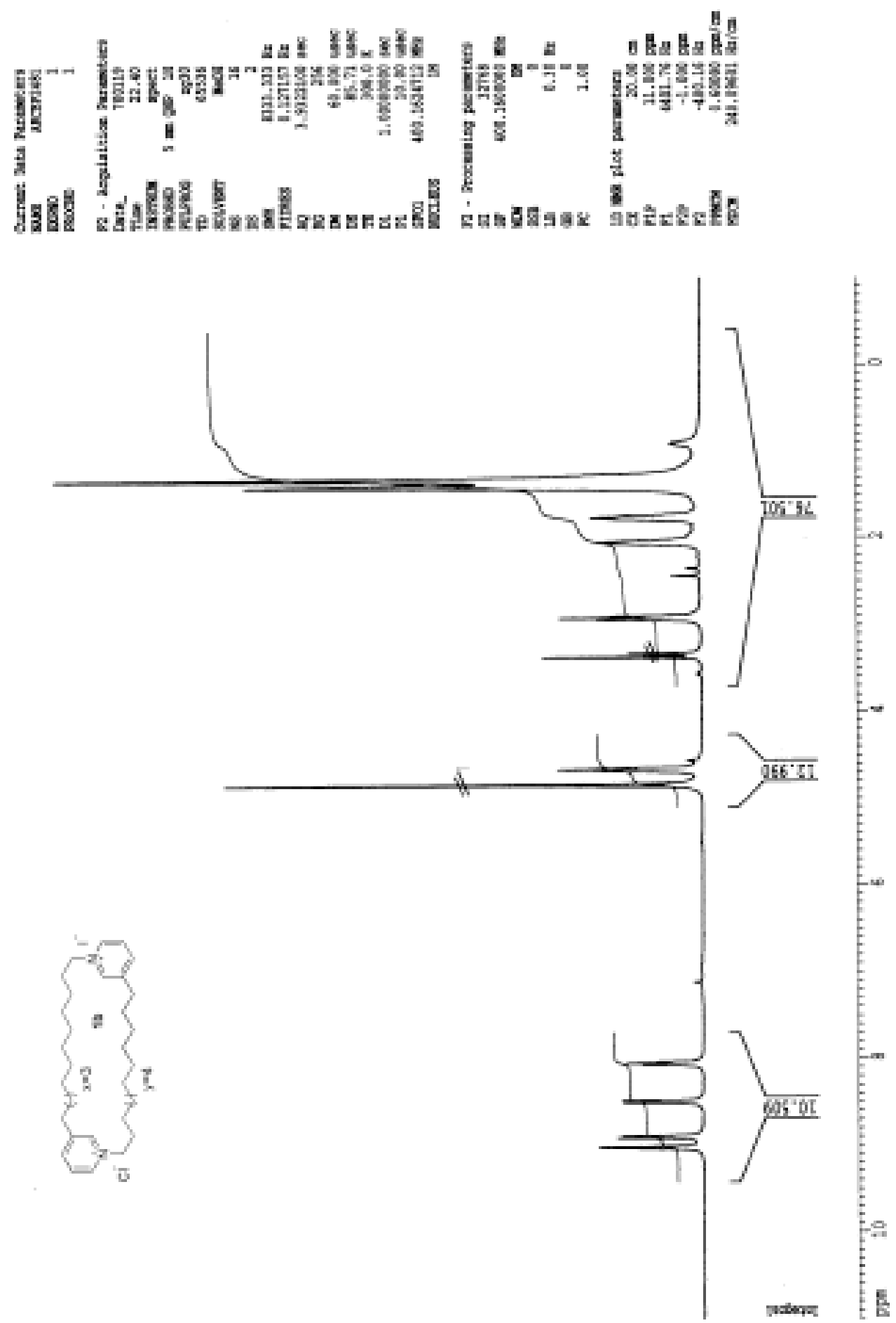

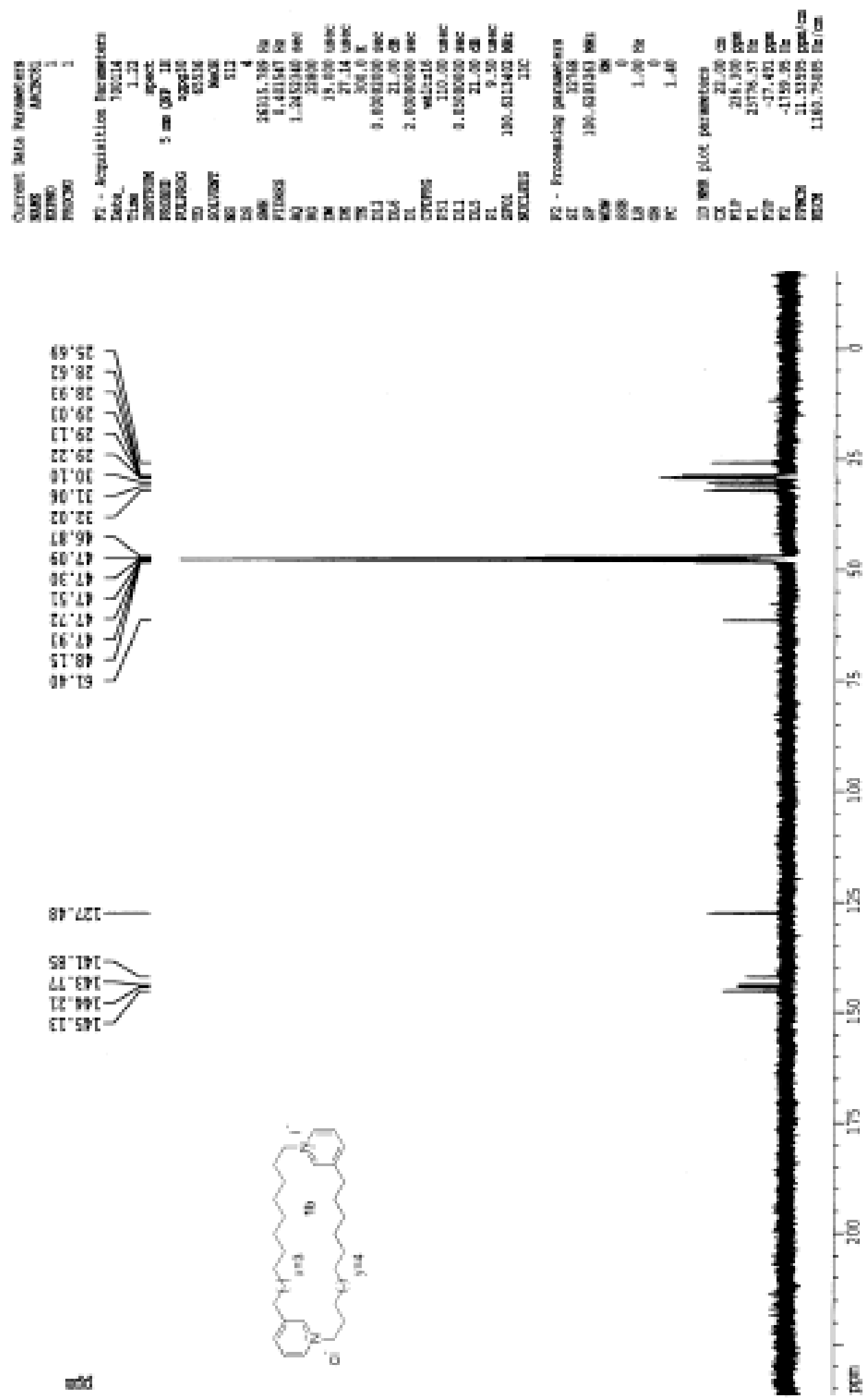

$8 t^{\prime} u t$

รม"17:

LI' Ert-

L" $m[-$

हt 5 t

atd 

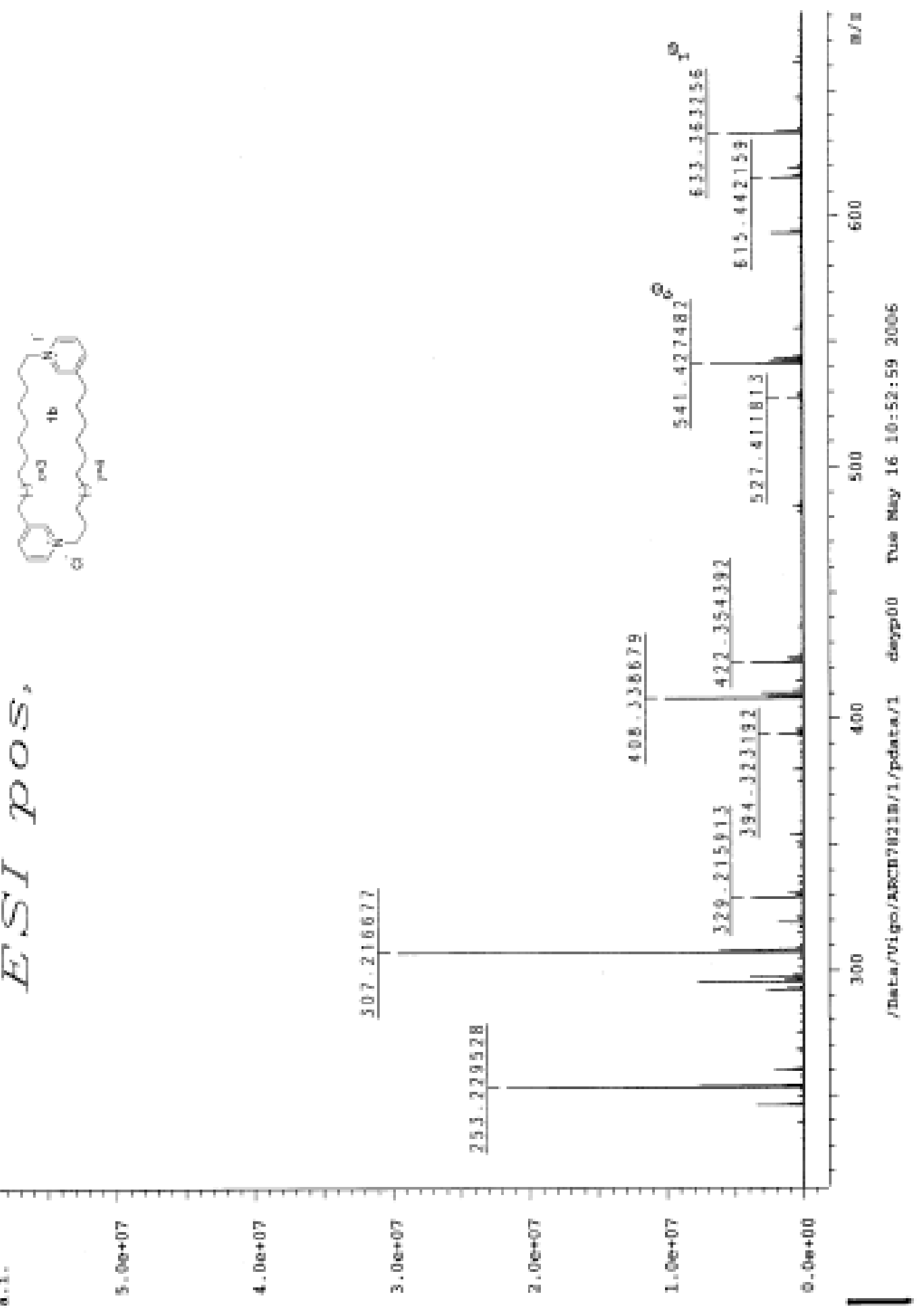


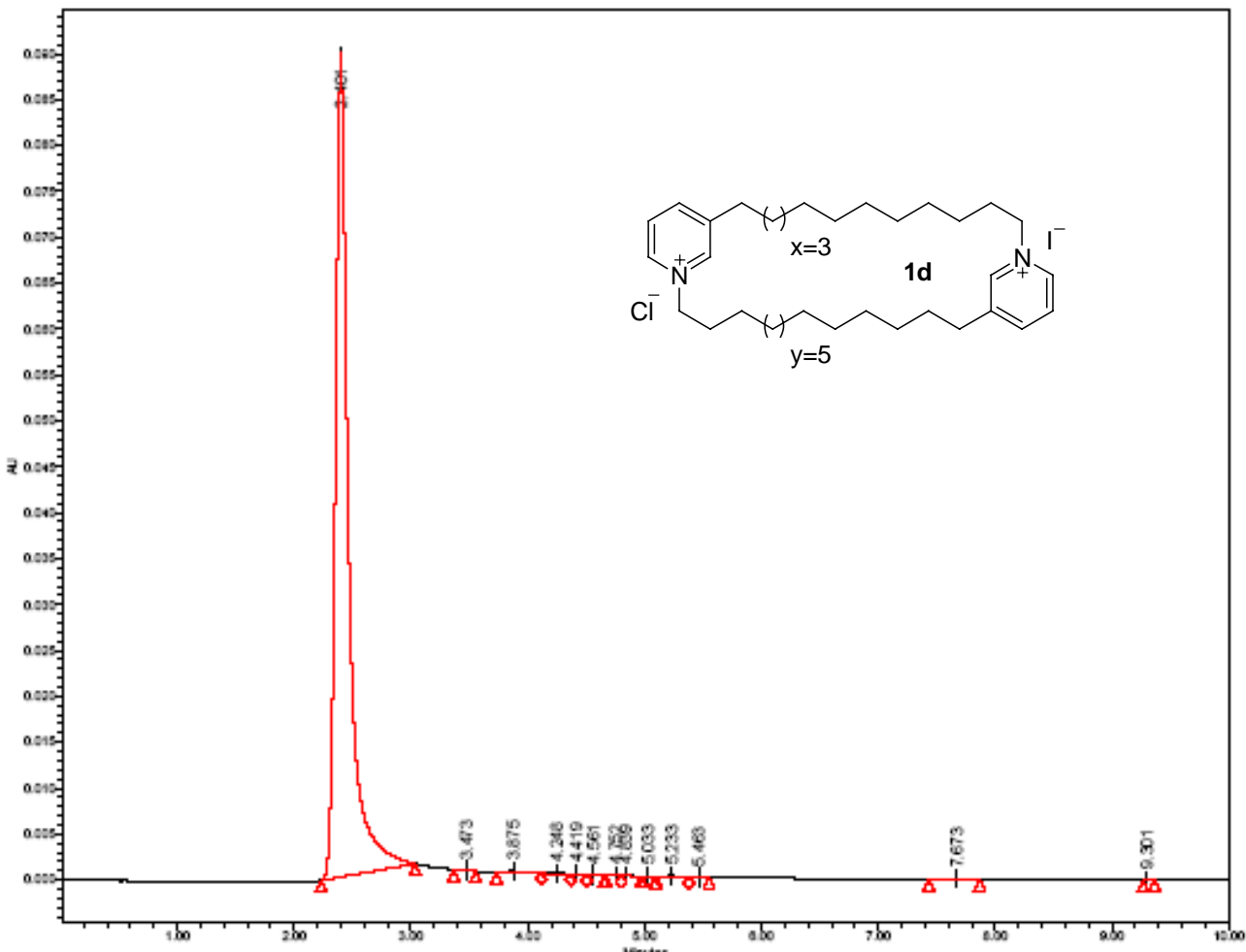

\begin{tabular}{|c|c|c|c|c|c|c|c|c|c|c|}
\hline & Name & $\begin{array}{c}\text { Retention Time } \\
\text { (min) }\end{array}$ & $\begin{array}{c}\text { Area } \\
\left(\mu V^{x} \sec \right)\end{array}$ & $\%$ Area & $\begin{array}{l}\text { Height } \\
(\mu \mathrm{V})\end{array}$ & Int Type & Amount & Units & Peak Type & Peak Codes \\
\hline 1 & & 2.401 & 710589 & 99.27 & 89255 & BB & & & Unknown & \\
\hline 2 & & 3.473 & 89 & 0.01 & 18 & BB & & & Unknown & \\
\hline 3 & & 3.875 & 1716 & 0.24 & 176 & BV & & & Unknown & \\
\hline 4 & & 4.248 & 927 & 0.13 & 107 & W & & & Unknown & \\
\hline 5 & & 4.419 & 258 & 0.04 & 57 & W & & & Unknown & $\mid 19$ \\
\hline 8 & & 4.561 & 175 & 0.02 & 30 & VB & & & Unknown & \\
\hline 7 & & 4.752 & 185 & 0.03 & 35 & BV & & & Unknown & \\
\hline 8 & & 4.839 & 170 & 0.02 & 35 & VB & & & Unknown & 119 \\
\hline 9 & & 5.033 & 33 & 0.00 & 12 & BB & & & Unknown & I19 \\
\hline 10 & & 5.233 & 670 & 0.09 & 90 & BV & & & Unknown & \\
\hline 11 & & 5.483 & 80 & 0.01 & 15 & VB & & & Unknown & \\
\hline 12 & & 7.673 & 885 & 0.12 & 84 & BB & & & Unknown & \\
\hline 13 & & 9.301 & 24 & 0.00 & 8 & BB & & & Unknown & $\mid 19$ \\
\hline
\end{tabular}

Develosil C-30

$\mathrm{H}_{2} 0 / \mathrm{MeOH}: 7 / 93$ (0.1\% TFA)

Flow: $3.2 \mathrm{~mL} / \mathrm{min}$

$\mathrm{v}=267 \mathrm{~nm}$ 

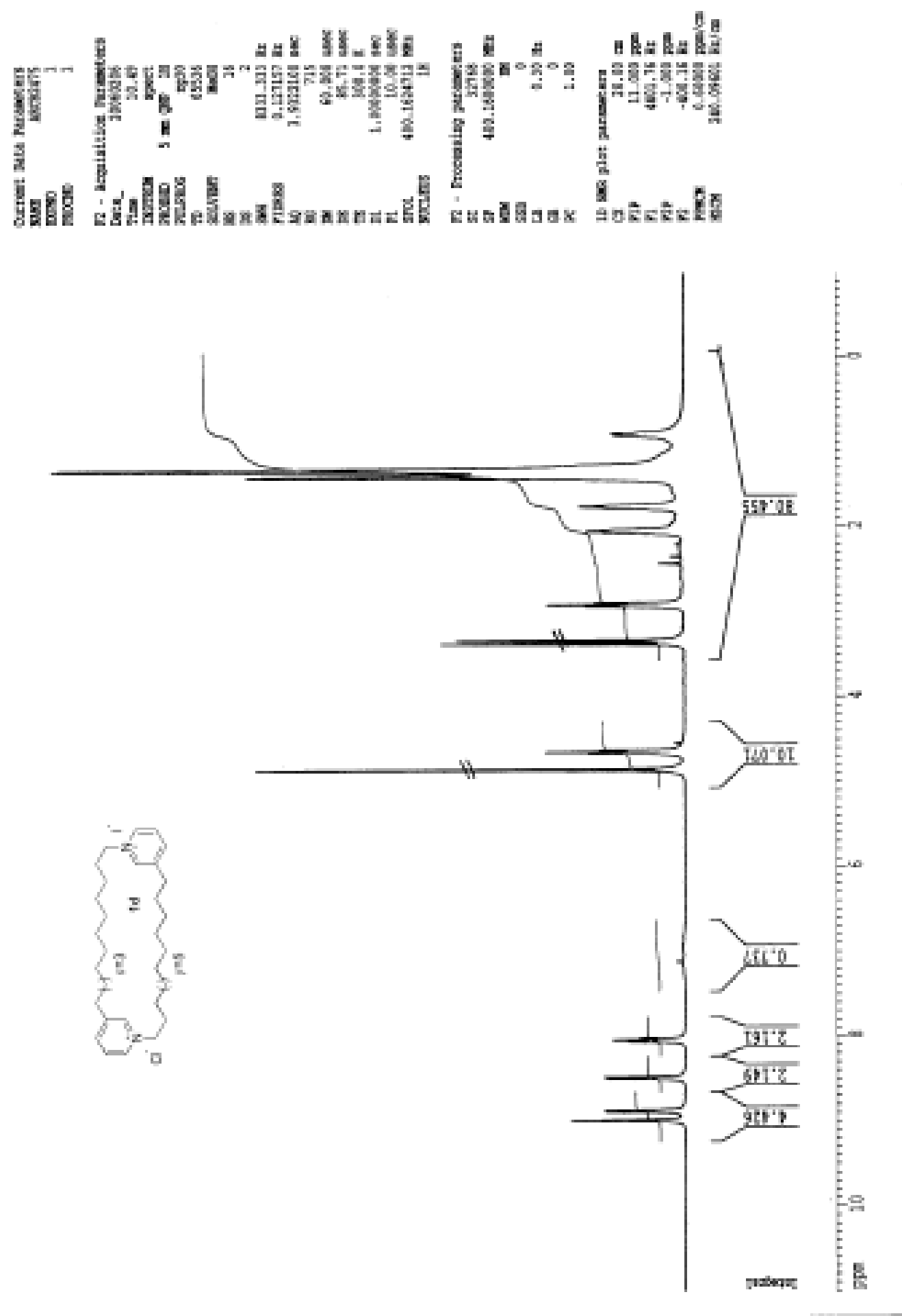

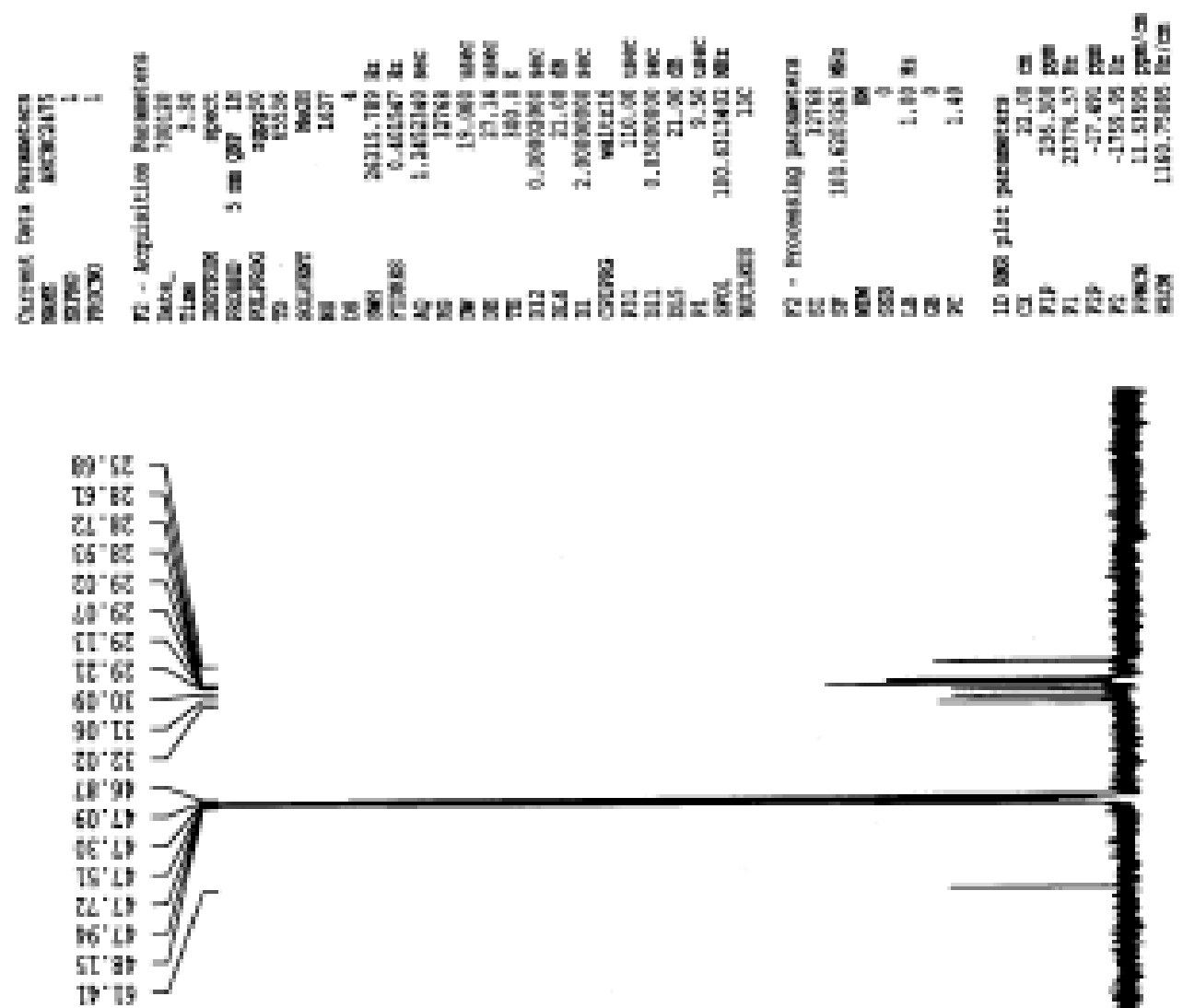

61"

단ㄱ:-

scin-

ten

[T'5).
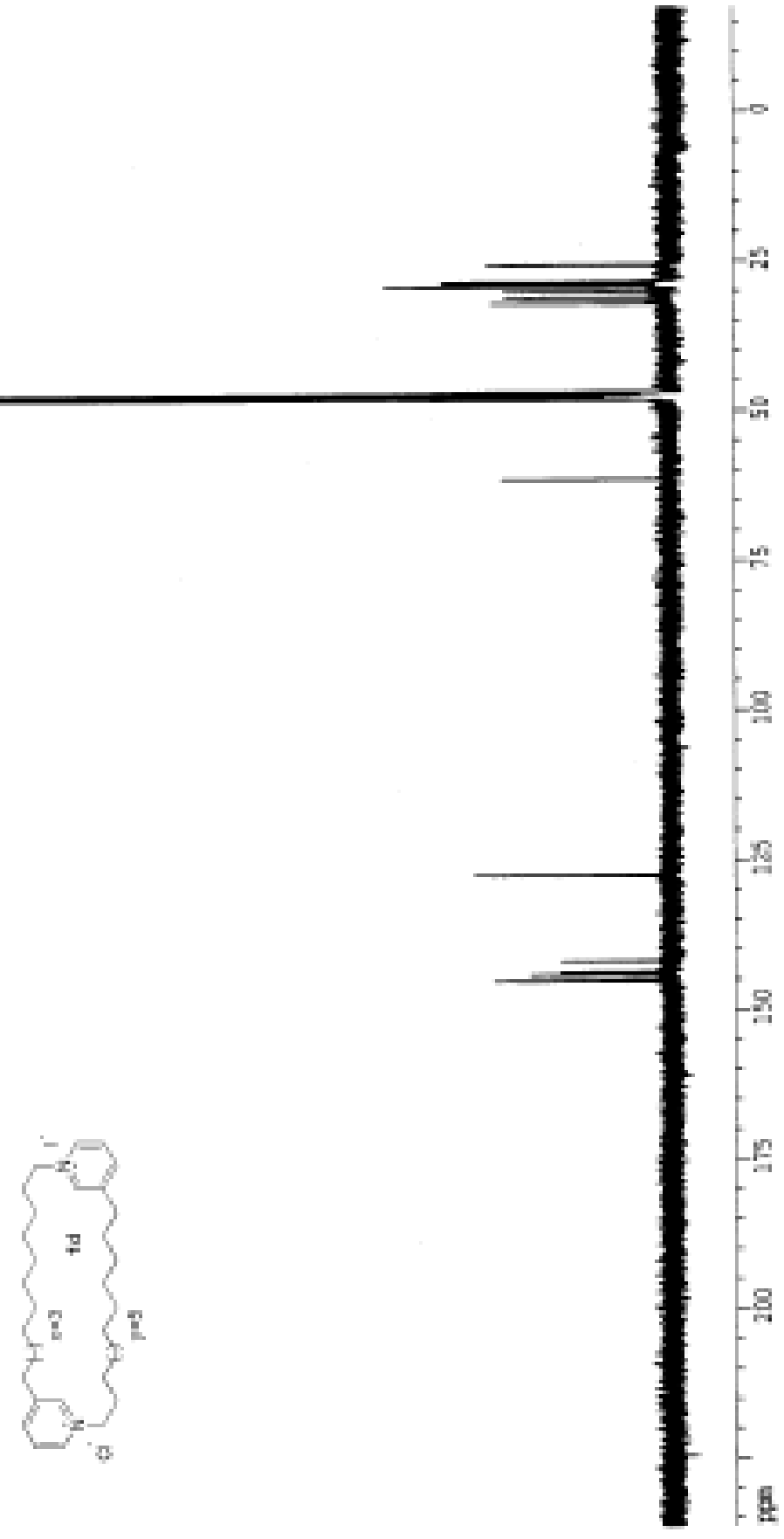

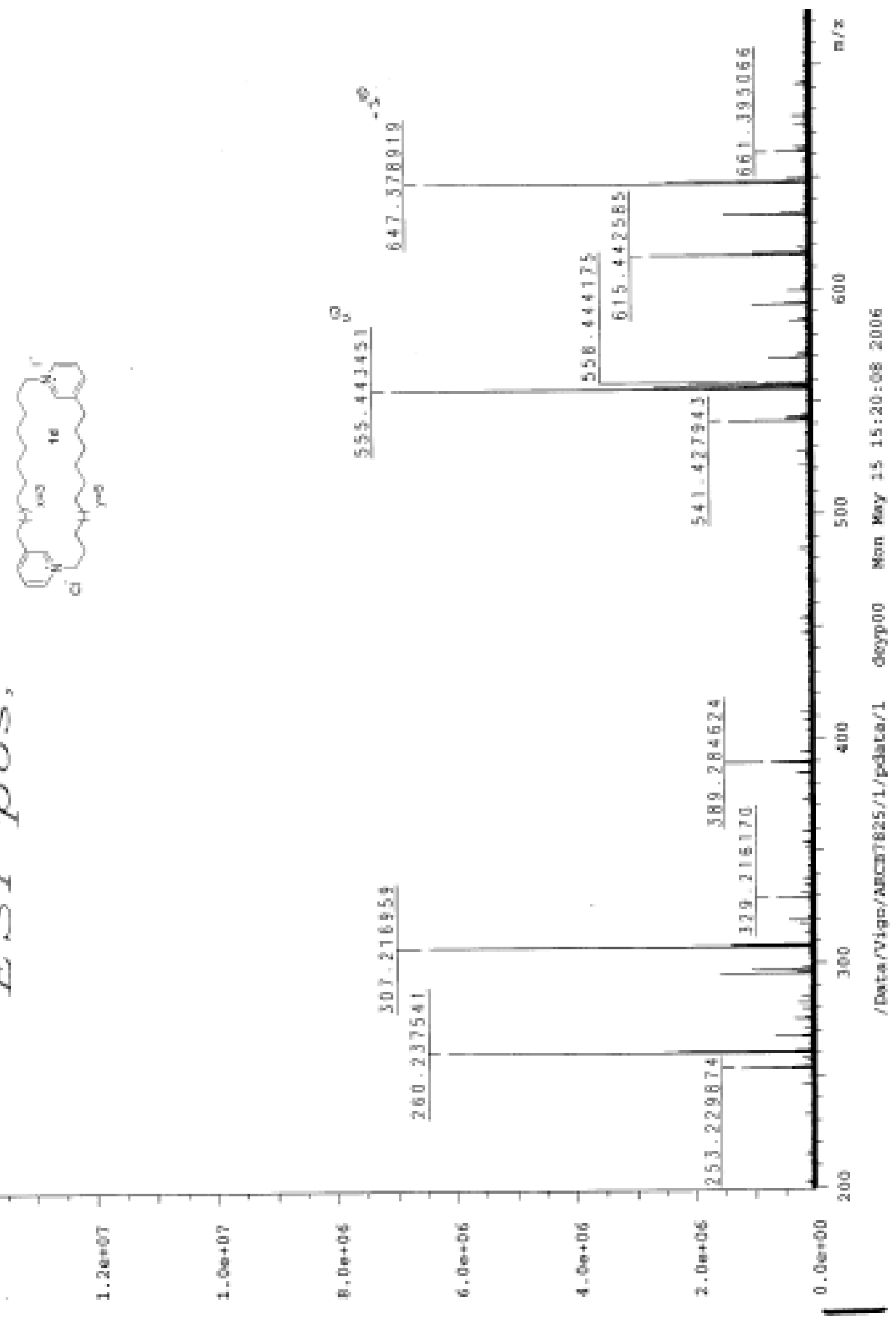


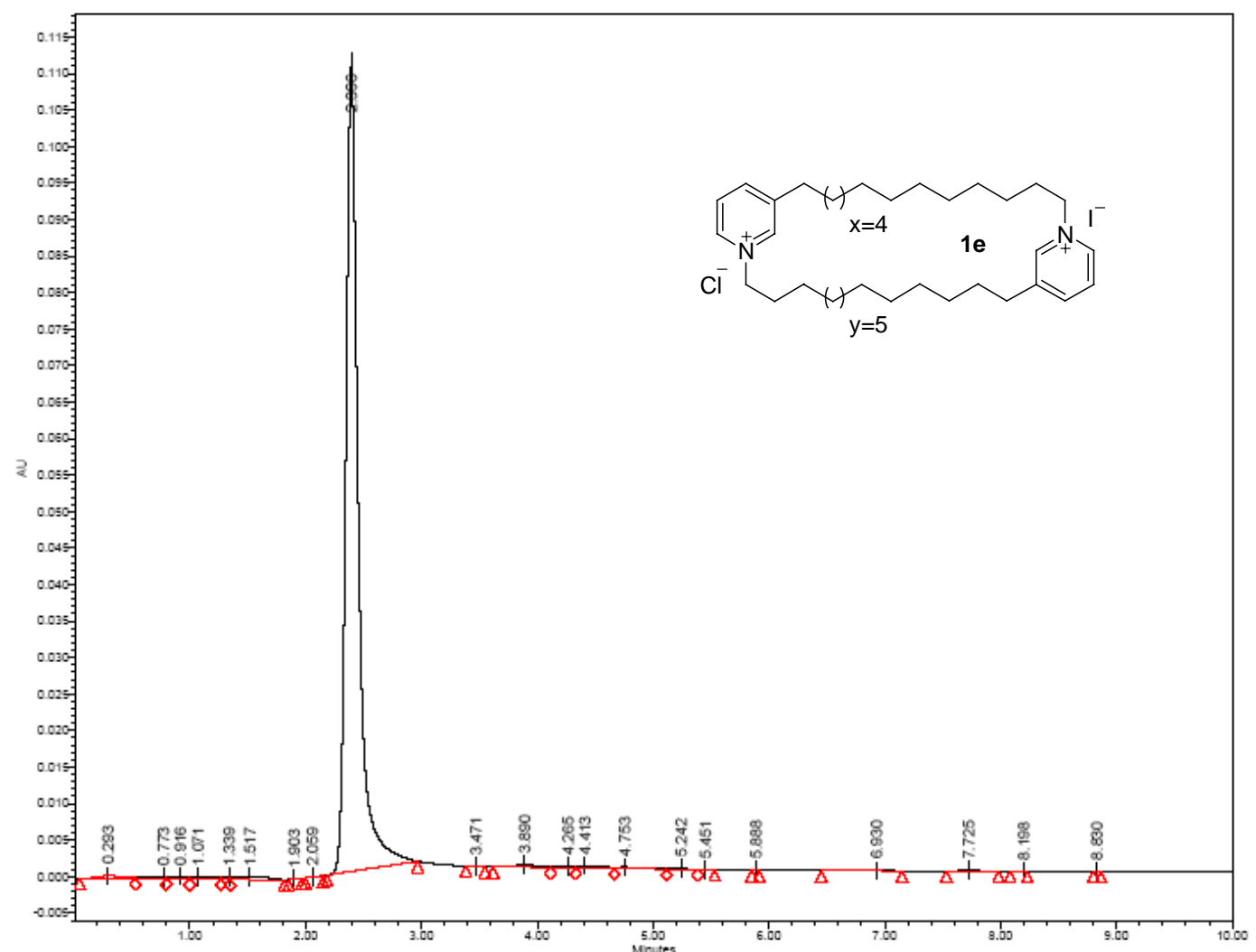

\begin{tabular}{|c|c|c|c|c|c|c|c|c|c|c|}
\hline & Name & \begin{tabular}{|c|}
$\begin{array}{c}\text { Retention Time } \\
(\mathrm{min})\end{array}$ \\
\end{tabular} & $\begin{array}{c}\text { Area } \\
\left(\mu V^{x} \sec \right)\end{array}$ & $\%$ Area & $\begin{array}{l}\text { Height } \\
(\mu \vee)\end{array}$ & Int Type & Amount & Units & Peak Type & Peak Codes \\
\hline 1 & & 0.293 & 6357 & 0.72 & 383 & BV & & & Unknown & \\
\hline 2 & & 0.773 & 4641 & 0.53 & 339 & vV & & & Unknown & \\
\hline 3 & & 0.916 & 4283 & 0.48 & 372 & VV & & & Unknown & \\
\hline 4 & & 1.071 & 5506 & 0.63 & 381 & VV & & & Unknown & \\
\hline 5 & & 1.339 & 1619 & 0.18 & 332 & VV & & & Unknown & 119 \\
\hline 8 & & 1.517 & 7608 & 0.87 & 354 & VB & & & Unknown & \\
\hline 7 & & 1.903 & 202 & 0.02 & 57 & BB & & & Unknown & I19 \\
\hline 8 & & 2.059 & 82 & 0.01 & 14 & $\mathrm{BB}$ & & & Unknown & \\
\hline 9 & & 2.396 & 828709 & 84.48 & 111345 & $B B$ & & & Unknown & \\
\hline 10 & & 3.471 & 128 & 0.01 & 26 & $B B$ & & & Unknown & \\
\hline 11 & & 3.890 & 5093 & 0.58 & 319 & BV & & & Unknown & \\
\hline 12 & & 4.285 & 2959 & 0.34 & 251 & VV & & & Unknown & \\
\hline 13 & & 4.413 & 4145 & 0.47 & 306 & VV & & & Unknown & \\
\hline 14 & & 4.753 & 3034 & 0.35 & 183 & WV & & & Unknown & \\
\hline 15 & & 5.242 & 1050 & 0.12 & 129 & VV & & & Unknown & \\
\hline 16 & & 5.451 & 48 & 0.01 & 10 & VB & & & Unknown & $I 18$ \\
\hline 17 & & 5.888 & 11 & 0.00 & 6 & BB & & & Unknown & 119 \\
\hline 18 & & 6.930 & 759 & 0.09 & 38 & $B B$ & & & Unknown & I19 \\
\hline 19 & & 7.725 & 884 & 0.10 & 83 & $B B$ & & & Unknown & \\
\hline 20 & & 8.198 & 48 & 0.01 & 9 & BB & & & Unknown & 119 \\
\hline 21 & & 8.830 & 12 & 0.00 & 6 & BB & & & Unknown & I19 \\
\hline
\end{tabular}

Develosil C-30

$\mathrm{H}_{2} 0 / \mathrm{MeOH}: 7 / 93$ (0.1\% TFA)

Flow: $3.2 \mathrm{~mL} / \mathrm{min}$

$\mathrm{v}=267 \mathrm{~nm}$ 

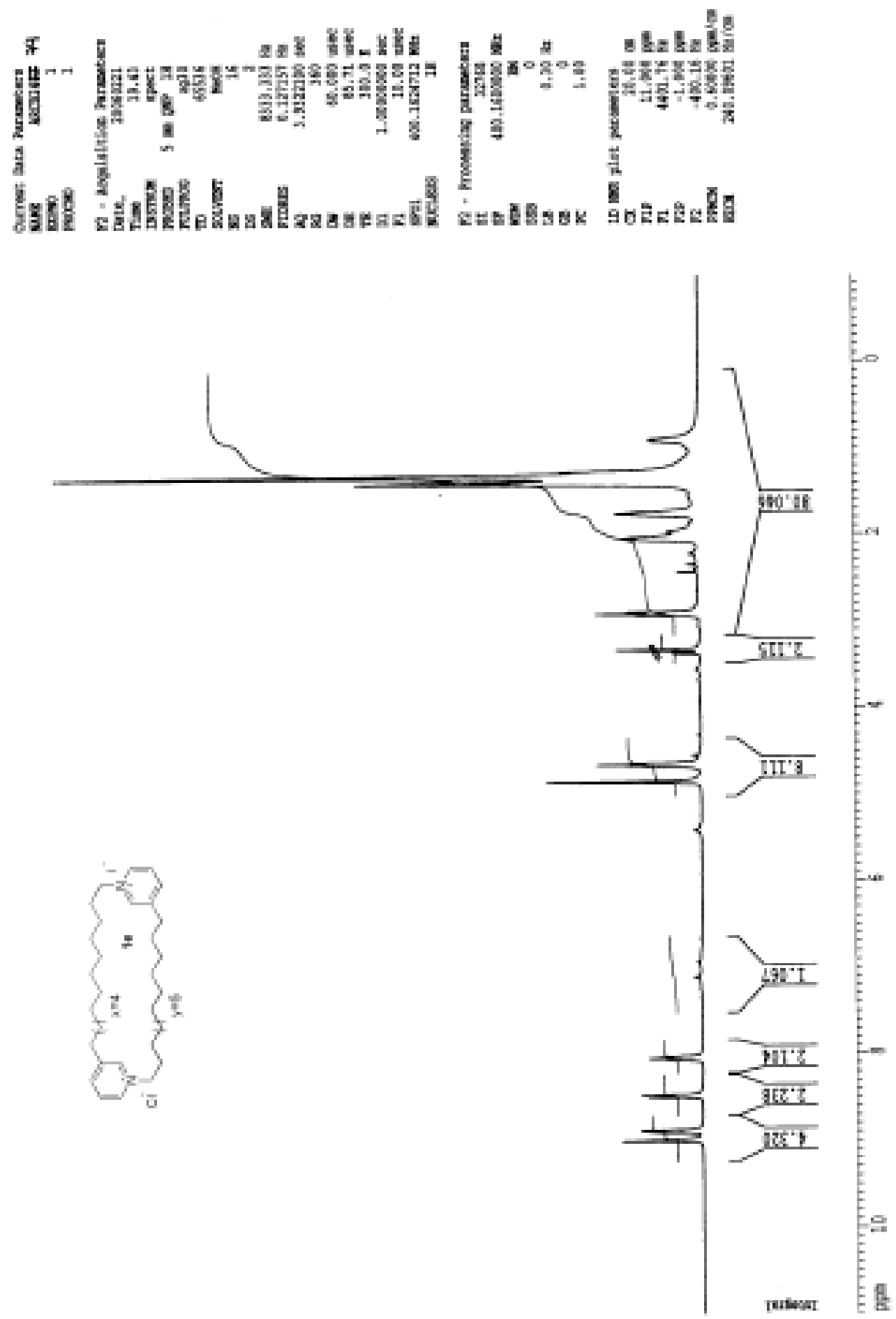


$$
1
$$



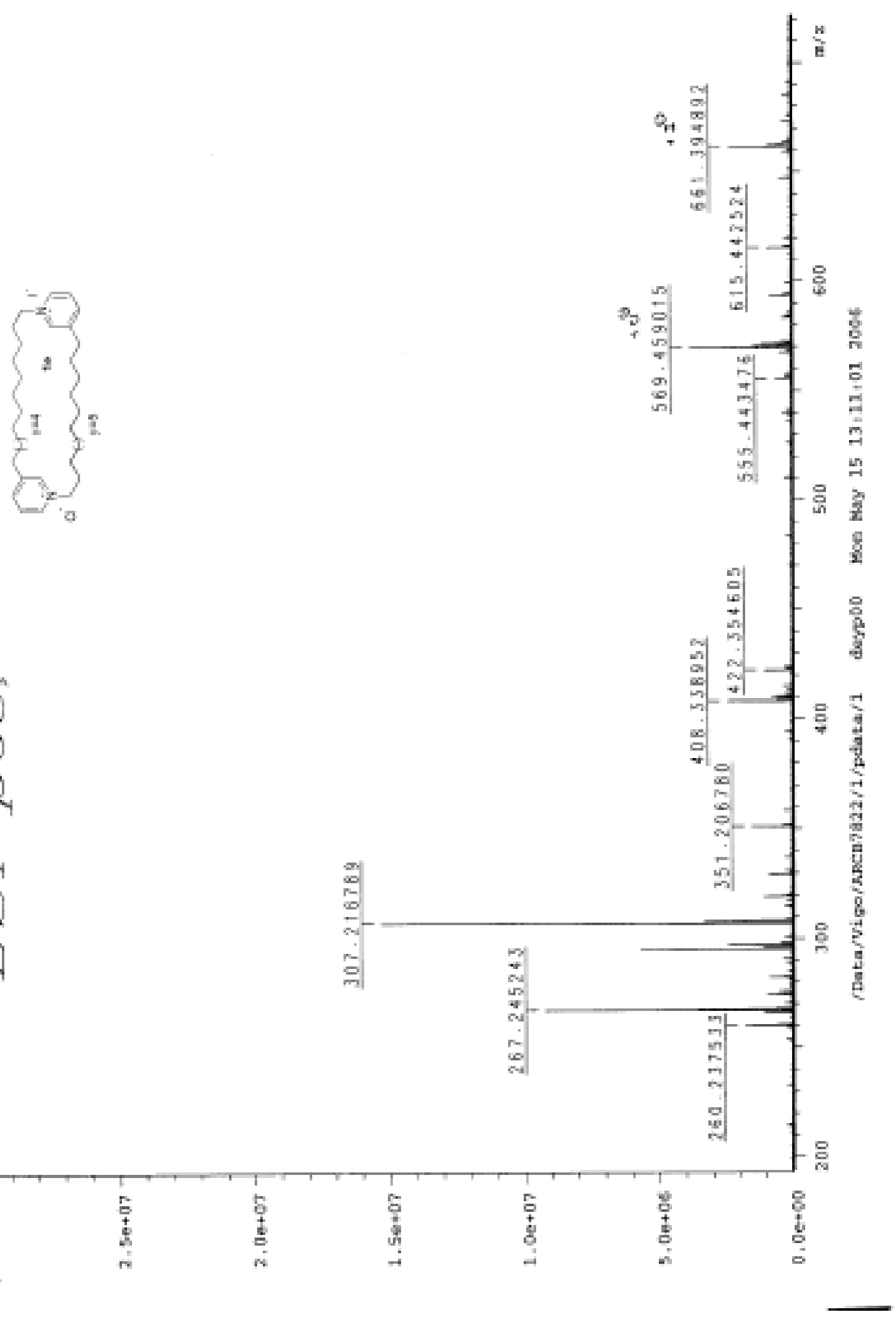


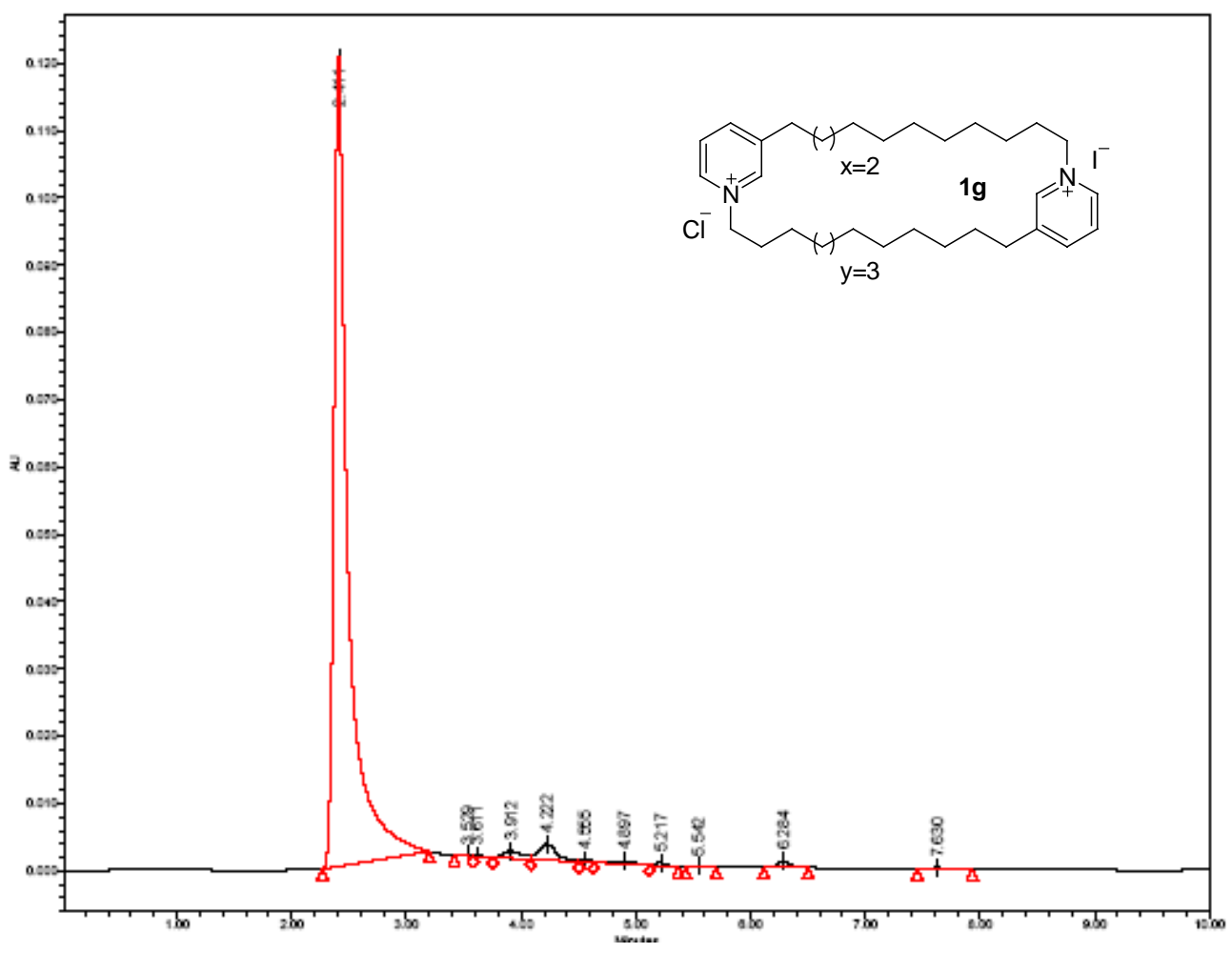

\begin{tabular}{r|r|r|r|r|r|l|r|r|l|l|}
\hline & Name & $\begin{array}{r}\text { Retention Time } \\
(\mathrm{min})\end{array}$ & $\begin{array}{r}\text { Area } \\
\left(\mu \mathrm{V} \mathrm{V}^{\mathrm{sec}}\right)\end{array}$ & $\%$ Area & $\begin{array}{r}\text { Height } \\
(\mu \mathrm{V})\end{array}$ & Int Type & Amount & Units & Peak Type & Peak Codes \\
\hline 1 & & 2.411 & 1054936 & 95.06 & 120471 & $\mathrm{BB}$ & & & Unknown & \\
\hline 2 & & 3.529 & 1187 & 0.11 & 193 & $\mathrm{BV}$ & & & Unknown & \\
\hline 3 & & 3.611 & 1185 & 0.11 & 163 & $\mathrm{~V}$ & & & Unknown & \\
\hline 4 & & 3.912 & 12890 & 1.15 & 1089 & $\mathrm{~W}$ & & & Unknown & \\
\hline 5 & & 4.222 & 26471 & 2.36 & 2472 & $\mathrm{~W}$ & & & Unknown & \\
\hline 6 & & 4.555 & 2016 & 0.18 & 265 & $\mathrm{~W}$ & & & Unknown & \\
\hline 7 & & 4.897 & 8289 & 0.74 & 454 & $\mathrm{~W}$ & & & Unknown & \\
\hline 8 & & 5.217 & 3275 & 0.29 & 472 & VB & & & Unknown & \\
\hline 9 & & 5.542 & 778 & 0.07 & 114 & $\mathrm{BB}$ & & & Unknown & \\
\hline 10 & & 6.284 & 7870 & 0.70 & 1006 & $\mathrm{BB}$ & & & Unknown & \\
\hline 11 & & 7.630 & 2620 & 0.23 & 236 & $\mathrm{BB}$ & & & Unknown & \\
\hline
\end{tabular}

Develosil C-30

$\mathrm{H}_{2} \mathrm{O} / \mathrm{MeOH}: 7 / 93$ (0.1\% TFA)

Flow: $3.2 \mathrm{~mL} / \mathrm{min}$

$\mathrm{v}=267 \mathrm{~nm}$ 
I
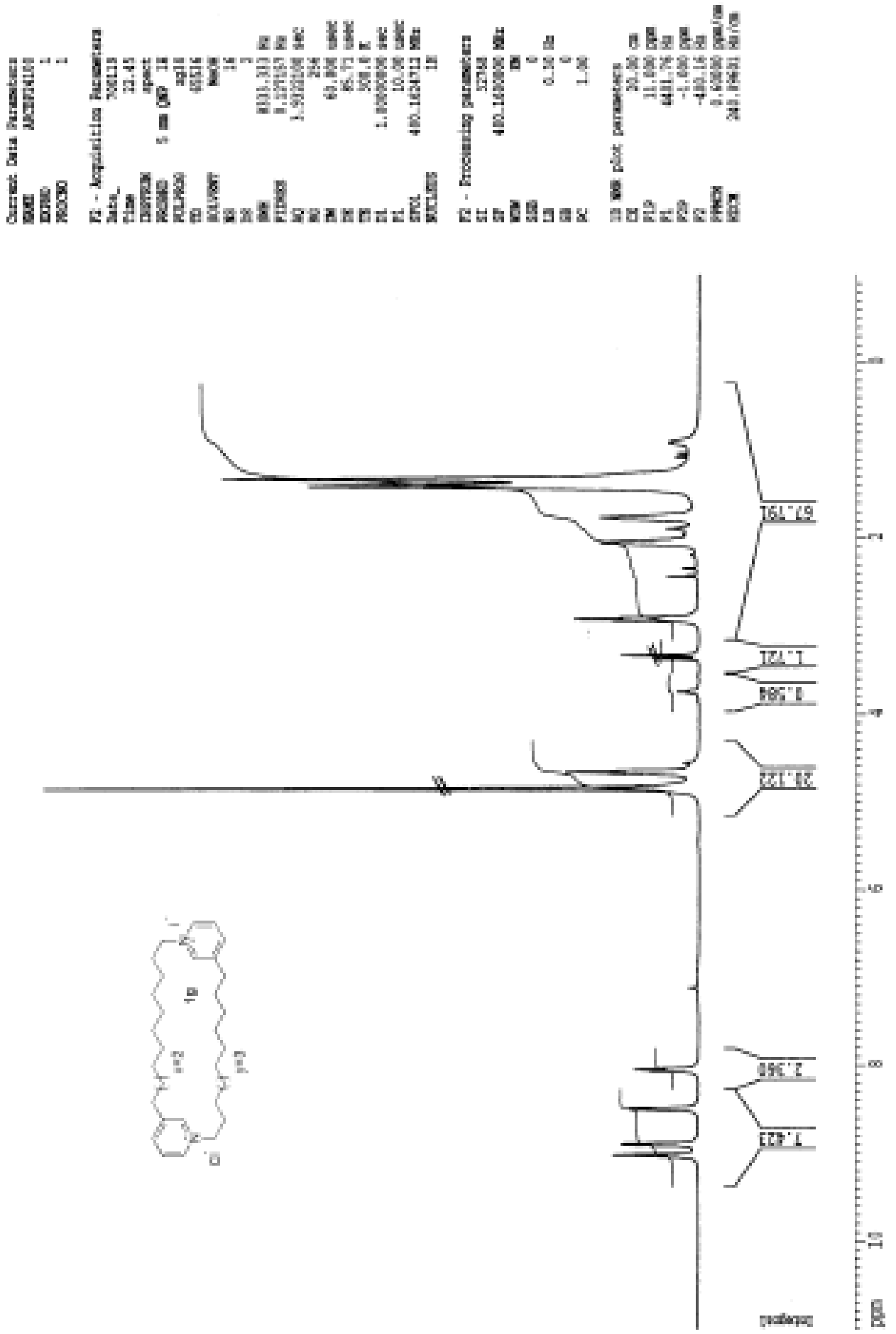

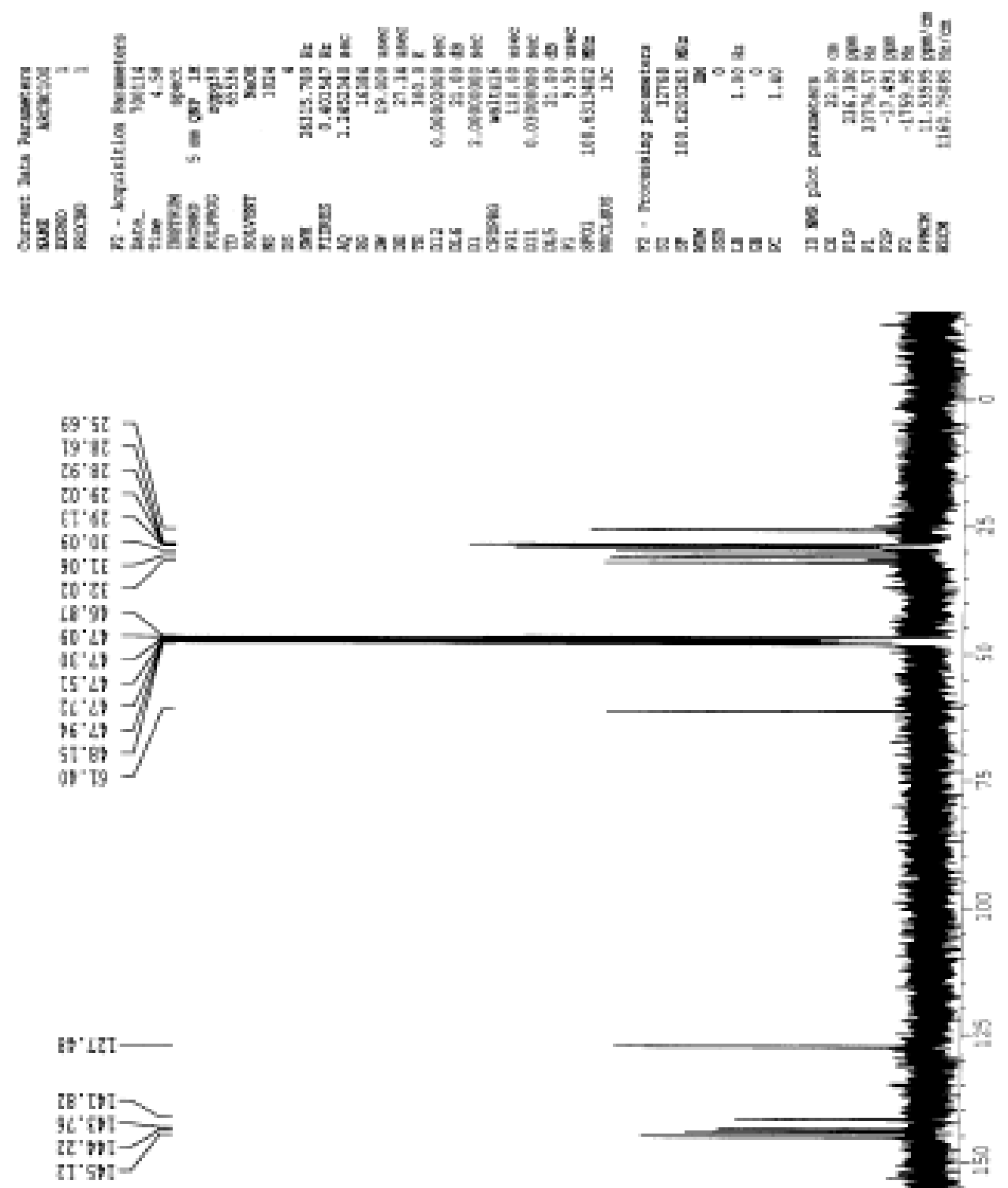


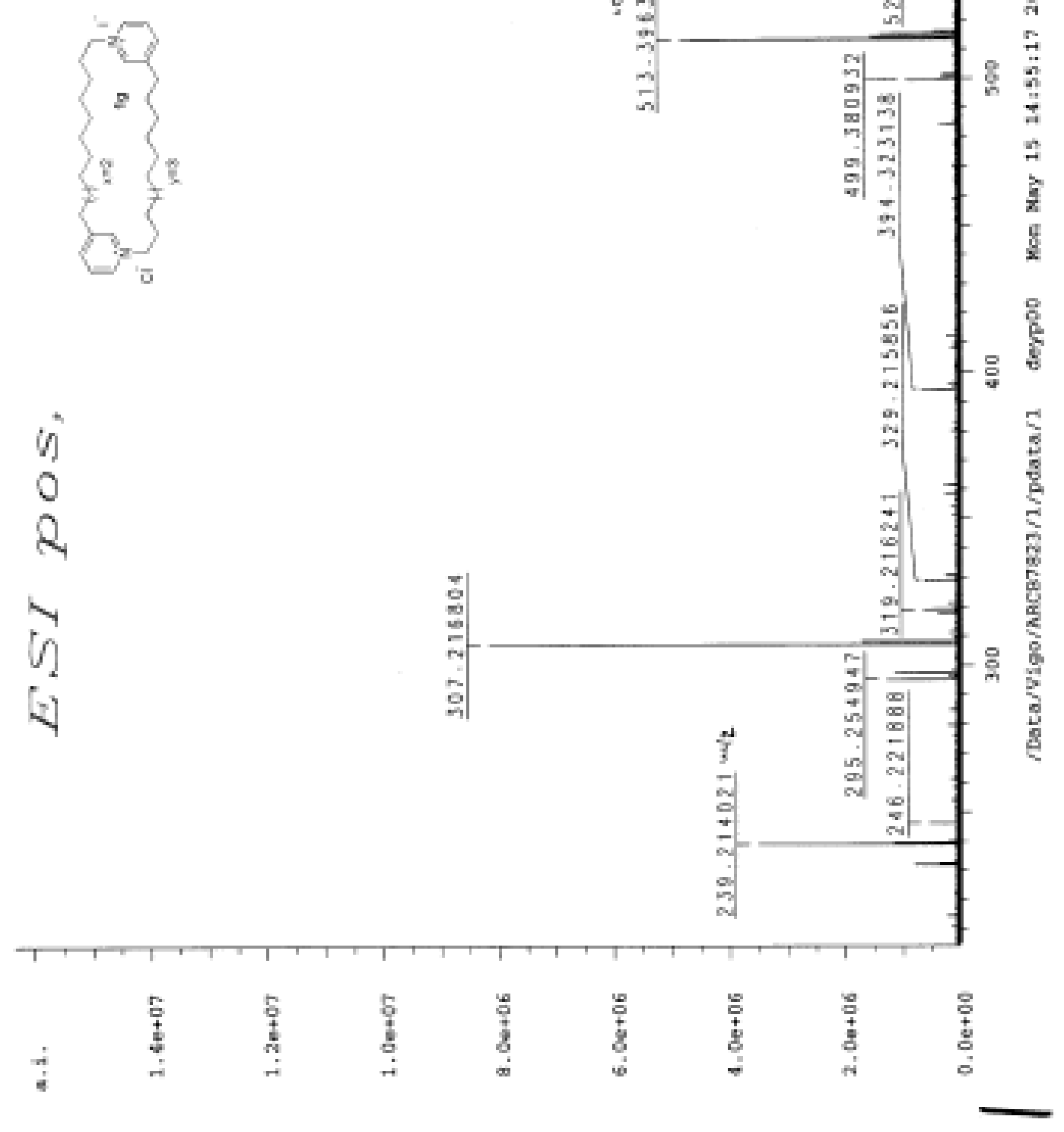




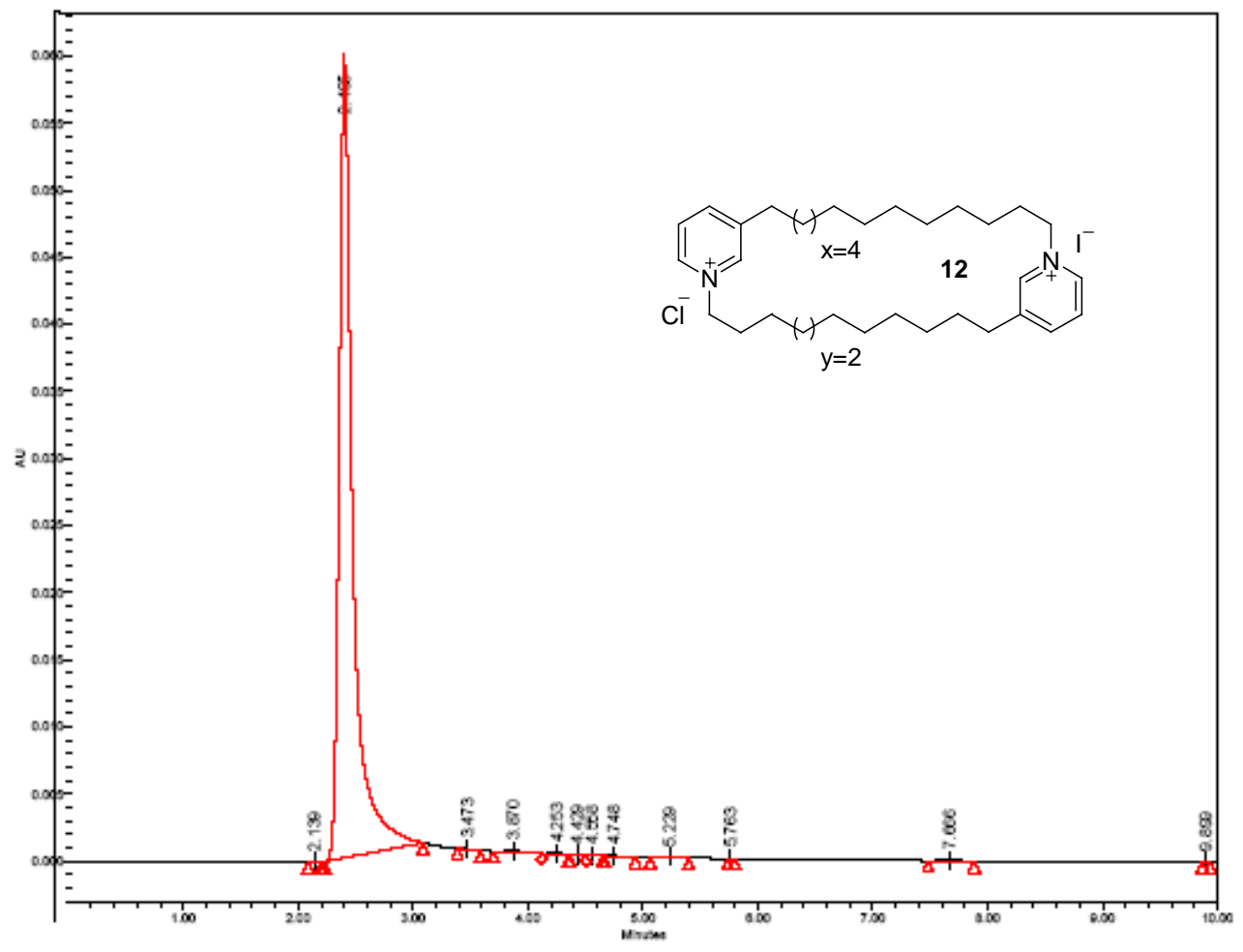

\begin{tabular}{|l|l|r|r|r|r|l|l|l|l|l|}
\hline & Name & $\begin{array}{c}\text { Retention Time } \\
(\mathrm{min})\end{array}$ & $\begin{array}{c}\text { Area } \\
\left(\mu \mathrm{V}^{\prime} \mathrm{sec}\right)\end{array}$ & $\%$ Area & $\begin{array}{c}\text { Height } \\
(\mu \mathrm{V})\end{array}$ & Int Type & Amount & Units & Peak Type & Peak Codes \\
\hline 1 & & 2.139 & 37 & 0.01 & 10 & B B & & & Unknown & I19 \\
\hline 2 & & 2.405 & 499472 & 99.28 & 59774 & B B & & & Unknown & \\
\hline 3 & & 3.473 & 111 & 0.02 & 20 & B B & & & Unknown & \\
\hline 4 & & 3.870 & 1275 & 0.25 & 140 & BV & & & Unknown & \\
\hline 5 & & 4.253 & 644 & 0.13 & 82 & VB & & & Unknown & \\
\hline 6 & & 4.429 & 223 & 0.04 & 43 & BV & & & Unknown & \\
\hline 7 & & 4.558 & 139 & 0.03 & 24 & VB & & & Unknown & \\
\hline 8 & & 4.748 & 220 & 0.04 & 32 & BB & & & Unknown & \\
\hline 9 & & 5.229 & 455 & 0.09 & 58 & BB & & & Unknown & \\
\hline 10 & & 5.783 & 13 & 0.00 & 6 & BB & & & Unknown & I19 \\
\hline 11 & & 7.686 & 616 & 0.12 & 57 & BB & & & Unknown & \\
\hline 12 & & 9.899 & 15 & 0.00 & 7 & BB & & & Unknown & I19 \\
\hline
\end{tabular}

Develosil C-30

$\mathrm{H}_{2} \mathrm{O} / \mathrm{MeOH}: 7 / 93$ (0.1\% TFA)

Flow: $3.2 \mathrm{~mL} / \mathrm{min}$

$\mathrm{v}=267 \mathrm{~nm}$ 

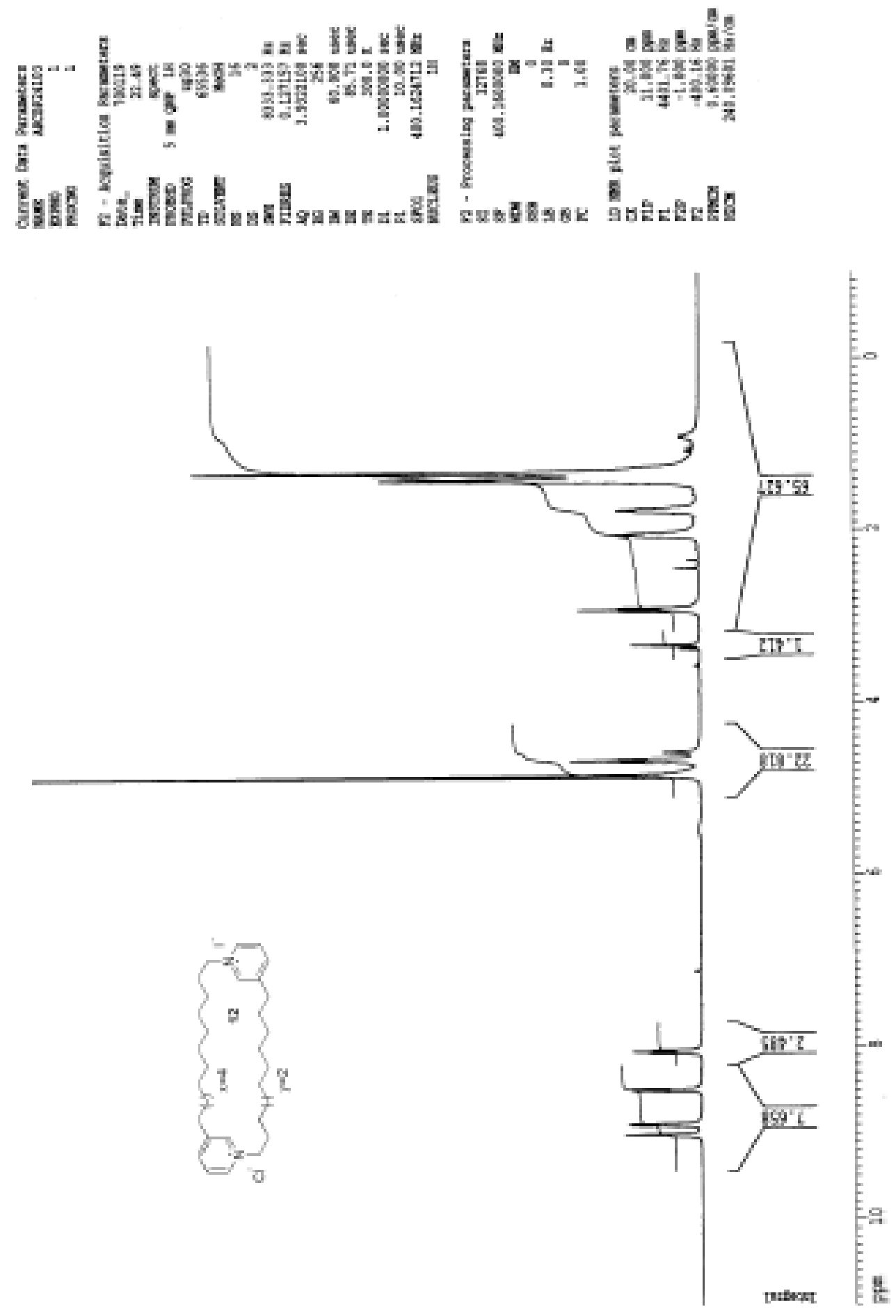

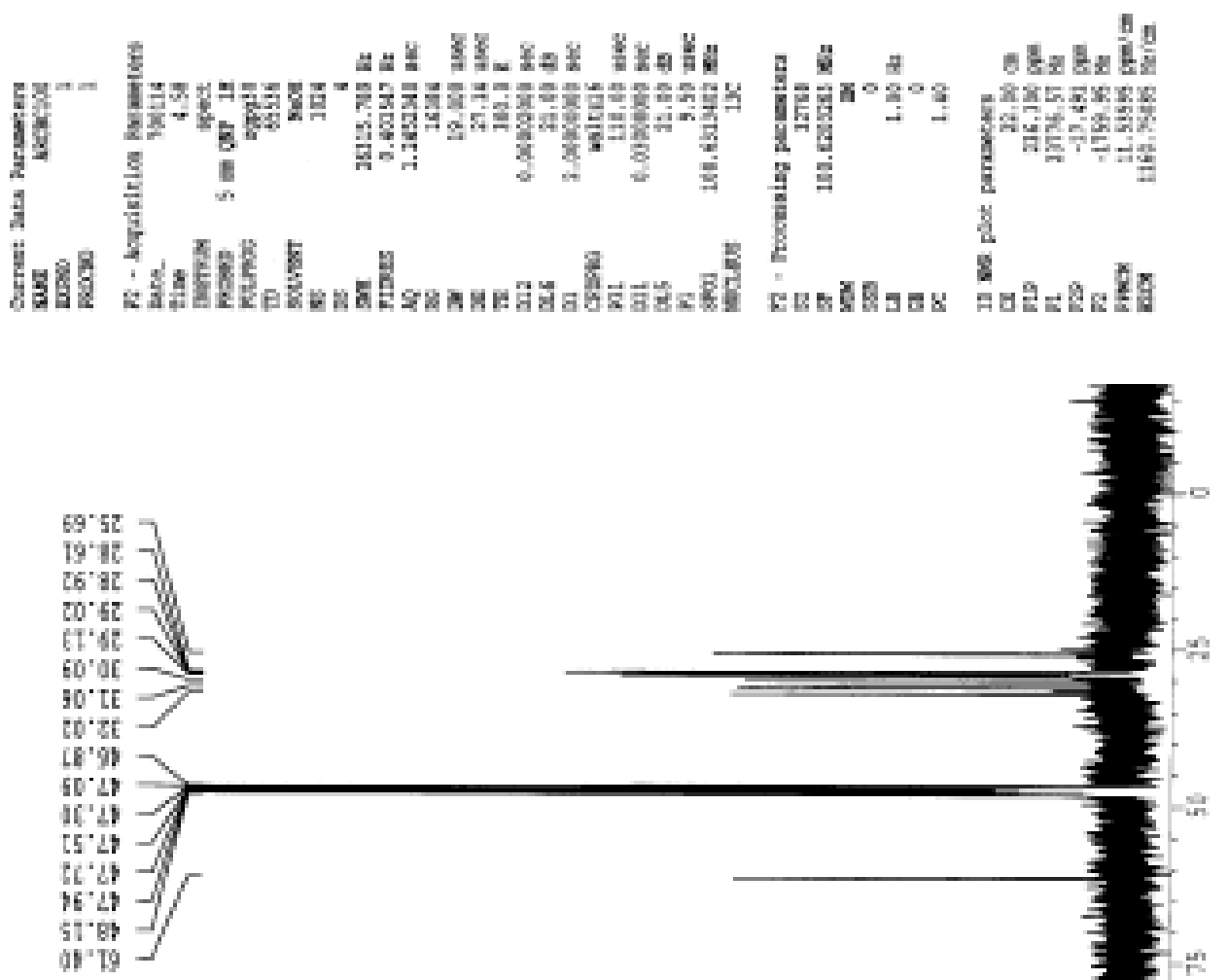

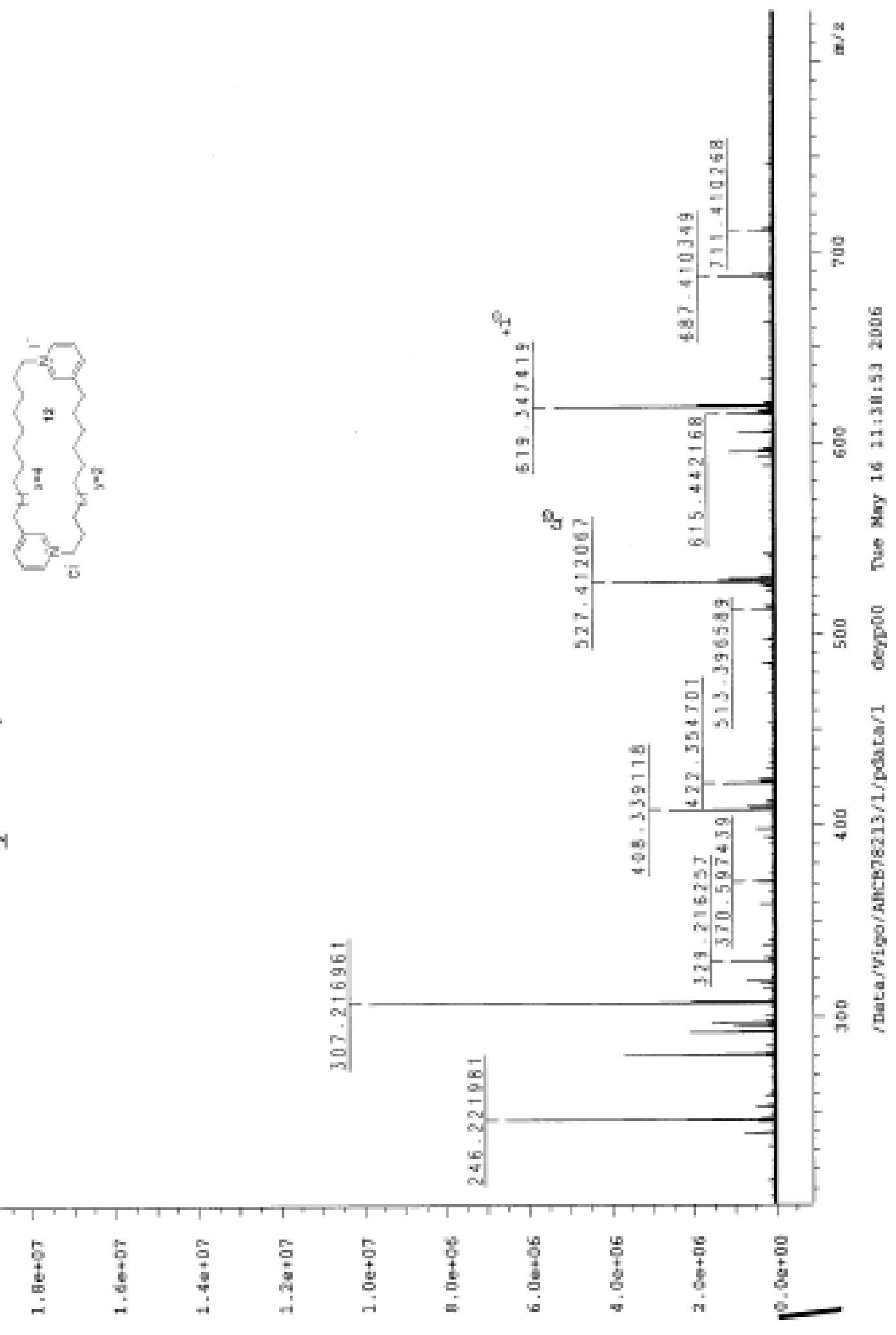


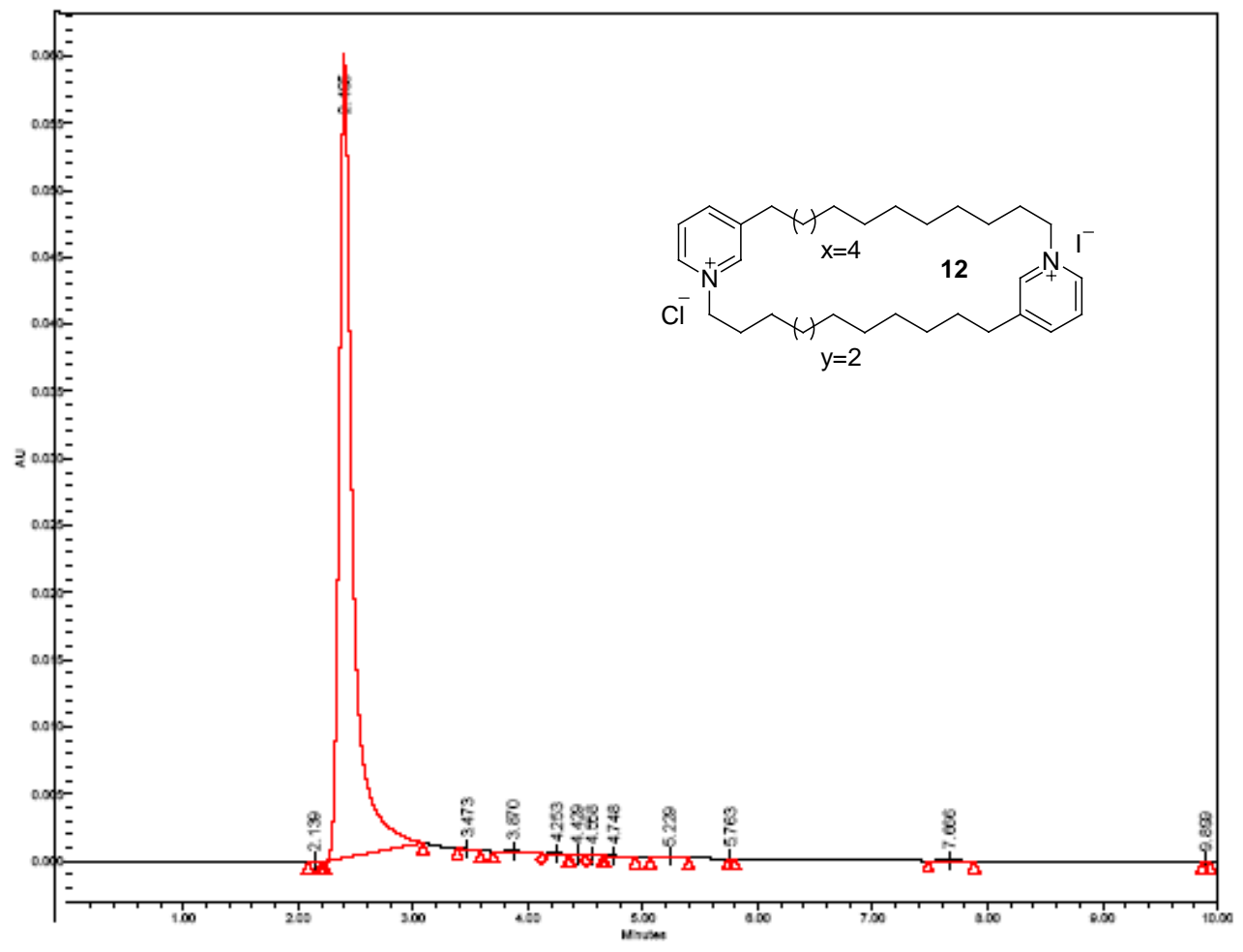

\begin{tabular}{|l|l|r|r|r|r|l|l|l|l|l|}
\hline & Name & $\begin{array}{c}\text { Retention Time } \\
(\mathrm{min})\end{array}$ & $\begin{array}{c}\text { Area } \\
\left(\mu \mathrm{V}^{\prime} \mathrm{sec}\right)\end{array}$ & $\%$ Area & $\begin{array}{c}\text { Height } \\
(\mu \mathrm{V})\end{array}$ & Int Type & Amount & Units & Peak Type & Peak Codes \\
\hline 1 & & 2.139 & 37 & 0.01 & 10 & B B & & & Unknown & I19 \\
\hline 2 & & 2.405 & 499472 & 99.28 & 59774 & B B & & & Unknown & \\
\hline 3 & & 3.473 & 111 & 0.02 & 20 & B B & & & Unknown & \\
\hline 4 & & 3.870 & 1275 & 0.25 & 140 & BV & & & Unknown & \\
\hline 5 & & 4.253 & 644 & 0.13 & 82 & VB & & & Unknown & \\
\hline 6 & & 4.429 & 223 & 0.04 & 43 & BV & & & Unknown & \\
\hline 7 & & 4.558 & 139 & 0.03 & 24 & VB & & & Unknown & \\
\hline 8 & & 4.748 & 220 & 0.04 & 32 & BB & & & Unknown & \\
\hline 9 & & 5.229 & 455 & 0.09 & 58 & BB & & & Unknown & \\
\hline 10 & & 5.783 & 13 & 0.00 & 6 & BB & & & Unknown & I19 \\
\hline 11 & & 7.686 & 616 & 0.12 & 57 & BB & & & Unknown & \\
\hline 12 & & 9.899 & 15 & 0.00 & 7 & BB & & & Unknown & I19 \\
\hline
\end{tabular}

Develosil C-30

$\mathrm{H}_{2} \mathrm{O} / \mathrm{MeOH}: 7 / 93$ (0.1\% TFA)

Flow: $3.2 \mathrm{~mL} / \mathrm{min}$

$\mathrm{v}=267 \mathrm{~nm}$ 

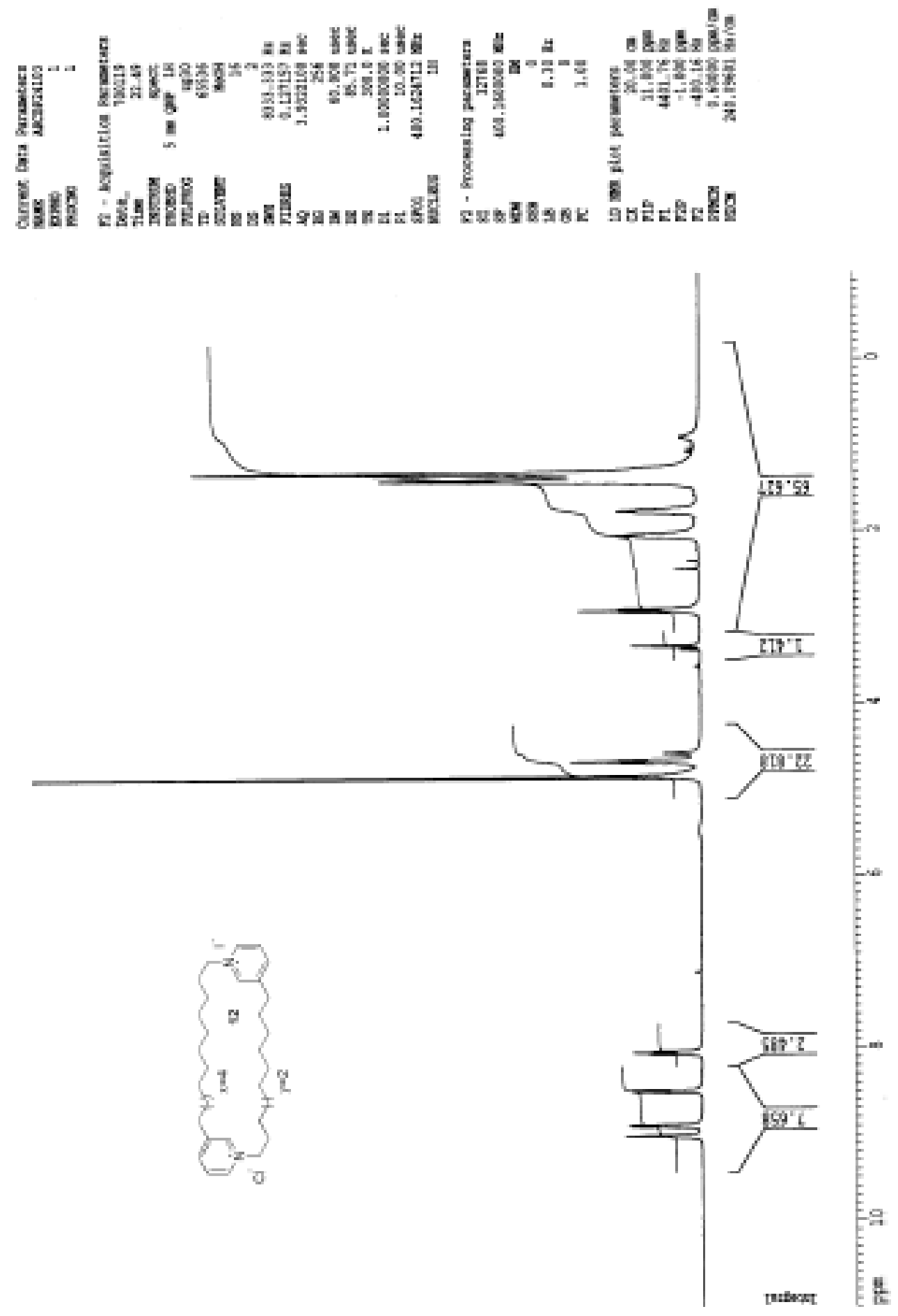

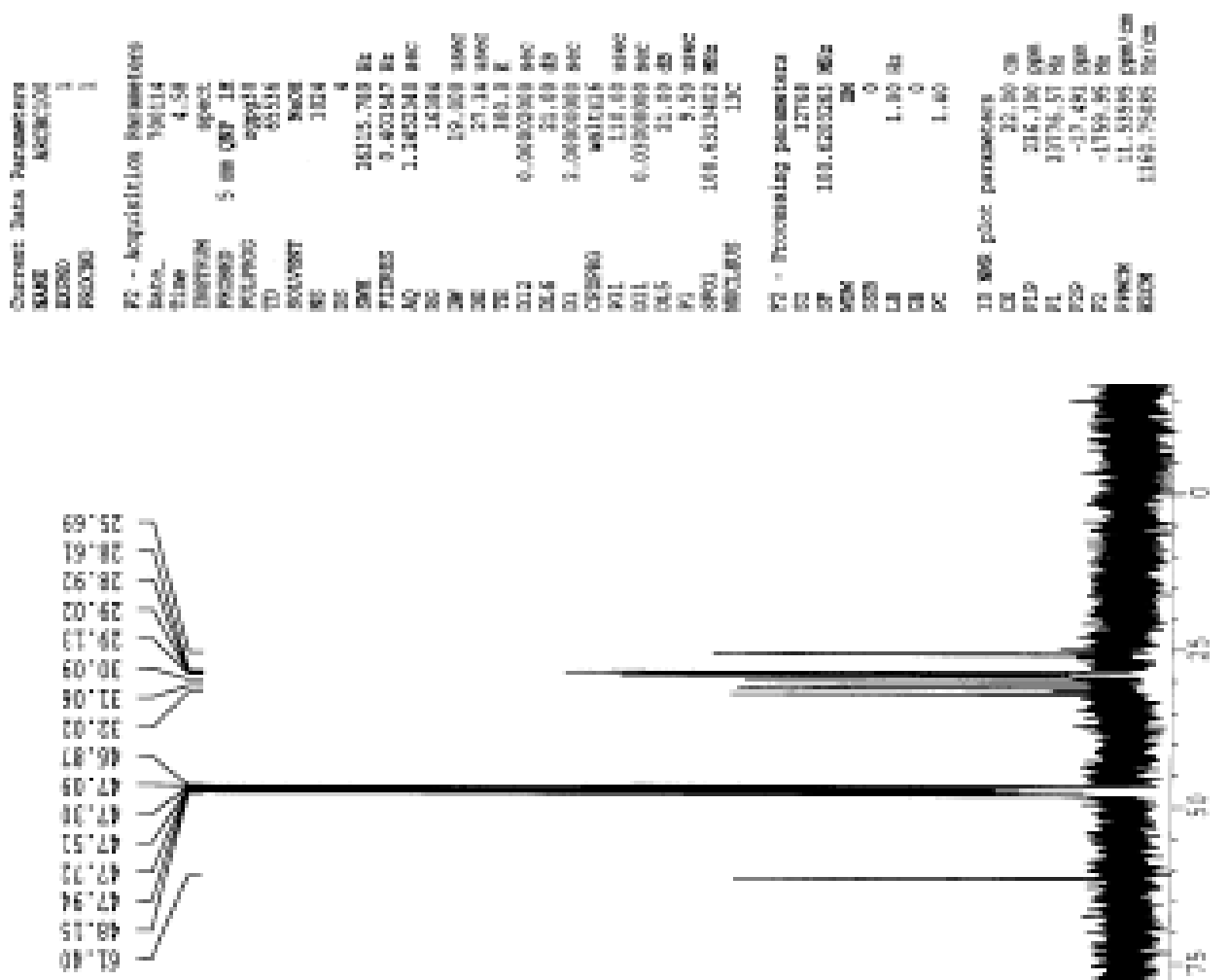

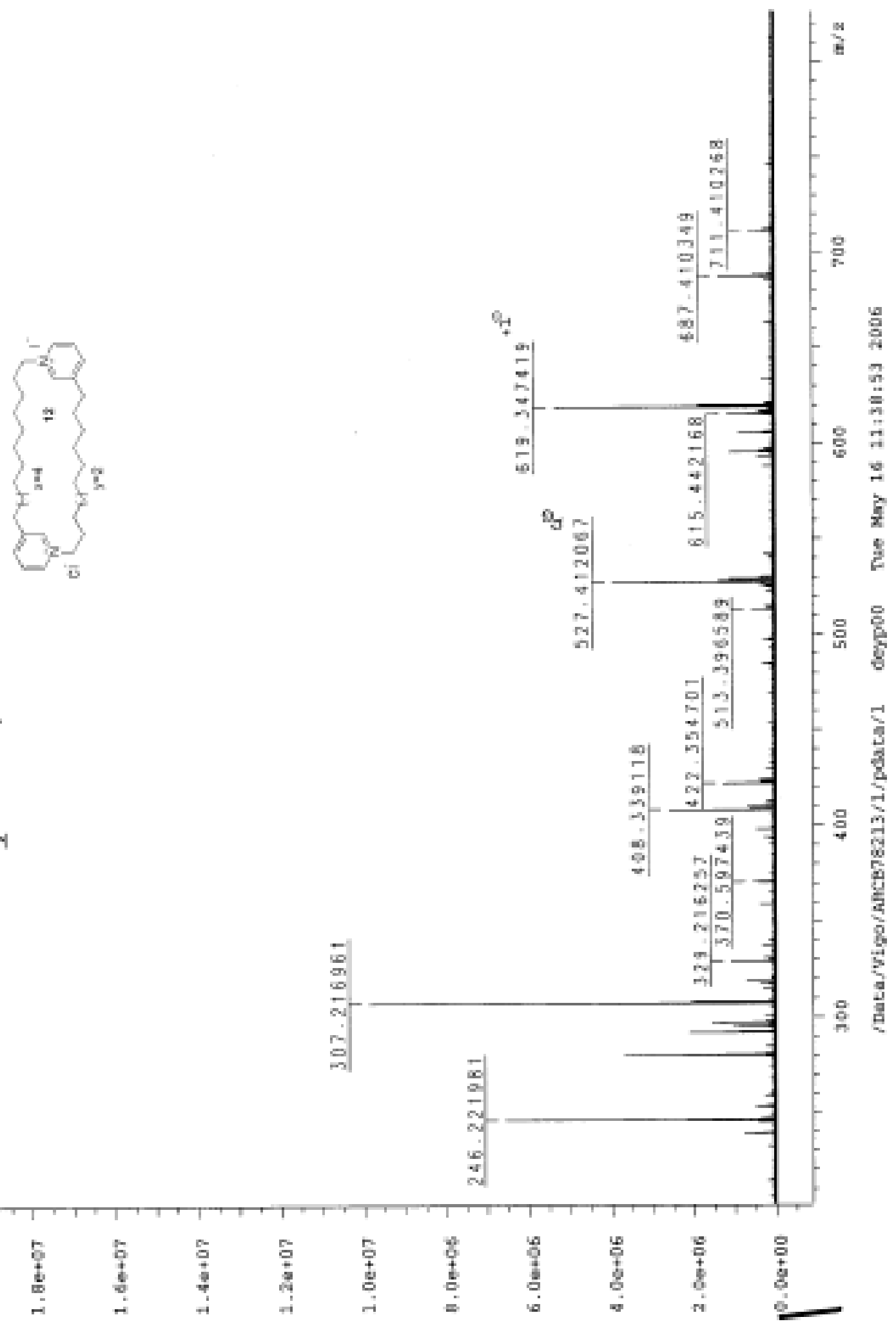